\title{
Atom Efficient Synthesis of Selectively Difluorinated Carbocycles through a Gold(I) Catalyzed Cyclization
}

Adam W. McCarter, ${ }^{\dagger}$ Magdalena Sommer,${ }^{\dagger}$ Jonathan M. Percy, ${ }^{* \dagger}$ Craig Jamieson, ${ }^{* \dagger}$ Alan R. Kennedy, ${ }^{\dagger}$ and David J. Hirst

$\dagger$ Department of Pure and Applied Chemistry, University of Strathclyde, 295 Cathedral Street, Glasgow G1 1XL, UK.

$¥$ GlaxoSmithKline R\&D Ltd., Gunnels Wood Road, Stevenage, Herts SG1 2NY, UK.

*Email: craig.jamieson@strath.ac.uk; jonathan.m.percy@btinternet.com

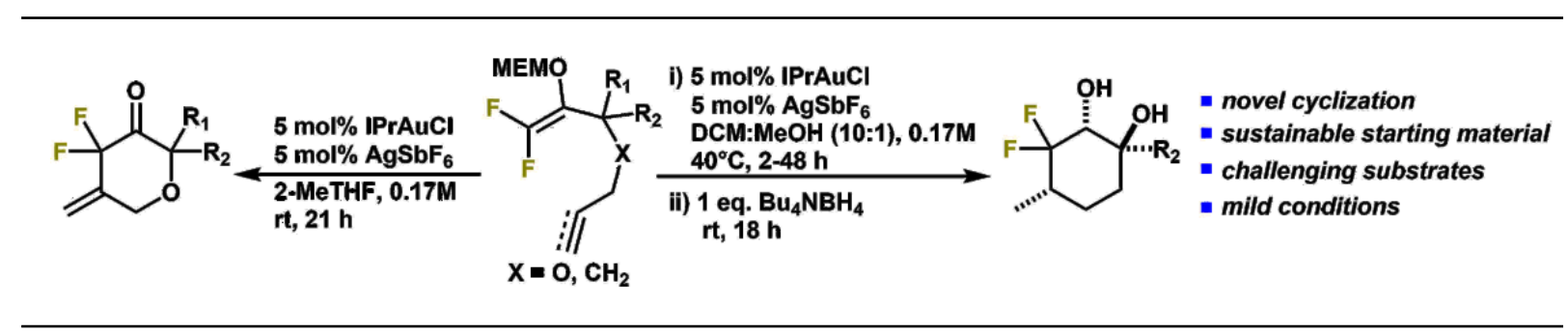

\section{Abstract}

The intramolecular carbocyclization of difluorinated enol acetals has been achieved for the first time using gold(I) catalysis. Difluorinated enol acetals bearing a pendant alkene group can be cyclized and reduced in one pot to form fluorinated diol motifs. Alternatively, the cyclization of terminal alkynes allows for the synthesis of fluorinated pyran scaffolds. Both cyclization processes can be performed under mild conditions allowing access to complex products rich in functionality. The cyclic systems are synthesized concisely (maximum four steps) from trifluoroethanol, an inexpensive fluorinated feedstock.

\section{Introduction}

The design and synthesis of geminal difluoro compounds is an extremely important objective in medicinal chemistry owing to their unique biological properties, such as enzyme 
inhibition, pKa modulation and improving metabolic stability. ${ }^{1}$ However, efficient methods, starting from sustainable low cost starting materials, for the introduction of the gemdifluoromethylene group into cyclic molecules are still scarce. One of the most commonly implemented methods involves fluorination of a cyclic ketone with a nucleophilic fluorinating agent such as diethylaminosulfur trifluoride (DAST). ${ }^{1 \mathrm{~b}}$ An alternative method recently reported independently by the groups of Amii and Dilman utilizes a difluorocyclopropanation/ring expansion strategy to effectively introduce the $-\mathrm{CF}_{2}$ moiety (Scheme 1). ${ }^{2,3}$

- Diederich<smiles>CC(=O)O[C@H]1CC2(CCC1=O)OCCO2</smiles>

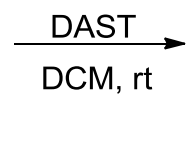<smiles>CC(=O)O[C@H]1CC2(CCC1(F)F)OCCO2</smiles>

- Amii<smiles>COC1=CCCCC1</smiles>

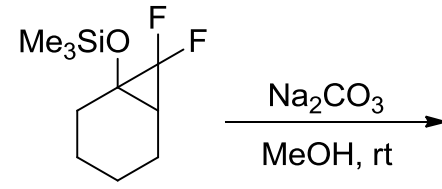

$73 \%$<smiles>O=C1CCCCCC1(F)F</smiles>

$71 \%$

Scheme 1. Selection of methods for preparing difluorinated carbocycles.

Annulation chemistry based on difluorinated building blocks is much less well established. ${ }^{4}$ In our efforts towards the development of new methods for the synthesis of selectively difluorinated carbocycles we recently reported the Saegusa-Ito cyclization as a concise and efficient protocol for the construction of selectively difluorinated cyclohexenones (Scheme 2). ${ }^{5}$ 
- Previous work ${ }^{5}$

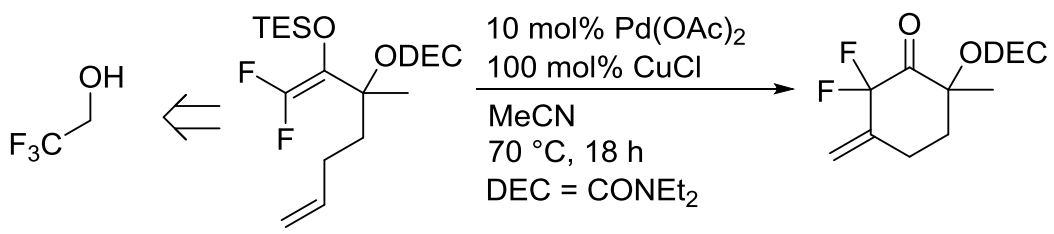

Current study

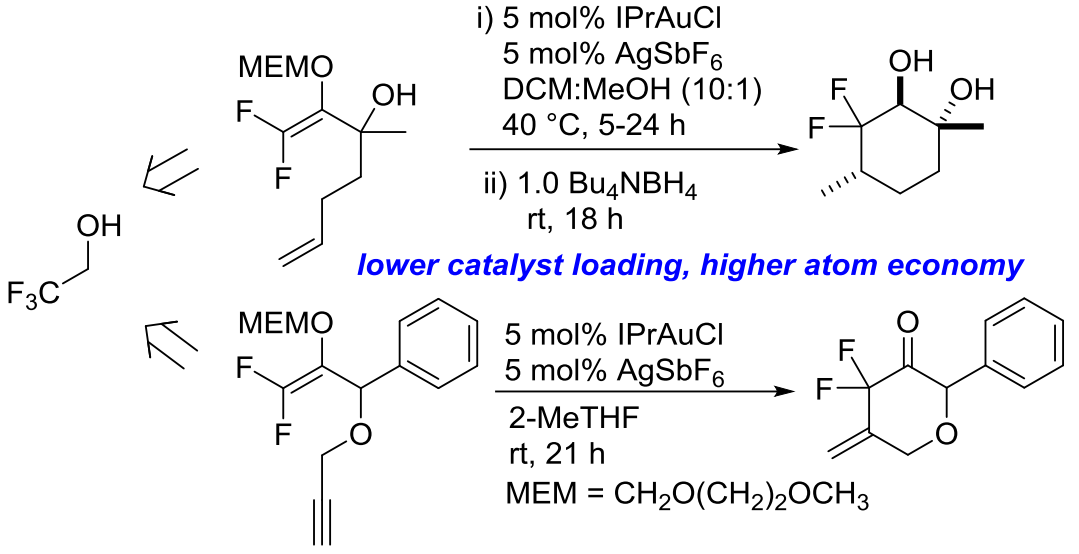

Scheme 2. Context of the current study.

Whilst this method represented a novel and exciting synthetic development, it is limited by poor atom economy arising from the use of two protecting groups, including the very robust carbamate. A more atom efficient cyclization based on difluorinated enol acetal nucleophiles would therefore be an attractive approach. While such species have proved effective substrates in sigmatropic rearrangements, their reactivity as nucleophiles has not been exploited in synthesis. ${ }^{6}$ We envisioned that these species could be utilized in a divergent synthetic strategy to afford either difluorinated diols or pyrans through a gold(I) catalyzed cyclization (Scheme 2). ${ }^{7}$

Results and Discussion

Investigation into alkene cyclization. Following protection of trifluoroethanol, the requisite enol acetal species could be accessed from acetal $\mathbf{1}$ using our previously established one-pot dehydrofluorination/metalation procedure (Scheme 3). ${ }^{8}$ 


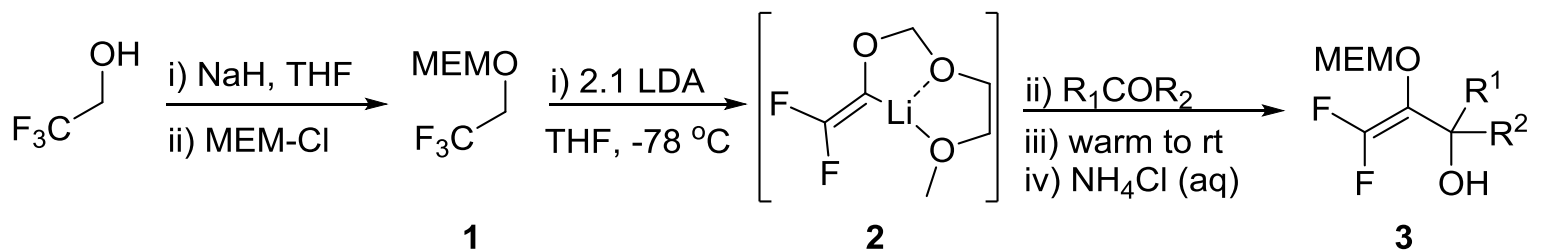
$-\mathrm{OMEM}=-\mathrm{OCH}_{2} \mathrm{O}\left(\mathrm{CH}_{2}\right)_{2} \mathrm{OCH}_{3}$

Scheme 3. Allylic alcohol synthesis

Treatment of $\mathbf{1}$ with lithium diisopropylamide generates the stabilized organolithium intermediate 2 . Addition of a $\gamma, \delta$-unsaturated aldehyde or ketone followed by quenching with aqueous ammonium chloride delivers the desired allylic alcohols 3. This procedure was typically performed on a $>10 \mathrm{mmol}$ scale and, where possible, the products were purified by Kugelrohr distillation. A range of allylic alcohols were synthesized including, heterocyclic, geminally disubstituted and alicyclic analogues (Table 1). 
Table 1: Difluorinated allylic alcohols 3 prepared from 1.
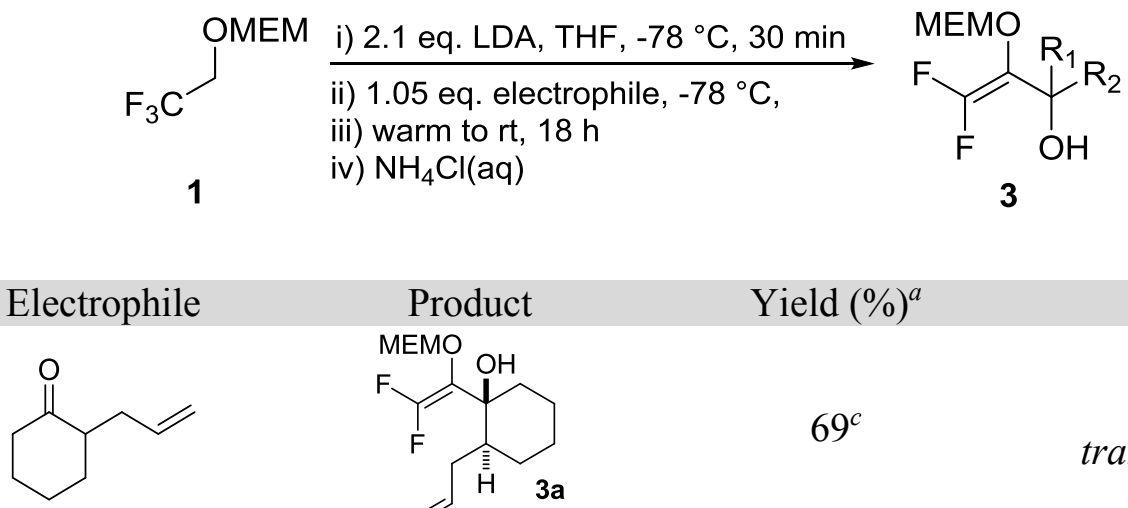

Yield $(\%)^{a}$

$\mathrm{dr}^{b}$<smiles>C=CCC1CCCCC1=O</smiles><smiles>C=CCC1[C+]CCCC1(O)C(OC)(OC)C(F)F</smiles>

$69^{c}$

19:1<smiles>C=CCC1COCCC1=O</smiles>

MEMO<smiles>C=CC[C@H]1COCCC1(O)C(OC)=C(F)F</smiles>

$32: 1$

$32^{c, d}$ trans $\mathbf{- 3 b}$<smiles>C=CCC1CNCCC1=O</smiles><smiles>C=CCC1CN(C(=O)O)CCC1(O)C(O)=C(F)F</smiles>
trans-3c/cis-3c<smiles>C=CCCC(C)=O</smiles>
MEMO<smiles>C=CCCC(C)(O)C(C)=C(F)F</smiles>

MEMO

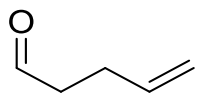<smiles>C=CCCC(O)C(C)=C(F)F</smiles>

MEMO<smiles>C=CC(C)(C)CC=O</smiles><smiles>C=CCC(C)(C)C(O)C(OC)=C(F)F</smiles><smiles>C=CC1(CC(C)=O)CC1</smiles>
MEMO<smiles>C=CCC1(C(C)(O)C(C)=C(F)F)CC1</smiles><smiles>C=CC1(CC(C)=O)CCCC1</smiles><smiles>C=CCC1(C(C)(O)C(F)=C(F)F)CCCC1</smiles><smiles>C#CCC1CCCCC1=O</smiles><smiles>C#CC[C@H]1CCCCC1(O)C(O)=C(F)F</smiles>

64

4.9:1 trans-3d/cis-3d

$42^{c, e}$

\section{trans-3d/cis-3d}

\section{3} trans $\mathbf{- 3} \mathbf{j} /$ cis-3j

\footnotetext{
${ }^{a}$ Isolated yields. All reactions were carried out with $12 \mathrm{mmol}$ of 1 unless otherwise stated. ${ }^{b}$ Diastereomeric ratio determined by ${ }^{19} \mathrm{~F}$ NMR spectroscopy; for mixtures the major isomer is shown. ${ }^{c}$ Isolated as a mixture of cis- and trans- diastereoisomers, major isomer shown. ${ }^{d}$ At 9.5 mmol scale. ${ }^{e}$ At 5 mmol scale. ${ }^{f}$ At 10 mmol scale. ${ }^{g}$ At 8 mmol scale.
} 
These allylic alcohols were indefinitely stable when stored at $0{ }^{\circ} \mathrm{C}$. With access to the requisite substrates secured, the allylic alcohol 3a was chosen as a model substrate to investigate the proposed novel cyclization. ${ }^{5}$ We began our studies using cyclization conditions similar to those reported by Toste for the carbocyclization of silyl enol ethers onto gold activated alkynes (Table 2). ${ }^{9}$

Table 2: Optimization of the one-pot cyclization reduction.

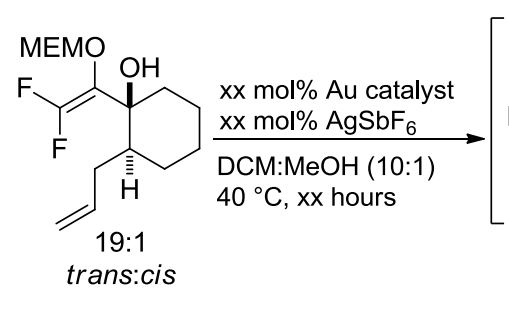

$3 a$

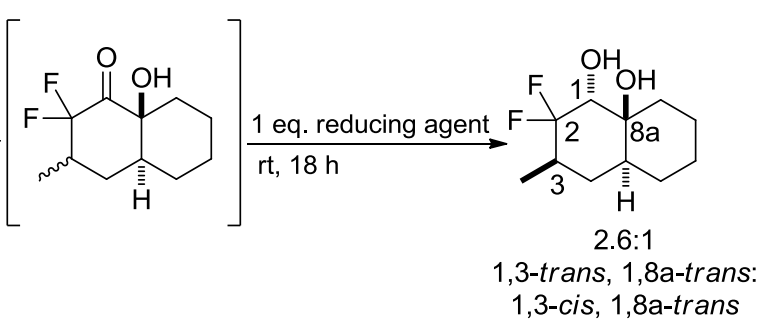

$4 a$

$\begin{array}{ccccccc} & \text { Au cat. } & \begin{array}{c}\text { Au cat. } \\ (\mathrm{mol} \%)\end{array} & \begin{array}{c}\mathrm{AgSbF}_{6} \\ \left(\mathrm{~mol}_{0}\right)\end{array} & \begin{array}{c}\text { time } \\ (\mathrm{h})\end{array} & \begin{array}{c}\text { reducing } \\ \text { agent }\end{array} & \begin{array}{c}\text { yield } \\ (\%)^{a}\end{array} \\ 1 & \mathrm{IPrAuCl} & 5 & 5 & 5 & \mathrm{NaBH}_{4} & 42 \\ 2 & \mathrm{IPrAuCl} & 5 & 5 & 5 & \mathrm{TBAB} & 70 \\ 3 & \mathrm{IPrAuCl} & 2.5 & 2.5 & 5 & \mathrm{TBAB} & 68 \\ 4 & \mathrm{IPrAuCl} & 1 & 1 & 22 & \mathrm{TBAB} & 51 \\ 5 & \mathrm{Ph}{ }_{3} \mathrm{PAuCl} & 5 & 5 & - & - & 0^{b} \\ 6 & \mathrm{IPrAuCl} & 5 & - & - & - & 0^{b} \\ 7 & \mathrm{~N} & - & 5 & - & - & 0^{b}\end{array}$

${ }^{a}$ Isolated Yield. Product was isolated as a mixture of 1,3-trans, 1,8a-trans and 1,3cis, 1,8a-trans diastereoisomers (2.6:1), Major isomer shown. ${ }^{b}$ No cyclized product could be detected by 19F NMR.

Using silver hexafluoroantimonate $(\mathrm{V})$ as a chloride abstractor (generating the catalytically active cationic gold species in situ) in conjunction with 5 mol\% 1,3-bis(2,6diisopropylphenyl-imidazol-2-ylidene)gold(I) chloride $(\mathrm{IPrAuCl})^{10}$ ensured a successful 
cyclization and the difluoroketone intermediate was reduced in situ using tetrabutylammonium borohydride (TBAB).

The improved solubility of TBAB over sodium borohydride in the reaction media may have accounted for the higher yield of $\mathbf{4 a}$ (Entry 2, 70\%, 2.6:1 dr).

Lower catalyst loadings resulted in diminished yields and more protracted purification procedures, whilst application of a phosphine based catalyst yielded no cyclized product. The necessary control reactions in the absence of both silver and gold catalyst were also performed. In both cases no cyclized product could be detected from the reaction indicating that both silver and gold components must be present in order for the cyclization to proceed smoothly. It is possible that the $\alpha$-hydroxy functionality may also provide some stereocontrol during the reduction process through dihydrogen bonding as no cis-diol was observed in the reaction mixture (Scheme 4). ${ }^{11}$
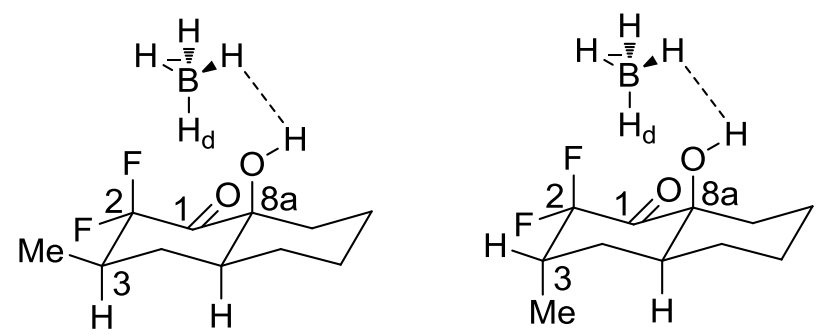

Scheme 4: Selective reduction affording 1,3-trans, 1,8a-trans 4a (left) and 1,3-cis, 1,8atrans 4a (right).

With optimized conditions in hand, we next investigated the scope of the one pot cyclization/reduction reaction (Table 3). 
Table 3: Scope of one-pot cyclization/reduction method.

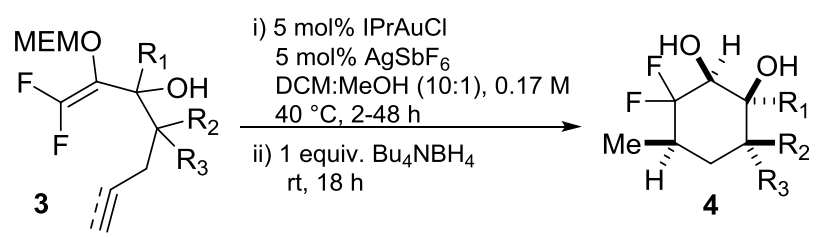

\begin{tabular}{|c|c|c|c|c|}
\hline & Allylic Alcohol & Product & Yield $(\%)^{a}$ & $\mathrm{dr}^{b}$ \\
\hline 1 & $\begin{array}{c}19: 1 \\
\text { trans-3a/cis-3a }\end{array}$ & & 70 & $\begin{array}{c}\mathbf{2 . 6}: \mathbf{1} \\
\text { 1, 3-trans, 1, 8a-trans } \\
1,3-\text {-cis, 1, 8a-trans }\end{array}$ \\
\hline 2 & $\begin{array}{c}32: 1 \\
\text { trans-3b/cis-3b }\end{array}$ & & 69 & $\begin{array}{c}\mathbf{4 . 9}: \mathbf{1} \\
\text { 1, 3-trans, 1, 9a-trans } \\
1,3-c i s, 1,9 \mathrm{a}-\text { trans }\end{array}$ \\
\hline 3 & $\begin{array}{c}3.5: 1 \\
\operatorname{trans} \mathbf{- 3} \mathbf{c} / \text { cis } \mathbf{- 3 c}\end{array}$ & & 48 & $\begin{array}{c}\text { 6.7:1.4:1 } \\
\text { 4a, 5-trans, 5, 7-trans } \\
\text { 4a, 5-trans, 5, 7-cis: } \\
\text { 4a, 5-cis, 5, 7-cis }\end{array}$ \\
\hline 4 & $\begin{array}{c}4.9: 1 \\
\text { trans }-\mathbf{3 d} / \text { cis-3d }\end{array}$ & & $50^{c}$ & $\begin{array}{c}\text { 3.4:0.9:1 } \\
\text { 4a, 5-trans, 5, 7-trans } \\
\text { 4a, 5-trans, 5, 7-cis: } \\
\text { 4a, 5-cis, 5, 7-cis }\end{array}$ \\
\hline 5 & $3 e$ & & $25^{d}$ & $\begin{array}{c}>\mathbf{2 0 : 1} \\
\text { 1, 2-trans, 2, 4-trans }\end{array}$ \\
\hline 6 & $3 e$ & & $16^{d}$ & $\begin{array}{c}>\mathbf{2 0 : 1} \\
1,2-\text { trans }, 2,4-c i s\end{array}$ \\
\hline 7 & 3f & & 11 & $\begin{array}{c}>\mathbf{2 0 : 1} \\
1,2-c i s, 2,4-\operatorname{trans}\end{array}$ \\
\hline 8 & $3 g$ & & 63 & $\begin{array}{c}>\mathbf{2 0 : 1} \\
1,2-c i s, 2,4-t r a n s\end{array}$ \\
\hline 9 & $3 h$ & & $29^{d}$ & $\begin{array}{c}\text { 1.3:1:0.1 } \\
\text { 4, 5-trans, 5, 7-cis: } \\
\text { 4, 5-trans, 5, 7-trans: } \\
\text { 4, 5-cis, 5, 7-cis }\end{array}$ \\
\hline 10 & $3 \mathbf{i}$ & 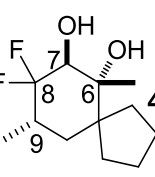 & 44 & $\begin{array}{c}\text { 3.8:1 } \\
\text { 6, 7-trans, 7, 9-trans: } \\
\text { 6, 7-cis, 7, 9-trans }\end{array}$ \\
\hline
\end{tabular}




11 trans-3j/cis-3j
${ }^{a}$ Isolated yield. ${ }^{b}$ Diastereomeric ratio determined by ${ }^{19} \mathrm{~F}$ NMR spectroscopy; for mixtures the major
isomer is shown. ${ }^{c} 10$ days reaction time. ${ }^{d}$ Diastereoisomers could be separated by normal phase
chromatography. ${ }^{e}$ From corresponding alkyne. 15 minutes reaction time and TBAB not added.

Cyclizations were typically performed on $1 \mathrm{mmol}$ scale. Annelations afforded a range of fused bicyclic difluorinated diols $\mathbf{4 a - 4 d}$ and the reaction also tolerated the formation of heterocycles 4c-4d. More challenging annulations could also be performed on both unsubstituted and substituted cyclization precursors. It was possible to isolate the single trans, trans diastereoisomers of $\mathbf{4 a}$ and $\mathbf{4 b}$ by recrystallization of the mixtures by vapor diffusion using chloroform/pentane. We were able to isolate diastereoisomerically pure trans, trans 4ea and trans, cis $\mathbf{4 e b}$ as well as a mixture of both the cis,cis and trans, trans diastereoisomers (1.2:1 respectively, $15 \%)$ representing an overall yield of $56 \%$. Annulations generally required a minimum level of substitution on the chain and a lower yield was obtained for the least substituted system $\mathbf{4 f}$ (11\%). Gem-dimethyl $\mathbf{4 g}$ was isolated in good yield and diastereoselectivity. In this case the selectivity of the reduction can be rationalized from consideration of the bulky borohydride reagent preferentially attacking the proequatorial face of the ketone, avoiding an unfavorable steric interaction with the axial C-3 methyl substituent of the ring. ${ }^{12}$ Consequently, the carbon center which is reduced now bears an $S$ configuration. Spirocycles $4 \mathbf{h}$ and $\mathbf{4 i}$ were both isolated as mixtures of diastereoisomers in moderate yield and for the spiro-cyclopropyl analogue, it was also possible to isolate small quantities of both trans, trans $4 \mathrm{~h}(5 \%)$ and trans, cis $\mathbf{4 h}(6 \%)$ as single diastereoisomers (representing an overall yield of $40 \%$ ). The stereochemical outcome of the reduction of spirocyclopentyl $\mathbf{4 i}$ is equivalent to gem-dimethyl $\mathbf{4 g}$ and the two diastereoisomers formed both have $S$ configuration at the reduced carbon center. Conversely, the three diastereoisomers 
isolated for $\mathbf{4 h}$ have the opposite $R$ configuration. This can be rationalized by consideration of the position of the 'axial' methylene group in $\mathbf{4 h}$. The strained cyclopropane ring requires bond angles to be smaller than those of typical $s p^{3}$ hybridized carbons; therefore, the methylene is not adopting a defined axial position, allowing the borohydride reagent to attack the pro-axial face of the ketone. The three-dimensional nature of such spirocyclic derivatives are of particular interest in medicinal chemistry and drug discovery. ${ }^{13}$ In reactions where multiple diastereoisomers were formed there structures were elucidated through a combination of X-ray crystallographic analysis, 2D NMR and ${ }^{19} \mathrm{~F}-{ }^{1} \mathrm{H}$ HOESY spectroscopic experiments. $^{14}$

Attempted cyclization of a terminal alkyne substrate did not yield the desired product and instead afforded non-fluorinated pyran 5. A working hypothesis as to how this product may be obtained is outlined in Scheme 5. We believe that initial attack of methanol onto the gold activated alkyne followed by a cyclization/elimination cascade is responsible for the formation of 5 .
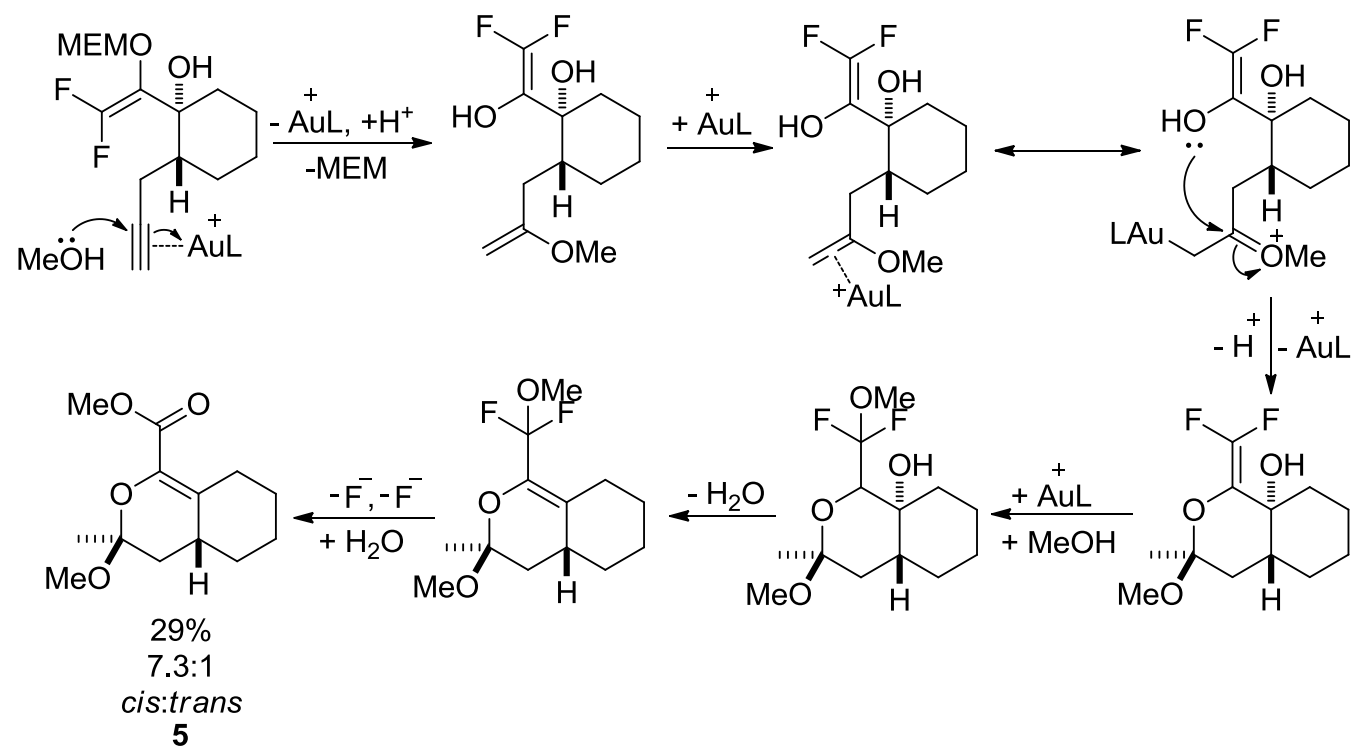<smiles>COC(F)(F)C1=C2CCCC[C@H]2C[C@](C)(OC)O1</smiles>

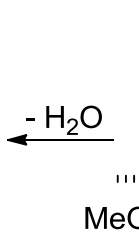<smiles>COC(F)(F)C1O[C@](C)(O)C[C@H]2CCCC[C@@]12O</smiles>

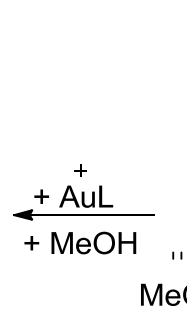<smiles>[3H][13C]([3H])=[14C]</smiles><smiles>O[C@]12CCCC[C@H]1C[C@@H](I)OC2=C(F)F</smiles>

5

Scheme 5. Proposed mechanism for formation of 5. 
Although unexpected this result was encouraging and we hypothesized that we could utilize the enhanced reactivity of the alkyne functionality to synthesize alternative difluorinated scaffolds.

Investigation into alkyne cyclization. Pyrans are becoming an increasingly important heterocyclic scaffold in medicinal chemistry and can be found in a variety of bioactive compounds. ${ }^{15}$ Only slight modification of the current synthetic route would enable access to structurally unique difluorinated pyran variants (Scheme 6)

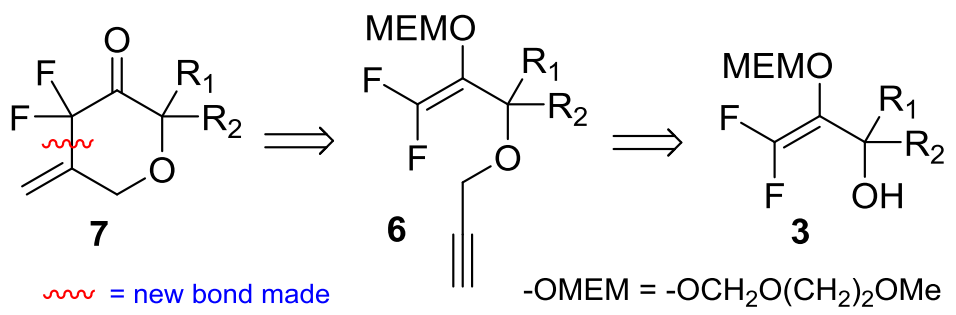

Scheme 6. Retrosynthetic route to difluorinated pyrans.

A small palette of propargyl ethers 6 were synthesized from difluoroallylic alcohols 3 using our published propargylation procedure $\left(\right.$ Table 4). ${ }^{(6 a)}$ 
Table 4 - Propargyl ethers 6 synthesized from allylic alcohols 3.
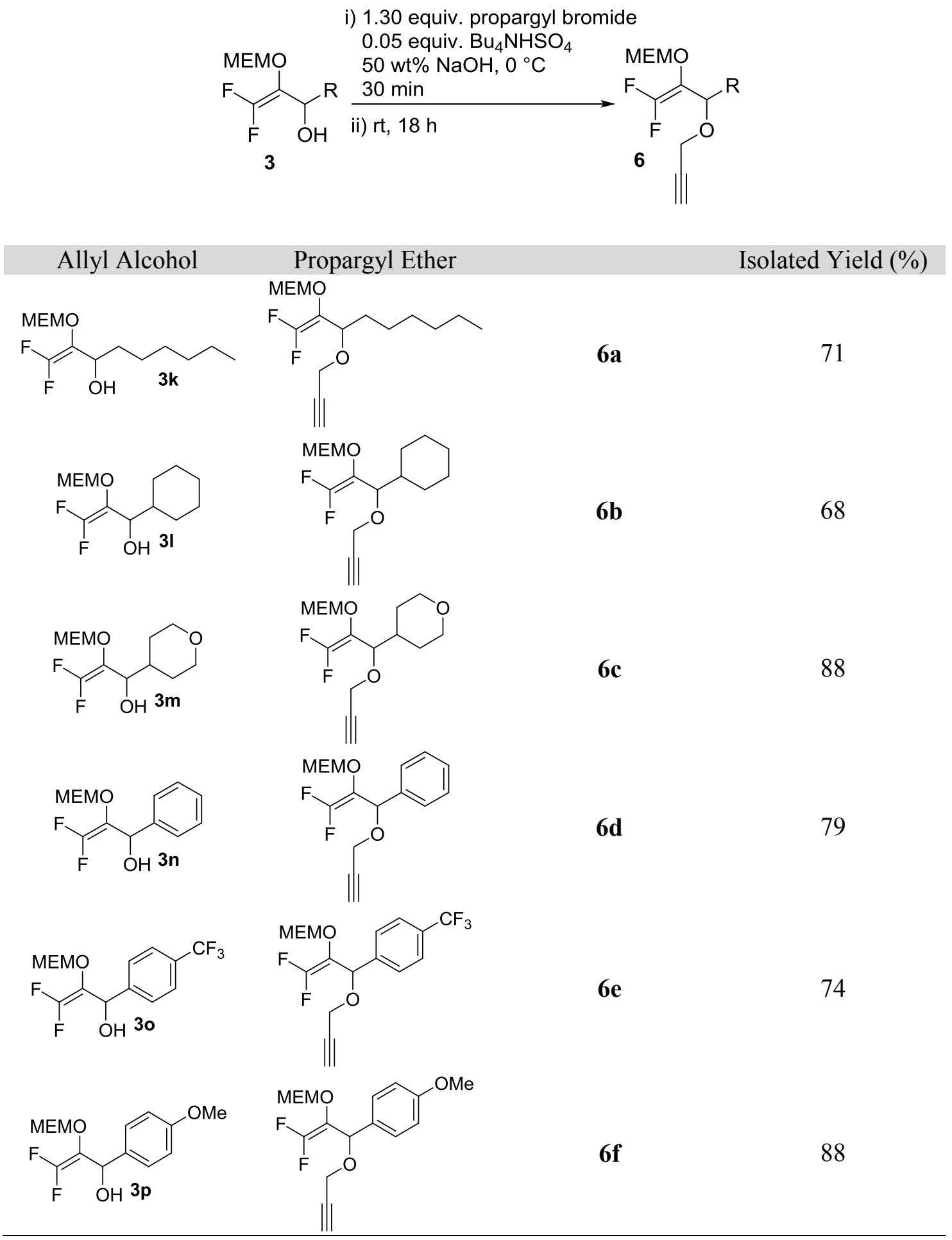
Simply treating difluoroallylic alcohols 3 with slight excess of propargyl bromide in a 50\% aqueous solution of sodium hydroxide and a phase transfer catalyst afforded the desired propargyl ethers in good yield. Alkyne 6a was chosen as a model substrate to investigate the proposed cyclization. Knowing that the alkyne moiety was susceptible to attack from methanol, the previously established cyclization conditions were employed in the absence of this solvent (Table 5).

Table 5: Optimisation of alkyne cyclization.

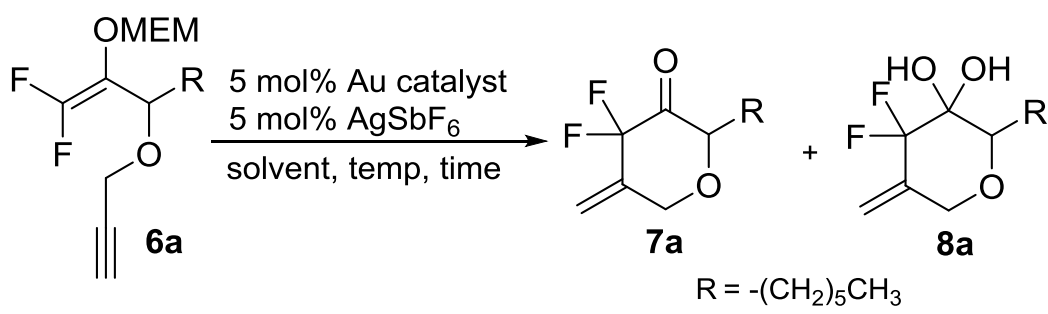

\begin{tabular}{cccccc} 
& Au catalyst & Temp $\left({ }^{\circ} \mathrm{C}\right)$ & Solvent & $\begin{array}{c}\text { Time } \\
(\mathrm{h})\end{array}$ & Isolated Yield $(\%)^{a}$ \\
1 & IPrAuCl & $\mathrm{rt}$ & DCM & 4 & 21 \\
2 & $\mathrm{IPrAuCl}$ & $\mathrm{rt}$ & Toluene & 5 & 61 \\
3 & $\mathrm{IPrAuCl}$ & $\mathrm{rt}$ & $\mathrm{THF}$ & 3 & 0 \\
4 & $\mathrm{IPrAuCl}$ & $\mathrm{rt}$ & 2-MeTHF & 21 & 65 \\
5 & $\mathrm{IPrAuCl}$ & $\mathrm{rt}$ & $\mathrm{CPME}$ & 5 & 61 \\
6 & $\mathrm{IPrAuCl}$ & 40 & 2-MeTHF & 2 & 49 \\
7 & $\mathrm{Ph}$ PAuCl & $\mathrm{rt}$ & 2-MeTHF & 24 & 54 \\
\hline${ }^{a}$ Yield calculated based on the molecular weight of the & hydrate component. ${ }^{b}$ Polymerisation \\
observed.
\end{tabular}

Using dichloromethane in a single solvent system it was possible to isolate the desired cyclized product, albeit in low yield. Given that $\alpha, \alpha$-difluoroketones are known to readily hydrate the products were often isolated as a mixture of the ketone $7 \mathbf{a}$ and corresponding hydrate 8a. ${ }^{17}$ Upon switching to toluene the reaction was complete after 5 hours, and a significant increase in yield was observed. Whilst toluene appeared suitable for this particular substrate we found it to be incompatible with others and instead formed complex mixtures. As the objective was to identify a generic solvent which was not just substrate specific we decided to continue with further solvent screening. Polymerization of the reaction mixture 
was observed when tetrahydrofuran was used. Tetrahydrofuran is known to undergo polymerization in the presence of catalytic quantities of metal halide salts, therefore the $\mathrm{AgSbF}_{6}$ used in the reaction is likely to be the trigger for this process. ${ }^{17}$ This unwanted polymerization could be avoided by switching to 2-methyltetrahydrofuran (2-MeTHF). Pleasingly, this solvent was not substrate specific and was compatible with other cyclizations. The ability to conduct the reaction in this inexpensive, sustainable solvent at room temperature is an attractive feature of the methodology. ${ }^{18}$ Using cyclopentyl methyl ether (CPME) as an alternative ethereal solvent gave similar results to those obtained in toluene and also proved ineffective with other substrates screened. Following this screen we decided to use 2-MeTHF as the solvent of choice for moving forward with the investigation. Increasing the reaction temperature significantly decreased the reaction time; however, this was accompanied by a lower product yield. $\mathrm{Ph}_{3} \mathrm{PAuCl}$ could also be used as a catalyst for the reaction; however, the best results were achieved by using IPrAuCl.

The reaction tolerated a variety of substituents. Carbocyclic analogue $\mathbf{8 b}$ was isolated in comparable yield to the model substrate and, in this case, the ketone existed as the major component in the mixture. In contrast heterocycle $\mathbf{8 c}$ was isolated exclusively as the hydrate. This material was recrystallized by vapor diffusion using tetrahydrofuran/pentane and its structure confirmed by single crystal X-ray diffraction. It was also pleasing to note that the reaction tolerated aromatic substituents. Phenyl analogue 8d and electron withdrawing (trifluoromethyl) phenyl 8e were isolated in good yield. This result was encouraging as we had previously been unable to access such cyclic difluorinated systems bearing aromatic substituents. ${ }^{5}$ Electron withdrawing aromatic substituents were tolerated; however, we were unable to isolate electron rich aromatic $\mathbf{8 f}$ and the reaction yielded only a complex mixture (Table 6). 
Table 6 - Scope of Alkyne Cyclization.

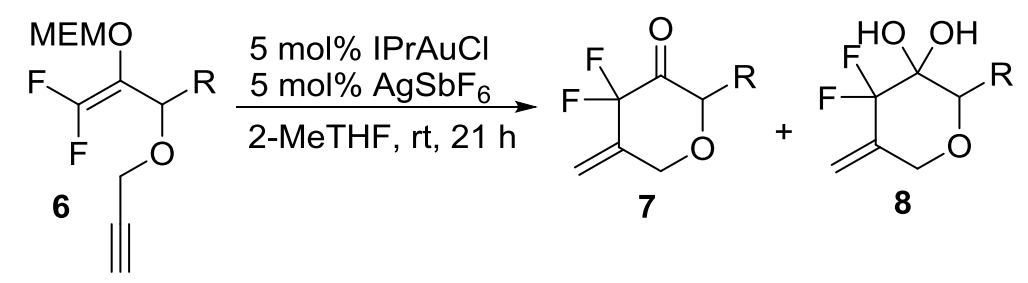

Isolated $\mathrm{Yield}^{a}$ Ketone:Hydrate $^{b}$

\footnotetext{
${ }^{a}$ Isolated yield. Yield is based upon major component. ${ }^{b}$ Ratio determined by ${ }^{19} \mathrm{~F}$ NMR spectroscopy; in each example the hydrate component is shown. ${ }^{c}$ Yield calculated based on the molecular weight of the hydrate component. ${ }^{d}$ Yield calculated based on the molecular weight of the ketone component.
}

\section{Conclusion}

The methodology described allows for the concise synthesis of either difluorinated diol or difluorinated pyran scaffolds (3 steps and 4 steps respectively from trifluoroethanol, an 
inexpensive commercial feedstock). Cyclizations are simple to perform and complex diol fragments can be synthesized via a one pot cyclization/reduction method under mild conditions in reactions vessels open to air. Alkyne cyclizations can be performed under similarly mild conditions at room temperature in 2-Methyltetrahydrofuran to afford products rich in functionality and $s p^{3}$ character and inaccessible by concise methods.

\section{Experimental Section}

General Methods. NMR spectra were recorded on Bruker DPX-400, AV-500 and AvanceII +600 spectrometers. ${ }^{1} \mathrm{H},{ }^{19} \mathrm{~F}$ and ${ }^{13} \mathrm{C}$ NMR spectra were recorded using the deuterated solvent as the lock and the residual solvent as the internal reference. The multiplicities of the spectroscopic data are presented in the following manner: $\mathrm{s}=$ singlet, $\mathrm{d}=$ doublet, $\mathrm{dd}=$ double doublet, $\mathrm{dt}=$ doublet of triplets, $\mathrm{dq}=$ doublet of quartets, dquint $=$ doublet of quintets, $\mathrm{ds}=$ doublet of sextets, $\mathrm{qd}=$ quartet of doublets, $\mathrm{qt}=$ quartet of triplets, $\mathrm{ddt}=$ doublet of double triplets, $\mathrm{ddd}=$ doublet of doublet of doublets, dddd $=$ doublet of double doublet of doublets, dddt $=$ doublet of double doublet of triplets, $\mathrm{tdd}=$ triplet of doublet of doublets, $\mathrm{td}=$ triplet of doublets, $\mathrm{t}=$ triplet, $\mathrm{q}=$ quartet, $\mathrm{m}=$ multiplet and $\mathrm{br}$. $=$ broad. Unless stated otherwise, all couplings refer to ${ }^{3} J$ homocouplings. IR spectra were recorded on an ATR IR spectrometer. GC/MS spectra were obtained on an instrument fitted with a DB5-type column $(30 \mathrm{~m} \times 0.25 \mu \mathrm{m})$ running a $40-320{ }^{\circ} \mathrm{C}$ temperature program, ramp rate $20{ }^{\circ} \mathrm{C} \mathrm{min}{ }^{-1}$ with helium carrier gas flow at $1 \mathrm{~cm}^{3} \mathrm{~min}^{-1}$. Chemical ionisation (CI) (methane/ammonia) and Electron Ionisation (EI) mass spectra were recorded on either an Agilent Technologies 5975C mass spectrometer or a FINNIGAN MAT 95 high resolution double focussing (BE) mass spectrometer (EPSRC National Mass Spectrometry Service Centre, Swansea). HRMS measurements were obtained from a Waters GCT Premier MS (CI), Finnigan Mat 95 XP (EIMS and/or APCI-MS), Thermo Scientific LTQ Orbitrap XL via Advion TriVersa NanoMate infusion (ESI) or Waters Xevo G2-S Atmospheric Solids Analysis Probe (APCI, Positive 
mode, Xevo) spectrometers (EPSRC National Mass Spectrometry Service Centre, Swansea). Thin layer chromatography was performed on pre-coated aluminium-backed silica gel plates (E. Merck AG, Darmstadt, Germany. Silica gel 60 F254, thickness $0.2 \mathrm{~mm}$ ). Visualisation was achieved using potassium permanganate dip or UV detection at $254 \mathrm{~nm}$. Column chromatography was performed on silica gel (Zeochem, Zeoprep 60 HYD, 40-63 $\mu \mathrm{m}$ ) using a Büchi Sepacore system. Hexane was distilled before chromatography. [(IPr)AuCl] (IPr = 1,3-bis(2,6-diisopropylphenyl)imidazole-2-ylidene, 95\%) and $\mathrm{AgSbF}_{6}(98 \%)$ were purchased from Strem Chemicals and used as received. THF was dried using a PureSolv system from Innovative Technology, Inc.. Diisopropylamine was distilled from $4 \AA$ molecular sieves (30 ${ }^{\circ} \mathrm{C} / 140$ mbar) and stored under nitrogen over $4 \AA$ molecular sieves. All other chemicals were purchased from Sigma Aldrich, Alfa Aesar, or Fluorochem. All compounds were named according to the ChemDraw Professional 15.0 package and checked against Scifinder ${ }^{\circledR}$ chemical database. Single-crystal data were measured at with Oxford Diffraction CCD Diffractometers and with sealed-tube generated, graphite monochromated radiation. The exception was $\mathbf{7 c}$ for which data was measured by the UK National Crysallography Service using a Rigaku FRE+ rotating anode. ${ }^{19}$ The structures were solved by direct methods (SIR92, SHELXS) and refined to convergence on $F^{2}$ and against all independent reflections by fullmatrix least-squares using SHELXL programs..$^{20,21}$ All non-hydrogen atoms were refined anisotropically and hydrogen atoms were geometrically placed and allowed to ride on their parent atoms. For compound trans, trans-4a, crystals grew as stacked plates. Reprocessing the raw data for this compound as a twinned sample gave a hklf 5 format reflection file. Refinement with this gave significantly better R factors and residual electron density features with BASF $=0.265(23)$. Table S1 contains selected crystallographic and refinement data. CCDC-1582563 to CCDC-1582570 contain the supplementary crystallographic data for this paper. These data can be obtained free of charge from the Cambridge Crystallographic Data 
Centre via www.ccdc.cam.ac.uk/data_request/cif. LDA and $n$-Butyllithium (2.5 M solution in hexanes) was titrated according to the method of Duhamel and Plaquevent. ${ }^{22}$ The data for products $\mathbf{3 f}, \mathbf{3 g}, \mathbf{3 n}$, and $\mathbf{3 p}$ have previously been reported. . $^{6,8,23}$

General Procedure A: Allylic Alcohol Preparation. Trans-2-allyl-1-(2,2-difluoro-1 ((2methoxyethoxy)methoxy)ethenyl) cyclohexan-1-ol (trans-3a) and cis-2-allyl-1-(2,2-difluoro1' ((2-methoxyethoxy)methoxy)ethenyl) cyclohexan-1-ol (cis-3a). Prepared according to the method of Percy and co-workers. ${ }^{8}$ n-Butyllithium $(12.9 \mathrm{~mL}$ of a $1.94 \mathrm{M}$ solution in hexanes, $25 \mathrm{mmol})$ was added dropwise to a solution of diisopropylamine $(3.70 \mathrm{~mL}, 26 \mathrm{mmol})$ in THF $(12 \mathrm{~mL})$ at $-78{ }^{\circ} \mathrm{C}$. Following addition the reaction vessel was transferred to an ice bath and allowed to warm to $0{ }^{\circ} \mathrm{C}$ and stirred at this temperature for 45 minutes. The flask was then recooled to $-78^{\circ} \mathrm{C}$ and acetal $\mathbf{1}^{8}(1.90 \mathrm{~mL}, 12 \mathrm{mmol})$ was added dropwise over 15 minutes. The dark orange suspension was stirred at $-78{ }^{\circ} \mathrm{C}$ for 30 minutes, then allylcyclohexanone $(1.97$ $\mathrm{mL}, 13.2 \mathrm{mmol}$ ) was added. The reaction mixture was allowed to warm to rt overnight; the reaction mixture turned homogeneous and darkened over this time. The mixture was quenched with saturated aqueous ammonium chloride $(30 \mathrm{~mL})$ and extracted with ethyl acetate $(4 \times 60 \mathrm{~mL})$. The combined organic extracts were dried $\left(\mathrm{MgSO}_{4}\right)$, filtered and concentrated to afford crude allylic alcohols trans-3a and cis-3a (3.23 g) as a dark brown oil. The material was taken up in dichloromethane and transferred by pipette to a glass sinter funnel (diameter $7.5 \mathrm{~cm}$ ) containing a pad of silica $(54 \mathrm{~g})$. The product was then eluted from the plug with $70 \%$ diethyl ether/hexane $(600 \mathrm{~mL})$. The solvent was evaporated under reduced pressure to afford the product as a pale orange oil $(2.93 \mathrm{~g})$. The crude allylic alcohol was purified by Kugelrohr distillation to afford an inseparable mixture of trans-3a and cis-3a as a pale yellow oil (2.53 g, $69 \%$, 95:5). b.p. $=97^{\circ} \mathrm{C} / 0.04 \mathrm{mmHg} ; \mathrm{R}_{\mathrm{f}}=0.27$ (40\% diethyl ether in hexane); ${ }^{1} \mathrm{H}$ NMR $\left(400 \mathrm{MHz}, \mathrm{CDCl}_{3}\right): \delta=5.77$ (dddd, $\left.\mathrm{J}=17.0,10.1,8.5,5.8 \mathrm{~Hz}, 1 \mathrm{H}\right)$, 5.06-4.95 (m, 2H), 4.93 (app. s, 2H), 3.94-3.81 (m, 2H), 3.62-3.54 (m, 2H), 3.40 (s, 3H), 2.62 
(br. s, $1 \mathrm{H}), 2.29-2.16(\mathrm{~m}, 1 \mathrm{H}), 1.98-1.88(\mathrm{~m}, 1 \mathrm{H}), 1.86-1.08$ (envelope, $9 \mathrm{H}) \mathrm{ppm} ;{ }^{13} \mathrm{C} \mathrm{NMR}$ $\left(100 \mathrm{MHz}, \mathrm{CDCl}_{3}\right): \delta=154.4\left(\mathrm{t},{ }^{1} \mathrm{~J}_{\mathrm{C}-\mathrm{F}}=287.1 \mathrm{~Hz}\right), 137.6,121.5\left(\mathrm{dd},{ }^{2} \mathrm{~J}_{\mathrm{C}-\mathrm{F}}=33.1,10.3 \mathrm{~Hz}\right)$, $115.8,98.7\left(\mathrm{t},{ }^{4} \mathrm{~J}_{\mathrm{C}-\mathrm{F}}=4.2 \mathrm{~Hz}\right), 73.7\left(\mathrm{~d},{ }^{3} \mathrm{~J}_{\mathrm{C}-\mathrm{F}}=5.6 \mathrm{~Hz}\right), 71.6,68.9,58.9,41.9\left(\mathrm{~d},{ }^{4} \mathrm{~J}_{\mathrm{C}-\mathrm{F}}=4.8\right.$ $\mathrm{Hz}), 37.1\left(\mathrm{t},{ }^{4} \mathrm{~J}_{\mathrm{C}-\mathrm{F}}=2.9 \mathrm{~Hz}\right), 35.2,26.4,25.3,21.2 \mathrm{ppm}$; Major trans-diastereoisomer $\mathbf{3 a}$ (assigned on the basis of $\delta$ and intensity) ${ }^{19} \mathrm{~F}$ NMR (376 MHz, $\left.\mathrm{CDCl}_{3}\right): \delta=-98.8\left(\mathrm{~d},{ }^{2} \mathrm{~J}=73.4\right.$ $\mathrm{Hz}, 1 \mathrm{~F}),-103.6\left(\mathrm{~d},{ }^{2} \mathrm{~J}=73.4 \mathrm{~Hz}, 1 \mathrm{~F}\right) \mathrm{ppm}$;* Minor cis-diastereoisomer 3a (assigned on the basis of $\delta$ and intensity) ${ }^{19} \mathrm{~F}$ NMR $\left(376 \mathrm{MHz} \mathrm{CDCl}_{3}\right): \delta=-97.5\left(\mathrm{~d},{ }^{2} \mathrm{~J}=68.6 \mathrm{~Hz}, 1 \mathrm{~F}\right), 104.4$ $\left(\mathrm{d},{ }^{2} \mathrm{~J}=68.6 \mathrm{~Hz}, 1 \mathrm{~F}\right) \mathrm{ppm} ; \bar{v} /($ neat $)=3450,2934,1738,1450,1104,1059 \mathrm{~cm}^{-1}$; HRMS (NSI): calcd for $\mathrm{C}_{15} \mathrm{H}_{28} \mathrm{~F}_{2} \mathrm{O}_{4} \mathrm{~N}, 324.1981\left[\mathrm{M}+\mathrm{NH}_{4}\right]^{+}$, found: 324.1983; MS (CI): m/z (\%): 201 (14) $\left[\mathrm{M}-\mathrm{C}_{4} \mathrm{H}_{9} \mathrm{O}_{3}\right]^{+}, 89(100)\left[\mathrm{C}_{4} \mathrm{H}_{9} \mathrm{O}_{2}\right]^{+}, 59(85)\left[\mathrm{C}_{3} \mathrm{H}_{7} \mathrm{O}\right]^{+} ; \mathrm{t}_{\mathrm{R}}(\mathrm{GC})=13.17$ minutes**. *this is by comparison with compounds from the Saegusa-Ito series..$^{5 *}$ the cis- and trans-stereoisomers appeared as one peak by GC.

trans-2-allyl-1-(2,2-difluoro-1-((2-methoxyethoxy)methoxy)vinyl)cycloheptan-1-ol (trans-3b) and cis-2-allyl-1-(2,2-difluoro-1-((2-methoxyethoxy)methoxy)vinyl)cycloheptan1-ol (cis-3b). Prepared as for trans-3a and cis-3a from acetal $(1.50 \mathrm{~mL}, 9 \mathrm{mmol}), \mathrm{n}-$ butyllithium (12.8 $\mathrm{mL}$ of a $1.48 \mathrm{M}$ solution in hexanes, $19 \mathrm{mmol})$, diisopropylamine $(2.85$ $\mathrm{mL}, 20 \mathrm{mmol})$ and allylcycloheptanone $\mathrm{s}^{5}(1.45 \mathrm{~g}, 9.5 \mathrm{mmol})$ in THF $(12 \mathrm{~mL})$. The crude allylic alcohol (3.13 g) was purified by Kugelrohr distillation to afford an inseparable mixture of trans-3b and cis-3b as a pale yellow oil $(0.99 \mathrm{~g}, 32 \%, 97: 3)$. b.p. $=93{ }^{\circ} \mathrm{C} / 0.04 \mathrm{mmHg} ; \mathrm{R}_{\mathrm{f}}$ $=0.27\left(40 \%\right.$ diethyl ether in hexane); ${ }^{1} \mathrm{H} \mathrm{NMR}\left(400 \mathrm{MHz}, \mathrm{CDCl}_{3}\right): \delta=5.79(\mathrm{dddd}, \mathrm{J}=16.9$, 10.2, 8.6, 5.2 Hz, 1H), 5.08-4.98 (m, 2H), 4.96, $4.92\left(\mathrm{ABq}, \mathrm{J}_{\mathrm{AB}}=6.1 \mathrm{~Hz}, 2 \mathrm{H}\right), 3.94-3.84(\mathrm{~m}$, 2H), 3.62-3.52 (m, 2H), 3.40 (s, 3H), 2.79 (br. s, 1H), 2.27-2.15 (m, 1H), 2.09-1.99 (m, 1H), 1.99-1.89 (m, 1H), 1.87-1.20 (envelope, $10 \mathrm{H}) \mathrm{ppm} ;{ }^{13} \mathrm{C}$ NMR (100 MHz, $\left.\mathrm{CDCl}_{3}\right): \delta=154.1$ $\left(\mathrm{t},{ }^{1} \mathrm{~J}_{\mathrm{C}-\mathrm{F}}=287.5 \mathrm{~Hz}\right), 137.6,121.9\left(\mathrm{dd},{ }^{2} \mathrm{~J}_{\mathrm{C}-\mathrm{F}}=32.2,10.2 \mathrm{~Hz}\right), 115.5,98.3\left(\mathrm{t},{ }^{4} \mathrm{~J}_{\mathrm{C}-\mathrm{F}}=4.2 \mathrm{~Hz}\right)$, $76.3\left(\mathrm{~d},{ }^{3} \mathrm{~J}_{\mathrm{C}-\mathrm{F}}=6.0 \mathrm{~Hz}\right), 71.1,68.5,58.5,45.1\left(\mathrm{~d},{ }^{4} \mathrm{~J}_{\mathrm{C}-\mathrm{F}}=5.2 \mathrm{~Hz}\right), 39.4,35.8,27.7,26.8,24.9$, 
20.0 ppm; Major trans-diastereoisomer 3b (assigned on the basis of $\delta$ and intensity) ${ }^{19} \mathrm{~F}$ NMR $\left(376 \mathrm{MHz}, \mathrm{CDCl}_{3}\right): \delta=-98.6\left(\mathrm{~d},{ }^{2} \mathrm{~J}=74.1 \mathrm{~Hz}, 1 \mathrm{~F}\right),-103.9\left(\mathrm{~d},{ }^{2} \mathrm{~J}=74.1 \mathrm{~Hz}, 1 \mathrm{~F}\right) \mathrm{ppm} ;{ }^{*}$ Minor cis-diastereoisomer 3b (assigned on the basis of $\delta$ and intensity) ${ }^{19} \mathrm{~F} \mathrm{NMR} \mathrm{(376} \mathrm{MHz,}$ $\left.\mathrm{CDCl}_{3}\right): \delta=-97.7\left(\mathrm{~d},{ }^{2} \mathrm{~J}=72.6 \mathrm{~Hz}, 1 \mathrm{~F}\right), 103.0\left(\mathrm{~d},{ }^{2} \mathrm{~J}=72.6 \mathrm{~Hz}, 1 \mathrm{~F}\right) \mathrm{ppm} ; \overline{\mathrm{v}} /($ neat $)=3464$, 2921, 1733, 1262, 1050, $952 \mathrm{~cm}^{-1}$; HRMS (APCI): calcd for $\mathrm{C}_{16} \mathrm{H}_{30} \mathrm{~F}_{2} \mathrm{O}_{4} \mathrm{~N}_{1}, 338.2137$ $\left[\mathrm{M}+\mathrm{NH}_{4}\right]^{+}$, found: $338.2140 ; \mathrm{MS}(\mathrm{CI}): \mathrm{m} / \mathrm{z}(\%): 338(100)\left[\mathrm{M}+\mathrm{NH}_{4}\right]^{+} ; \mathrm{t}_{\mathrm{R}}(\mathrm{GC})=14.17$ minutes. ${ }^{* *}$ this is by comparison with compounds from the Saegusa-Ito series. ${ }^{5 * *}$ the cisand trans-stereoisomers appeared as one peak by GC.

trans-3-allyl-4-(2,2-difluoro-1-((2-methoxyethoxy)methoxy)vinyl)tetrahydro-2H-

pyran-4-ol (trans-3c) and cis-3-allyl-4-(2,2-difluoro-1-((2methoxyethoxy)methoxy)vinyl)tetrahydro-2H-pyran-4-ol (cis-3c). Prepared as for trans-3a and cis-3a from acetal $(1.90 \mathrm{~mL}, 12 \mathrm{mmol}), n$-butyllithium $(12.9 \mathrm{~mL}$ of a $1.94 \mathrm{M}$ solution in hexanes, $25 \mathrm{mmol})$, diisopropylamine $(3.70 \mathrm{~mL}, 26 \mathrm{mmol})$ and 2-allyltetrahydropyranone ${ }^{5}$ (1.68 g, $12 \mathrm{mmol})$ in THF (12 mL). The crude allylic alcohol (3.13 g) was purified by Kugelrohr distillation to afford an inseparable mixture of trans-3c and cis-3c as a pale yellow oil (2.06 g, $56 \%$, 78:22). b.p. $=93{ }^{\circ} \mathrm{C} / 0.04 \mathrm{mmHg} ; \mathrm{R}_{f}=0.25$ (30\% ethyl acetate in hexane); Major trans-diastereoisomer 3c (assigned on the basis of $\delta$ and intensity) ${ }^{1} \mathrm{H}$ NMR (400 MHz, $\left.\mathrm{CDCl}_{3}\right): \delta=5.90-5.65(\mathrm{~m}, 1 \mathrm{H}), 5.21-4.81$ (m, including 4.96 (app. s, 2H) 2H), 4.03-3.67 (envelope, 5H), $3.60(\mathrm{t}, J=4.7 \mathrm{~Hz}, 2 \mathrm{H}), 3.57-3.49(\mathrm{~m}, 1 \mathrm{H}), 3.41(\mathrm{~s}, 3 \mathrm{H}), 3.16$ (br. $\mathrm{s}, 1 \mathrm{H})$, 2.29-1.39 (envelope, 5H) ppm; ${ }^{13} \mathrm{C} \mathrm{NMR}\left(100 \mathrm{MHz}, \mathrm{CDCl}_{3}\right): \delta=153.9\left(\mathrm{t},{ }^{1} J_{\mathrm{C}-\mathrm{F}}=288.1 \mathrm{~Hz}\right)$, $135.8,120.5\left(\mathrm{dd},{ }^{2} J_{\mathrm{C}-\mathrm{F}}=32.8,10.4 \mathrm{~Hz}\right), 115.9,98.6\left(\mathrm{t},{ }^{4} J_{\mathrm{C}-\mathrm{F}}=4.4 \mathrm{~Hz}\right), 71.0,70.5\left(\mathrm{~d},{ }^{3} J_{\mathrm{C}-\mathrm{F}}=\right.$ $5.7 \mathrm{~Hz}), 68.6,66.7,62.9,58.4,40.5\left(\mathrm{~d},{ }^{4} J_{\mathrm{C}-\mathrm{F}}=5.1 \mathrm{~Hz}\right), 36.6,30.4 \mathrm{ppm} ;{ }^{19} \mathrm{~F}$ NMR $(376 \mathrm{MHz}$, $\left.\mathrm{CDCl}_{3}\right): \delta=-98.0\left(\mathrm{~d},{ }^{2} J=71.8 \mathrm{~Hz}, 1 \mathrm{~F}\right),-103.3\left(\mathrm{~d},{ }^{2} J=71.8 \mathrm{~Hz}, 1 \mathrm{~F}\right)$ ppm; ${ }^{*}$ Minor cisdiastereoisomer $3 \mathbf{c}$ (assigned on the basis of $\delta$ and intensity) ${ }^{1} \mathrm{H}$ NMR $\left(400 \mathrm{MHz}, \mathrm{CDCl}_{3}\right): \delta=$ $3.41\left(\mathrm{~s}, \mathrm{OCH}_{3}, 3 \mathrm{H}\right) \mathrm{ppm} ;{ }^{13} \mathrm{C} \mathrm{NMR}\left(100 \mathrm{MHz}, \mathrm{CDCl}_{3}\right): \delta=155.0\left(\mathrm{t},{ }^{1} J_{\mathrm{C}-\mathrm{F}}=290.7 \mathrm{~Hz}\right), 136.4$, 
$121.4\left(\mathrm{dd},{ }^{2} J_{\mathrm{C}-\mathrm{F}}=31.2,12.0 \mathrm{~Hz}\right), 116.0,70.9,68.2,63.6,62.5\left(\mathrm{~d},{ }^{3} J_{\mathrm{C}-\mathrm{F}}=4.8 \mathrm{~Hz}\right), 41.7,40.0$, 31.7 ppm; ${ }^{19} \mathrm{~F}$ NMR $\left(376 \mathrm{MHz}, \mathrm{CDCl}_{3}\right): \delta=-96.7\left(\mathrm{~d},{ }^{2} J=66.4 \mathrm{~Hz}, 1 \mathrm{~F}\right), 104.5\left(\mathrm{dt},{ }^{2} J=68.6\right.$, $\left.{ }^{5} J_{\mathrm{F}-\mathrm{H}}=4.9 \mathrm{~Hz}, 1 \mathrm{~F}\right) \mathrm{ppm} ; \bar{v} /($ neat $)=3443,2928,1736,1100,1056 \mathrm{~cm}^{-1}$; HRMS (APCI): calcd for $\mathrm{C}_{14} \mathrm{H}_{26} \mathrm{~F}_{2} \mathrm{O}_{5} \mathrm{~N}, 326.1774\left[\mathrm{M}+\mathrm{NH}_{4}\right]^{+}$, found: 326.1772 ; MS (CI): $m / z(\%): 326$ (100) $\left[\mathrm{M}+\mathrm{NH}_{4}\right]^{+} ; \mathrm{t}_{\mathrm{R}}(\mathrm{GC})=13.73$ minutes (major trans-diastereoisomer), 13.66 minutes (minor cisdiastereoisomer). ${ }^{*}$ this is by comparison with compounds from the Saegusa-Ito series. ${ }^{5}$

trans-tert-butyl -3-allyl-4-(2,2-difluoro-1-((2-methoxyethoxy)methoxy)vinyl)-4hydroxypiperidine-1-carboxylate (trans-3d) and cis-tert-butyl-3-allyl-4-(2,2-difluoro-1-((2methoxyethoxy)methoxy)vinyl)-4-hydroxypiperidine-1-carboxylate (cis-3d). Prepared as for trans-3a and cis-3a from acetal $(0.79 \mathrm{~mL}, 5 \mathrm{mmol})$, commercial LDA $(5.9 \mathrm{~mL}$ of a $1.70 \mathrm{M}$ solution in THF/heptane/ethylbenzene, $24 \mathrm{mmol}$ ) and tert-butyl 3-allyl-4-oxopiperidine-1carboxylate $^{5}(1.19 \mathrm{~g}, 5 \mathrm{mmol})$ in THF $(5 \mathrm{~mL})$. The crude allylic alcohol was purified by flash column chromatography (90 g cartridge, $45 \%$ ethyl acetate in hexane) to afford an inseparable mixture of trans-3d and cis-3d as a pale orange oil $(0.86 \mathrm{~g}, 42 \%, 83: 17)$. $\mathrm{R}_{\mathrm{f}}=$ 0.59 (50 \% ethyl acetate in hexane); ${ }^{1} \mathrm{H}$ NMR (400 MHz, $\left.\mathrm{CDCl}_{3}\right): \delta=5.79$ (dddd, $J=17.3$, 10.1, 8.5, 5.7 Hz, 1H), 5.14-4.99 (m, 2H), $4.94(\mathrm{~s}, 2 \mathrm{H}), 4.17-3.71(\mathrm{~m}, 4 \mathrm{H}), 3.70-3.51(\mathrm{~m}, 2 \mathrm{H})$, $3.41(\mathrm{~s}, 3 \mathrm{H}), 3.31-3.04(\mathrm{~m}, 2 \mathrm{H}), 2.87$ (br. s, $1 \mathrm{H}), 2.34-2.18(\mathrm{~m}, 1 \mathrm{H}), 2.06-1.73(\mathrm{~m}, 4 \mathrm{H}), 1.47$ (s, 9H) ppm (the ${ }^{1} \mathrm{H}$ NMR gave a clear well resolved spectrum at $\mathrm{RT}$ and did not require heating). Major trans-diastereoisomer 3d (assigned on the basis of $\delta$ and intensity) ${ }^{13} \mathrm{C}$ NMR $\left(150 \mathrm{MHz}\right.$, toluene- $\left.d_{8}, 373 \mathrm{~K}\right): \delta=154.7\left(\mathrm{t},{ }^{1} J_{\mathrm{C}-\mathrm{F}}=288.1 \mathrm{~Hz}\right), 154.2,137.0,121.2\left(\mathrm{dd},{ }^{2} J_{\mathrm{C}-\mathrm{F}}\right.$ = 32.4. $10.7 \mathrm{~Hz}), 115.5,98.8\left(\mathrm{dd},{ }^{4} J_{\mathrm{C}-\mathrm{F}}=5.8,3.4 \mathrm{~Hz}\right), 78.4,72.1\left(\mathrm{~d},{ }^{3} J_{\mathrm{C}-\mathrm{F}}=5.2 \mathrm{~Hz}\right), 71.5$, 69.0, 58.0, 44.0, $41.3\left(\mathrm{~d},{ }^{4} J_{\mathrm{C}-\mathrm{F}}=4.2 \mathrm{~Hz}\right), 39.6,36.5\left(\mathrm{t},{ }^{4} J_{\mathrm{C}-\mathrm{F}}=3.2 \mathrm{~Hz}\right), 31.9,28.1 \mathrm{ppm} ;{ }^{19} \mathrm{~F}$ $\left(376 \mathrm{MHz}\right.$, toluene- $\left.d_{8}, 373 \mathrm{~K}\right): \delta=-98.6\left(\mathrm{~d},{ }^{2} J=74.3 \mathrm{~Hz}, 1 \mathrm{~F}\right),-104.6\left(\mathrm{~d},{ }^{2} J=74.3 \mathrm{~Hz}, 1 \mathrm{~F}\right)$ ppm;* Minor cis-diastereoisomer 3d (assigned on the basis of $\delta$ and intensity) ${ }^{13} \mathrm{C}$ NMR (150 MHz, toluene- $\left.d_{8}, 373 \mathrm{~K}\right): \delta=155.7\left(\mathrm{t},{ }^{1} J_{\mathrm{C}-\mathrm{F}}=289.3 \mathrm{~Hz}\right), 154.6,136.4,115.8,78.3,72.4(\mathrm{dd}$, 
$\left.{ }^{3} J_{\mathrm{C}-\mathrm{F}}=4.6,2.3 \mathrm{~Hz}\right), 71.4,68.8,50.1,42.9,40.9,39.7,32.1,28.1 \mathrm{ppm} ;{ }^{19} \mathrm{~F}$ (376 MHz, toluene$d 8,373 \mathrm{~K}): \delta=-97.5\left(\mathrm{~d},{ }^{2} J=69.1 \mathrm{~Hz}, 1 \mathrm{~F}\right),-104.8\left(\mathrm{~d},{ }^{2} J=69.1 \mathrm{~Hz}, 1 \mathrm{~F}\right) \mathrm{ppm} ; \bar{v} /($ neat $)=3430$, 2926, 1736, 1666, 1426, 1158, 1056, $989 \mathrm{~cm}^{-1}$; HRMS (NSI-ES): calcd for $\mathrm{C}_{19} \mathrm{H}_{32} \mathrm{~F}_{2} \mathrm{NO}_{6}$, 408.2192 [M+H] $]^{+}$, found: 408.2189; MS (CI): $m / z(\%): 408(100)[\mathrm{M}+\mathrm{H}]^{+} ; \mathrm{t}_{\mathrm{R}}(\mathrm{GC})=14.91$ minutes. ${ }^{* *}$ *this is by comparison with compounds from the Saegusa-Ito series. ${ }^{5 * *}$ the cisand trans-stereoisomers appeared as one peak by GC.

\section{1,1-difluoro-2-((2-methoxyethoxy)methoxy)-3-methylhepta-1,6-dien-3-ol}

$(3 e)$.

Prepared as for trans-3a and cis-3a from acetal (1.90 mL, $12 \mathrm{mmol}), n$-butyllithium $(12.9 \mathrm{~mL}$ of a $1.94 \mathrm{M}$ solution in hexanes, $25 \mathrm{mmol})$, diisopropylamine $(3.70 \mathrm{~mL}, 26 \mathrm{mmol})$ and 5 hexen-2-one $(1.39 \mathrm{~mL}, 12 \mathrm{mmol})$ in THF $(12 \mathrm{~mL})$. The crude product $(2.54 \mathrm{~g})$ was purified by Kugelrohr distillation to afford 3e $(2.33 \mathrm{~g}, 73 \%)$ as a pale yellow oil. b.p. $=78{ }^{\circ} \mathrm{C} / 0.04$ $\mathrm{mmHg} ; \mathrm{R}_{\mathrm{f}}=0.27$ (50\% diethyl ether in hexane); ${ }^{1} \mathrm{H}$ NMR $\left(400 \mathrm{MHz}, \mathrm{CDCl}_{3}\right): \delta=5.85$ (ddt, $J=16.9,10.1,6.5 \mathrm{~Hz}, 1 \mathrm{H}), 5.06\left(\mathrm{dq}, J=16.9,{ }^{4} J={ }^{2} J=1.9 \mathrm{~Hz}, 1 \mathrm{H}\right), 5.00\left(\mathrm{~d},{ }^{2} J=6.4 \mathrm{~Hz}\right.$, $1 \mathrm{H}), 4.97\left(\mathrm{dq}, J=10.1,{ }^{4} J={ }^{2} J=1.9 \mathrm{~Hz}, 1 \mathrm{H}\right), 4.93\left(\mathrm{~d},{ }^{2} J=6.4 \mathrm{~Hz}, 1 \mathrm{H}\right), 3.98-3.81(\mathrm{~m}, 2 \mathrm{H})$, 3.59 (br. t, $J=4.8 \mathrm{~Hz}, 2 \mathrm{H}$ ), 3.41 (s, 3H), 3.39 (br. s, $1 \mathrm{H}$ ), 2.10 (app. qt, $J=7.6,{ }^{4} J=1.9 \mathrm{~Hz}$, $2 \mathrm{H}), 1.87-1.68(\mathrm{~m}, 2 \mathrm{H}), 1.42\left(\mathrm{~d},{ }^{5} \mathrm{~J}_{\mathrm{H}-\mathrm{F}}=4.8 \mathrm{~Hz}, 3 \mathrm{H}\right) \mathrm{ppm} ;{ }^{13} \mathrm{C}\left(100 \mathrm{MHz}, \mathrm{CDCl}_{3}\right): \delta=154.2$ $\left(\mathrm{t},{ }^{1} J_{\mathrm{C}-\mathrm{F}}=288.6 \mathrm{~Hz}\right), 137.8,121.0\left(\mathrm{dd},{ }^{2} J_{\mathrm{C}-\mathrm{F}}=32.7,10.5 \mathrm{~Hz}\right), 114.0,98.5\left(\mathrm{t},{ }^{4} J_{\mathrm{C}-\mathrm{F}}=4.1 \mathrm{~Hz}\right)$, $71.1\left(\mathrm{~d},{ }^{3} J_{\mathrm{C}-\mathrm{F}}=3.7 \mathrm{~Hz}\right), 71.0,68.5,58.5,39.3,28.1,24.5\left(\mathrm{~d},{ }^{4} J_{\mathrm{C}-\mathrm{F}}=7.1 \mathrm{~Hz}\right) \mathrm{ppm} ;{ }^{19} \mathrm{~F}(376$ $\left.\mathrm{MHz}, \mathrm{CDCl}_{3}\right): \delta=-98.4\left(\mathrm{~d},{ }^{2} J=70.9 \mathrm{~Hz}, 1 \mathrm{~F}\right),-103.9\left(\right.$ app. dq, ${ }^{2} J=70.9 \mathrm{~Hz},{ }^{5} J_{\mathrm{F}-\mathrm{H}}=4.8 \mathrm{~Hz}$ 1F) $\mathrm{ppm} ; \overline{\mathrm{v}} /($ neat $)=3447,2928,1736,1102,1024 \mathrm{~cm}^{-1}$; HRMS (NSI-ES): calcd for $\mathrm{C}_{12} \mathrm{H}_{20} \mathrm{~F}_{2} \mathrm{O}_{4} \mathrm{Na}, 289.1222[\mathrm{M}+\mathrm{Na}]^{+}$, found: 289.1221; MS (CI): $m / z$ (\%): 161 (4) [M$\left.\mathrm{C}_{4} \mathrm{H}_{9} \mathrm{O}_{3}\right]^{+}, 89(73)\left[\mathrm{C}_{4} \mathrm{H}_{9} \mathrm{O}_{2}\right]^{+}, 59(100)\left[\mathrm{C}_{3} \mathrm{H}_{7} \mathrm{O}\right]^{+} ; \mathrm{t}_{\mathrm{R}}(\mathrm{GC})=11.38$ minutes.

\section{1,1-Difluoro-2-((methoxyethoxy)methoxy)-3-methyl-4-cyclopropyl-hepta-1,6-dien-3-ol}

(3h). Prepared as for trans-3a and cis-3a from acetal (1.26 mL, $8 \mathrm{mmol}), n$-butyllithium (8.3 
$\mathrm{mL}$ of a $1.98 \mathrm{M}$ solution in hexanes, $16 \mathrm{mmol})$, diisopropylamine $(2.38 \mathrm{~mL}, 17 \mathrm{mmol})$ and 1 (1-allylcyclopropyl)ethan-1-one ${ }^{5}(1.00 \mathrm{~g}, 8 \mathrm{mmol})$ in THF $(8 \mathrm{~mL})$. The crude product $(1.41$ g) was purified by Kugelrohr distillation to afford $\mathbf{3 h}(0.730 \mathrm{~g}, 31 \%)$ as a pale yellow oil. b.p. $=93{ }^{\circ} \mathrm{C} / 0.06 \mathrm{mmHg} ; \mathrm{R}_{\mathrm{f}}=0.54$ (40\% ethyl acetate in hexane); ${ }^{1} \mathrm{H}$ NMR (400 MHz, $\left.\mathrm{CDCl}_{3}\right): \delta=5.70(\mathrm{ddt}, J=17.3,10.2,7.3 \mathrm{~Hz}, 1 \mathrm{H}), 5.11-4.96\left(\mathrm{~m}\right.$, including $5.05\left(\mathrm{~d},{ }^{2} J=6.4\right.$ $\mathrm{Hz}, 1 \mathrm{H}), 2 \mathrm{H}), 4.91\left(\mathrm{~d},{ }^{2} J=6.4 \mathrm{~Hz}, 1 \mathrm{H}\right), 4.00-3.80(\mathrm{~m}, 2 \mathrm{H}), 3.59$ (t, $\left.J=4.8 \mathrm{~Hz}, 2 \mathrm{H}\right), 3.41$ (s, 3H), 3.12 (br. s, 1H), 2.34-2.13 (m, 2H), $1.42\left(\mathrm{~d},{ }^{5} J_{\mathrm{H}-\mathrm{F}}=5.8 \mathrm{~Hz}, 3 \mathrm{H}\right), 0.84-0.75(\mathrm{~m}, 1 \mathrm{H}), 0.63$ $\left(\mathrm{ddd},{ }^{2} J=9.9, J=5.8,4.3 \mathrm{~Hz}, 1 \mathrm{H}\right) 0.45-0.35(\mathrm{~m}, 1 \mathrm{H}), 0.29\left(\mathrm{ddd},{ }^{2} J=9.9, J=5.5,4.3 \mathrm{~Hz}\right.$, 1H) ppm; ${ }^{13} \mathrm{C}\left(100 \mathrm{MHz}, \mathrm{CDCl}_{3}\right): \delta=154.6\left(\mathrm{t},{ }^{1} J_{\mathrm{C}-\mathrm{F}}=288.8 \mathrm{~Hz}\right), 135.6,120.3\left(\mathrm{dd},{ }^{2} J_{\mathrm{C}-\mathrm{F}}=\right.$ $32.6,11.5 \mathrm{~Hz}), 116.0,98.4\left(\mathrm{t},{ }^{4} J_{\mathrm{C}-\mathrm{F}}=4.2 \mathrm{~Hz}\right), 72.1\left(\mathrm{~d},{ }^{3} J_{\mathrm{C}-\mathrm{F}}=6.3 \mathrm{~Hz}\right), 71.1,68.6,58.5,37.1$, 25.6, $22.9\left(\mathrm{~d},{ }^{4} J_{\mathrm{C}-\mathrm{F}}=8.5 \mathrm{~Hz}\right), 7.0,5.1 \mathrm{ppm} ;{ }^{19} \mathrm{~F}\left(376 \mathrm{MHz}, \mathrm{CDCl}_{3}\right): \delta=-97.7\left(\mathrm{~d},{ }^{2} J=68.4\right.$ $\mathrm{Hz}, 1 \mathrm{~F}),-102.7\left(\mathrm{dq},{ }^{2} J=68.4 \mathrm{~Hz},{ }^{5} J_{\mathrm{F}-\mathrm{H}}=5.8 \mathrm{~Hz}, 1 \mathrm{~F}\right) \mathrm{ppm} ; \bar{v} /($ neat $)=3465,2887,1736$, 1022, $937 \mathrm{~cm}^{-1}$; HRMS (ESI): calcd for $\mathrm{C}_{14} \mathrm{H}_{26} \mathrm{~F}_{2} \mathrm{O}_{4} \mathrm{~N}, 310.1824\left[\mathrm{M}+\mathrm{NH}_{4}\right]^{+}$, found: 310.1826 ;

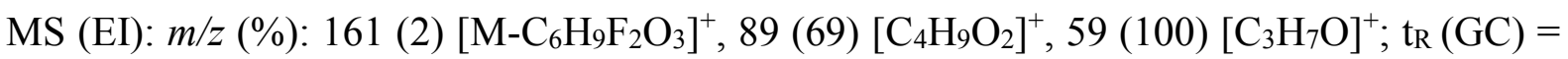
12.75 minutes.

\section{1,1-Difluoro-2-((methoxyethoxy)methoxy)-3-methyl-4-cyclopentyl-hepta-1,6-dien-3-ol}

(3i). Prepared as for trans-3a and cis-3a from acetal (1.58 mL, $10 \mathrm{mmol}), n$-butyllithium $(9.8$ $\mathrm{mL}$ of a $2.04 \mathrm{M}$ solution in hexanes, $20 \mathrm{mmol})$, diisopropylamine $(3.38 \mathrm{~mL}, 24 \mathrm{mmol})$ and 1 (1-allylcyclopentyl)ethan-1-one $(1.52 \mathrm{~g}, 10 \mathrm{mmol})$ in THF $(11 \mathrm{~mL})$. The crude product $(2.13$ g) was purified by Kugelrohr distillation to afford $3 \mathbf{i}(0.965 \mathrm{~g}, 30 \%)$ as a pale yellow oil. b.p. $=100{ }^{\circ} \mathrm{C} / 0.04 \mathrm{mmHg} ; \mathrm{R}_{\mathrm{f}}=0.30$ (40\% diethyl ether in hexane); ${ }^{1} \mathrm{H}$ NMR $(400 \mathrm{MHz}$, $\left.\mathrm{CDCl}_{3}\right): \delta=5.70(\mathrm{ddt}, J=17.3,10.1,7.3 \mathrm{~Hz}, 1 \mathrm{H}), 5.11-4.99\left(\mathrm{~m}\right.$, including $5.04\left(\mathrm{~d},{ }^{2} J=6.3\right.$ $\mathrm{Hz}, 1 \mathrm{H}), 2 \mathrm{H}), 4.88\left(\mathrm{dt},{ }^{2} J=6.4,{ }^{5} J_{\mathrm{H}-\mathrm{F}}=1 \mathrm{~Hz}, 1 \mathrm{H}\right), 3.94-3.80(\mathrm{~m}, 2 \mathrm{H}), 3.58(\mathrm{t}, J=4.8 \mathrm{~Hz}$, 2H), 3.40 (s, 3H), 3.12 (br. s, 1H), 2.19 (d, J=7.3 Hz, 2H), 1.88-1.74 (m, 2H), 1.68-1.35 (m, including $\left.1.41\left(\mathrm{~d},{ }^{5} J_{\mathrm{H}-\mathrm{F}}=5.4 \mathrm{~Hz}, 3 \mathrm{H}\right), 6 \mathrm{H}\right) \mathrm{ppm} ;{ }^{13} \mathrm{C}\left(100 \mathrm{MHz}, \mathrm{CDCl}_{3}\right): \delta=155.0\left(\mathrm{t},{ }^{1} J_{\mathrm{C}-\mathrm{F}}=\right.$ 
$288.3 \mathrm{~Hz}), 136.2,120.3\left(\mathrm{dd},{ }^{2} J_{\mathrm{C}-\mathrm{F}}=32.1,11.3 \mathrm{~Hz}\right), 116.3,99.0\left(\mathrm{t},{ }^{4} J_{\mathrm{C}-\mathrm{F}}=4.7 \mathrm{~Hz}\right), 75.9(\mathrm{~d}$, $\left.{ }^{3} J_{\mathrm{C}-\mathrm{F}}=4.8 \mathrm{~Hz}\right), 71.1,68.9,58.5,54.1,41.1,32.1,31.6,25.9,25.2,22.3\left(\mathrm{~d},{ }^{4} J_{\mathrm{C}-\mathrm{F}}=8.6 \mathrm{~Hz}\right)$ ppm; ${ }^{19} \mathrm{~F}\left(376 \mathrm{MHz}, \mathrm{CDCl}_{3}\right): \delta=-97.7\left(\mathrm{~d},{ }^{2} J=71.0 \mathrm{~Hz}, 1 \mathrm{~F}\right),-101.33\left(\mathrm{dq},{ }^{2} J=71.0 \mathrm{~Hz},{ }^{5} J_{\mathrm{F}-\mathrm{H}}\right.$ $=5.4 \mathrm{~Hz}, 1 \mathrm{~F}) \mathrm{ppm}$; the $1.0 \mathrm{~Hz}{ }^{5} J_{\mathrm{H}-\mathrm{F}}=$ splitting could not be resolved in the $376 \mathrm{MHz}{ }^{19} \mathrm{~F}$ NMR spectrum; $\bar{v} /($ neat $)=3494,2944,1731,1266,1065,909 \mathrm{~cm}^{-1}$; HRMS (APCI): calcd for $\mathrm{C}_{16} \mathrm{H}_{30} \mathrm{~F}_{2} \mathrm{O}_{4} \mathrm{~N}, 338.2137\left[\mathrm{M}+\mathrm{NH}_{4}\right]^{+}$, found: 338.2143; $\mathrm{MS}$ (CI): $\mathrm{m} / \mathrm{z}$ (\%): 338 (100) $\left[\mathrm{M}+\mathrm{NH}_{4}\right]^{+} ; \mathrm{t}_{\mathrm{R}}(\mathrm{GC})=13.78$ minutes.

Trans-1-(2,2-difluoro-1-((2-methoxyethoxy)methoxy) vinyl)-2-(prop-2-yn-1-yl)cyclohexan1-ol (trans-3j) and cis-1-(2,2-difluoro-1-((2-methoxyethoxy)methoxy)vinyl)-2-(prop-2-yn-1yl)cyclohexan-1-ol (cis-3j). Prepared as for trans-3a and cis-3a from acetal (0.79 mL, $5 \mathrm{mmol})$, $n$-butyllithium $(5.4 \mathrm{~mL}$ of a $1.84 \mathrm{M}$ solution in hexanes, $10 \mathrm{mmol})$, diisopropylamine $(1.69$ $\mathrm{mL}, 12 \mathrm{mmol})$ and 2-(prop-2-ynyl)cyclohexanone ${ }^{23}(0.68 \mathrm{~g}, 5 \mathrm{mmol})$ in THF $(5 \mathrm{~mL})$. The crude allylic alcohol was purified by flash column chromatography using a Thompson Single Step cartridge (90 g cartridge, $20 \%$ ethyl acetate in hexane) to afford an inseparable mixture of trans-3j and cis-3j as a pale orange oil $(0.291 \mathrm{~g}, 19 \%, 97: 3) . \mathrm{R}_{\mathrm{f}}=0.82(50 \%$ ethyl acetate in hexane); ${ }^{1} \mathrm{H}$ NMR (400 MHz, $\mathrm{CDCl}_{3}$ ): $\delta=4.94$ (app. s, 2H), 3.94-3.81 (m, 2H), 3.59 (app. t, $J=4.8 \mathrm{~Hz}, 2 \mathrm{H}), 3.41(\mathrm{~s}, 3 \mathrm{H}), 2.93(\mathrm{~s}, 1 \mathrm{H}), 2.34\left(\mathrm{dt},{ }^{2} J=16.9, J={ }^{4} J=2.8 \mathrm{~Hz}, 1 \mathrm{H}\right), 2.20$ (ddd, $\left.{ }^{2} J=16.9, J=8.9,{ }^{4} J=2.8 \mathrm{~Hz}, 1 \mathrm{H}\right), 1.99\left(\mathrm{t},{ }^{4} J=2.8 \mathrm{~Hz}, 1 \mathrm{H}\right), 1.94-1.47$ (envelope, $\left.8 \mathrm{H}\right)$, 1.36-1.21 (m, 1H) ppm; ${ }^{13} \mathrm{C} \mathrm{NMR}\left(100 \mathrm{MHz}, \mathrm{CDCl}_{3}\right): \delta=154.0\left(\mathrm{t},{ }^{1} J_{\mathrm{C}-\mathrm{F}}=289.2 \mathrm{~Hz}\right), 120.8$ $\left(\mathrm{dd},{ }^{2} J_{\mathrm{C}-\mathrm{F}}=32.9 .10 .0 \mathrm{~Hz}\right), 98.3\left(\mathrm{t},{ }^{4} J_{\mathrm{C}-\mathrm{F}}=4.5 \mathrm{~Hz}\right), 83.3,72.6\left(\mathrm{~d},{ }^{3} J_{\mathrm{C}-\mathrm{F}}=5.6 \mathrm{~Hz}\right), 71.1,68.8$, 68.5, 58.5, $41.1\left(\mathrm{~d},{ }^{4} J_{\mathrm{C}-\mathrm{F}}=4.7 \mathrm{~Hz}\right), 36.4\left(\mathrm{t},{ }^{4} J_{\mathrm{C}-\mathrm{F}}=3.1 \mathrm{~Hz}\right), 26.1,24.7,20.6,19.7 \mathrm{ppm}$; Major trans-diastereoisomer $\mathbf{3 j}$ (assigned on the basis of $\delta$ and intensity) ${ }^{19} \mathrm{~F}\left(376 \mathrm{MHz}, \mathrm{CDCl}_{3}\right): \delta$ $=-98.0\left(\mathrm{~d},{ }^{2} J=72.3 \mathrm{~Hz}, 1 \mathrm{~F}\right),-103.1\left(\mathrm{~d},{ }^{2} J=72.3 \mathrm{~Hz}, 1 \mathrm{~F}\right) \mathrm{ppm} ; *$ Minor cis-diastereoisomer $\mathbf{3 j}$ (assigned on the basis of $\delta$ and intensity ${ }^{19} \mathrm{~F}\left(376 \mathrm{MHz}, \mathrm{CDCl}_{3}\right): \delta=-96.7\left(\mathrm{~d},{ }^{2} \mathrm{~J}=67.2 \mathrm{~Hz}\right.$, 1F), $-103.7\left(\mathrm{~d},{ }^{2} J=67.2 \mathrm{~Hz}, 1 \mathrm{~F}\right) \mathrm{ppm} ; \bar{v} /($ neat $)=3452,3302,2928,1734,1216,1056 \mathrm{~cm}^{-1}$; 
HRMS (ESI): calcd for $\mathrm{C}_{15} \mathrm{H}_{23} \mathrm{~F}_{2} \mathrm{O}_{4}, 305.1564[\mathrm{M}+\mathrm{H}]^{+}$, found: 305.1557 ; MS (CI): $m / z(\%)$ : $305(10)[\mathrm{M}+\mathrm{H}]^{+}, 322(15)\left[\mathrm{M}+\mathrm{NH}_{4}\right]^{+} ; \mathrm{t}_{\mathrm{R}}(\mathrm{GC})=12.90$ minutes. $* * *$ this is by comparison with compounds from the Saegusa-Ito series. ${ }^{5 * *}$ the $c i s$ - and trans-stereoisomers appeared as one peak by GC.

1,1-difluoro-2-((2-methoxyethoxy)methoxy)non-1-en-3-ol (3k). Prepared as for trans3a and cis-3a from acetal (1.90 mL, $12 \mathrm{mmol}), n$-butyllithium (12.9 $\mathrm{mL}$ of a $1.94 \mathrm{M}$ solution in hexanes, $25 \mathrm{mmol})$, diisopropylamine $(3.70 \mathrm{~mL}, 26 \mathrm{mmol})$ and heptanal $(1.37 \mathrm{~g}, 12 \mathrm{mmol})$ in THF (12 mL). The crude allylic alcohol (2.63 g) was purified by Kugelrohr distillation to afford 3k as a pale yellow oil $(2.20 \mathrm{~g}, 65 \%)$. b.p. $=102{ }^{\circ} \mathrm{C} / 0.05 \mathrm{mmHg} ; \mathrm{R}_{\mathrm{f}}=0.21(50 \%$ diethyl ether in hexane); ${ }^{1} \mathrm{H}$ NMR (400 MHz, $\left.\mathrm{CDCl}_{3}\right): \delta=5.00\left(\mathrm{~d},{ }^{2} J=6.8 \mathrm{~Hz}, 1 \mathrm{H}\right), 4.88(\mathrm{~d}$, $\left.{ }^{2} J=6.8 \mathrm{~Hz}, 1 \mathrm{H}\right), 4.24\left(\mathrm{dtdd}, J=9.3, J=7.4,{ }^{4} J_{\mathrm{H}-\mathrm{F}}=3.6,2.0 \mathrm{~Hz}, 1 \mathrm{H}\right), 3.96\left(\mathrm{ddd},{ }^{2} J=10.8, J\right.$ $=6.4,3.5 \mathrm{~Hz}, 1 \mathrm{H}), 3.78\left(\mathrm{ddd},{ }^{2} J=10.8, J=5.0,3.1 \mathrm{~Hz}, 1 \mathrm{H}\right), 3.66-3.53(\mathrm{~m}, 2 \mathrm{H}), 3.40(\mathrm{~s}$, $3 \mathrm{H},), 3.20(\mathrm{~d}, J=9.3 \mathrm{~Hz}, 1 \mathrm{H}), 1.79-1.52(\mathrm{~m}, 2 \mathrm{H}), 1.41-1.20(\mathrm{~m}, 8 \mathrm{H}), 0.88(\mathrm{t}, J=6.9 \mathrm{~Hz}, 3 \mathrm{H})$ ppm; ${ }^{13} \mathrm{C} \mathrm{NMR}\left(100 \mathrm{MHz}, \mathrm{CDCl}_{3}\right): \delta=154.7\left(\mathrm{dd},{ }^{1} J_{\mathrm{C}-\mathrm{F}}=291.6,284.7 \mathrm{~Hz}\right), 118.2\left(\mathrm{dd},{ }^{2} J_{\mathrm{C}-\mathrm{F}}=\right.$ 36.3. $9.8 \mathrm{~Hz}), 98.0,71.4,68.4,67.1,58.9,33.9,31.7,29.0,25.4,22.5,13.9 \mathrm{ppm} ;{ }^{19} \mathrm{~F}(376$ $\left.\mathrm{MHz}, \mathrm{CDCl}_{3}\right): \delta=-100.5\left(\mathrm{~d},{ }^{2} J=63.7 \mathrm{~Hz}, 1 \mathrm{~F}\right),-110.1\left(\mathrm{~d},{ }^{2} J=63.7 \mathrm{~Hz}, 1 \mathrm{~F}\right) \mathrm{ppm} ;\left(\right.$ the ${ }^{19} \mathrm{~F}-{ }^{1} \mathrm{H}$ splitting was not resolved in the ${ }^{19} \mathrm{~F}$ NMR spectrum); $\bar{v} /($ neat $)=3421,2924,1749,1232$, 1054, $955 \mathrm{~cm}^{-1}$; HRMS (ESI): calcd for $\mathrm{C}_{13} \mathrm{H}_{24} \mathrm{~F}_{2} \mathrm{O}_{4} \mathrm{Na}, 305.1535[\mathrm{M}+\mathrm{Na}]^{+}$, found: 305.1532 ; MS (CI): $m / z(\%): 283(1)[\mathrm{M}+\mathrm{H}]^{+}, 265(2)[\mathrm{M}-\mathrm{OH}]^{+}, 89(78)\left[\mathrm{C}_{4} \mathrm{H}_{9} \mathrm{O}_{2}\right]^{+}, 59(100)\left[\mathrm{C}_{3} \mathrm{H}_{7} \mathrm{O}\right]^{+}$; $t_{R}(\mathrm{GC})=12.41$ minutes.

1,1 Difuoro-2-((2-methoxyethoxy)methoxy) 3-cyclohexyl prop-2-en-3-ol (3l) Prepared as for trans-3a and cis-3a from acetal (1.90 mL, $12 \mathrm{mmol}), n$-butyllithium (11.9 mL of a 2.10 M solution in hexanes, $25 \mathrm{mmol})$, diisopropylamine $(3.70 \mathrm{~mL}, 26 \mathrm{mmol})$ and cyclohexanecarboxaldehyde $(1.34 \mathrm{~g}, 12 \mathrm{mmol})$ in THF $(12 \mathrm{~mL})$. The crude allylic alcohol 
(3.12 g) was purified by Kugelrohr distillation to afford $3 \mathbf{l}$ as a pale yellow oil (2.60 g, 77 \%). b.p. $=102{ }^{\circ} \mathrm{C} / 0.06 \mathrm{mmHg} ; \mathrm{R}_{\mathrm{f}}=0.3$ (50\% diethyl ether in hexane); ${ }^{1} \mathrm{H}$ NMR (400 MHz, $\left.\mathrm{CDCl}_{3}\right): \delta=5.01\left(\mathrm{~d},{ }^{2} J=6.8 \mathrm{~Hz}, 1 \mathrm{H}\right), 4.87\left(\mathrm{~d},{ }^{2} J=6.8 \mathrm{~Hz}, 1 \mathrm{H}\right), 3.96\left(\mathrm{ddd},{ }^{2} J=10.8, J=6.0\right.$, $3.1 \mathrm{~Hz}, 1 \mathrm{H}), 3.87$ (br. d, $J=8.3 \mathrm{~Hz}, 1 \mathrm{H}), 3.78\left(\mathrm{ddd},{ }^{2} J=10.8, J=4.8,3.0 \mathrm{~Hz}, 1 \mathrm{H}\right), 3.65-3.53$ (m, 2H), $3.40(\mathrm{~s}, 3 \mathrm{H}), 3.18$ (br. s, $1 \mathrm{H}), 2.10$ (br. d, $\left.{ }^{2} J=13.7 \mathrm{~Hz}, 1 \mathrm{H}\right), 1.86-1.52(\mathrm{~m}, 5 \mathrm{H})$, 1.34-1.10 (m, 3H), 1.08-0.94 (m, 1H), 0.94-0.79 (m, 1H) ppm; $\left.{ }^{13} \mathrm{C} \mathrm{NMR} \mathrm{(100} \mathrm{MHz,} \mathrm{CDCl}_{3}\right)$ : $\delta=154.6\left(\mathrm{dd},{ }^{1} J_{\mathrm{C}-\mathrm{F}}=292.2,285.2 \mathrm{~Hz}\right), 117.0\left(\mathrm{dd},{ }^{2} J_{\mathrm{C}-\mathrm{F}}=37.7 .10 .0 \mathrm{~Hz}\right), 97.5\left(\mathrm{t},{ }^{4} J_{\mathrm{C}-\mathrm{F}}=3.6\right.$ $\mathrm{Hz}), 71.2,70.9,68.0,58.4,40.2,29.0,28.3,25.9,25.3,25.2 \mathrm{ppm} ;{ }^{19} \mathrm{~F}\left(376 \mathrm{MHz}, \mathrm{CDCl}_{3}\right): \delta$ $=-100.6\left(\mathrm{~d},{ }^{2} J=65.5 \mathrm{~Hz}, 1 \mathrm{~F}\right),-110.6\left(\mathrm{~d},{ }^{2} J=65.5 \mathrm{~Hz}, 1 \mathrm{~F}\right) \mathrm{ppm} ; \bar{v} /($ neat $)=3463,2921,1749$, 1232, 1013, $953 \mathrm{~cm}^{-1}$; HRMS (APCI): calcd for $\mathrm{C}_{13} \mathrm{H}_{26} \mathrm{~F}_{2} \mathrm{O}_{4} \mathrm{~N}_{1}, 298.1824\left[\mathrm{M}+\mathrm{NH}_{4}\right]^{+}$, found: 298.1819; MS (EI): $m / z(\%): 204(2)\left[\mathrm{M}-\mathrm{C}_{4} \mathrm{H}_{9} \mathrm{~F}\right]^{+}, 89(100)\left[\mathrm{C}_{4} \mathrm{H}_{9} \mathrm{O}_{2}\right]^{+}, 59(100)\left[\mathrm{C}_{3} \mathrm{H}_{7} \mathrm{O}\right]^{+}$; $\mathrm{t}_{\mathrm{R}}(\mathrm{GC})=12.65$ minutes.

\section{1,1-difluoro-2-((2-methoxyethoxy)methoxy)-3-(tetrahydro-2H-pyran-4-yl)prop-2-en-}

3-ol (3m). Prepared as for trans-3a and cis-3a from acetal (1.90 mL, $12 \mathrm{mmol}), n$ butyllithium (11.9 $\mathrm{mL}$ of a $2.10 \mathrm{M}$ solution in hexanes, $25 \mathrm{mmol})$, diisopropylamine $(3.70$ $\mathrm{mL}, 26 \mathrm{mmol})$ and 4-formyltetrahydropyran (1.37 g, $12 \mathrm{mmol})$ in THF (12 mL). The crude allylic alcohol $(2.71 \mathrm{~g})$ was purified by flash column chromatography (90 g cartridge, $90 \%$ diethyl ether in hexane) to afford $\mathbf{3 m}$ as a pale yellow oil (1.94 g, $57 \%)$. $\mathrm{R}_{\mathrm{f}}=0.30(90 \%$ diethyl ether in hexane); ${ }^{1} \mathrm{H}$ NMR $\left(400 \mathrm{MHz}, \mathrm{CDCl}_{3}\right): \delta=5.00(\mathrm{~d}, J=6.6 \mathrm{~Hz}, 1 \mathrm{H}), 4.88(\mathrm{~d}$, $J=6.6 \mathrm{~Hz}, 1 \mathrm{H}), 4.09-3.84(\mathrm{~m}, 4 \mathrm{H}), 3.77\left(\mathrm{ddd},{ }^{2} J=10.8, J=4.5,3.1 \mathrm{~Hz}, 1 \mathrm{H}\right), 3.65-3.52(\mathrm{~m}$, 2H), 3.45-3.30 (m, 6H), 2.03-1.92 (m, 1H), 1.91-1.75 (m, 1H), 1.52-1.31 (m, 2H), $1.23(\mathrm{qd}, J$ $=11.9,4.4 \mathrm{~Hz}, 1 \mathrm{H}) \mathrm{ppm} ;{ }^{13} \mathrm{C} \mathrm{NMR}\left(100 \mathrm{MHz}, \mathrm{CDCl}_{3}\right): \delta=154.7\left(\mathrm{dd},{ }^{1} J_{\mathrm{C}-\mathrm{F}}=292.8,285.6\right.$ $\mathrm{Hz}), 116.5\left(\mathrm{dd},{ }^{2} J_{\mathrm{C}-\mathrm{F}}=37.2,11.0 \mathrm{~Hz}\right), 97.4\left(\mathrm{t},{ }^{4} J_{\mathrm{C}-\mathrm{F}}=3.8 \mathrm{~Hz}\right), 70.9,70.8\left(\mathrm{t},{ }^{3} J_{\mathrm{C}-\mathrm{F}}=3.0 \mathrm{~Hz}\right)$, 68.0, 67.2, 66.9, 58.5, 37.6, 29.3, $\left.28.2 \mathrm{ppm} ;{ }^{19} \mathrm{~F} \mathrm{NMR} \mathrm{(376} \mathrm{MHz,} \mathrm{CDCl}_{3}\right): \delta=-99.7\left(\mathrm{~d},{ }^{2} J=\right.$ 
$64.0 \mathrm{~Hz}, 1 \mathrm{~F}),-109.9\left(\mathrm{~d},{ }^{2} J=64.0 \mathrm{~Hz}, 1 \mathrm{~F}\right) \mathrm{ppm} ; \bar{v} /($ neat $)=3404,2915,1749,1230,1026,955$ $\mathrm{cm}^{-1}$; HRMS (ESI): calcd for $\mathrm{C}_{12} \mathrm{H}_{24} \mathrm{~F}_{2} \mathrm{O}_{5} \mathrm{~N}_{1}, 300.1617$ [M+NH$]_{4}^{+}$, found: 300.1620; MS (EI): $m / z(\%): 281(1)[\mathrm{M}-\mathrm{H}]^{+}, 89(92)\left[\mathrm{C}_{4} \mathrm{H}_{9} \mathrm{O}_{2}\right]^{+}, 59(100)\left[\mathrm{C}_{3} \mathrm{H}_{7} \mathrm{O}\right]^{+} ; \mathrm{t}_{\mathrm{R}}(\mathrm{GC})=12.88$ minutes.

1,1-difluoro-2-((2-methoxyethoxy)methoxy)-3-(4-(trifluoromethyl)phenyl)prop-2-en3-ol (3o). Prepared as for trans-3a and cis-3a from acetal (1.90 mL, $12 \mathrm{mmol}), n$-butyllithium (11.9 $\mathrm{mL}$ of a $2.10 \mathrm{M}$ solution in hexanes, $25 \mathrm{mmol})$, diisopropylamine $(3.70 \mathrm{~mL}, 26 \mathrm{mmol})$ and $p$-trifluoromethylbenzaldehyde $(2.09 \mathrm{~g}, 12 \mathrm{mmol})$ in THF $(12 \mathrm{~mL})$. The crude allylic alcohol (4.99 g) was purified by Kugelrohr distillation followed by flash column chromatography (90 g cartridge, $60 \%$ diethyl ether in petroleum ether) to afford 3o as a colourless oil $(0.87 \mathrm{~g}, 21 \%)$. b.p. $=113{ }^{\circ} \mathrm{C} / 0.05 \mathrm{mmHg} ; \mathrm{R}_{\mathrm{f}}=0.62(5 \%$ acetone in dichloromethane); ${ }^{1} \mathrm{H}$ NMR $\left(400 \mathrm{MHz}, \mathrm{CDCl}_{3}\right): \delta=7.64(\mathrm{~d}, J=8.4 \mathrm{~Hz}, 2 \mathrm{H}), 7.57(\mathrm{~d}, J=8.4$ $\mathrm{Hz}, 2 \mathrm{H}), 5.53$ (br. d, $J=8.7 \mathrm{~Hz}, 1 \mathrm{H}), 4.93\left(\mathrm{~d},{ }^{2} J=6.7 \mathrm{~Hz}, 1 \mathrm{H}\right), 4.87\left(\mathrm{~d},{ }^{2} J=6.7 \mathrm{~Hz}, 1 \mathrm{H}\right)$, $3.85(\mathrm{~d}, J=8.7 \mathrm{~Hz}, 1 \mathrm{H}), 3.76\left(\mathrm{ddd},{ }^{2} J=10.8, J=5.9,3.8 \mathrm{~Hz}, 1 \mathrm{H}\right), 3.64\left(\mathrm{ddd},{ }^{2} J=10.8, J=\right.$ 4.9, $3.1 \mathrm{~Hz}, 1 \mathrm{H}), 3.58-3.48(\mathrm{~m}, 2 \mathrm{H}), 3.39(\mathrm{~s}, 3 \mathrm{H}) \mathrm{ppm} ;{ }^{13} \mathrm{C} \mathrm{NMR}\left(100 \mathrm{MHz}, \mathrm{CDCl}_{3}\right): \delta=$ $154.5\left(\mathrm{dd},{ }^{1} J_{\mathrm{C}-\mathrm{F}}=293.4,287.4 \mathrm{~Hz}\right), 143.9,129.3\left(\mathrm{q},{ }^{2} J_{\mathrm{C}-\mathrm{F}}=32.3 \mathrm{~Hz}\right), 125.8,124.7\left(\mathrm{q},{ }^{3} J_{\mathrm{C}-\mathrm{F}}=\right.$ $3.5 \mathrm{~Hz}), 123.6\left(\mathrm{q},{ }^{1} J_{\mathrm{C}-\mathrm{F}}=271.8 \mathrm{~Hz}\right), 117.3\left(\mathrm{dd},{ }^{2} J_{\mathrm{C}-\mathrm{F}}=36.0,11.3 \mathrm{~Hz}\right), 97.3\left(\mathrm{t},{ }^{4} J_{\mathrm{C}-\mathrm{F}}=3.9 \mathrm{~Hz}\right)$, 70.7, 68.0, $67.5\left(\mathrm{~d},{ }^{3} J_{\mathrm{C}-\mathrm{F}}=3.4 \mathrm{~Hz}\right), 58.4 \mathrm{ppm} ;{ }^{19} \mathrm{~F} \mathrm{NMR}\left(376 \mathrm{MHz}, \mathrm{CDCl}_{3}\right): \delta=-62.5(\mathrm{~s}, 3 \mathrm{~F})$, $-98.5\left(\mathrm{dd},{ }^{2} J=60.1,{ }^{4} J_{\mathrm{F}-\mathrm{H}}=2.0 \mathrm{~Hz}, 1 \mathrm{~F}\right),-108.5\left(\mathrm{dd},{ }^{2} J=60.1,{ }^{4} J_{\mathrm{F}-\mathrm{H}}=3.2 \mathrm{~Hz}, 1 \mathrm{~F}\right) \mathrm{ppm}$; $\bar{v} /($ neat $)=3419,2930,1751,1325,1112,1067,1017 \mathrm{~cm}^{-1}$; HRMS (APCI): calcd for $\mathrm{C}_{14} \mathrm{H}_{19} \mathrm{~F}_{5} \mathrm{O}_{4} \mathrm{~N}_{1}, 360.1229\left[\mathrm{M}+\mathrm{NH}_{4}\right]^{+}$, found: $360.1231 ; \mathrm{MS}$ (EI): $m / z(\%): 323$ (1) [M-F] $]^{+}, 175$ (9) $\left[\mathrm{C}_{8} \mathrm{H}_{6} \mathrm{~F}_{3} \mathrm{O}\right]^{+}, 89(63)\left[\mathrm{C}_{4} \mathrm{H}_{9} \mathrm{O}_{2}\right]^{+}, 59(100)\left[\mathrm{C}_{3} \mathrm{H}_{7} \mathrm{O}\right]^{+} ; \mathrm{t}_{\mathrm{R}}(\mathrm{GC})=12.49$ minutes.

General Procedure B: Difluorinated Diol Preparation. $\left(1 R^{*}, 3 R^{*}, 4 a S^{*}, 8 a S^{*}\right)-2,2-$ difluoro-3-methyloctahydronaphthalene-1,8a(1H)-diol (1, 3-trans, 1, 8a-trans-4a) and 
$\left(1 R^{*}, 3 S^{*}, 4 a S^{*}, 8 a S^{*}\right)$-2,2-difluoro-3-methyloctahydronaphthalene-1,8a(1H)-diol (1, 3-cis, 1, 8a-trans-4a).1,3-Bis(2,6-diisopropylphenyl-imidazol-2-ylidene)gold(I) chloride $(5 \mathrm{~mol} \%$, $0.031 \mathrm{~g})$ was taken up in dichloromethane $(1 \mathrm{~mL})$ and added via syringe to dichloromethane (3.4 mL). The solution was stirred at room temperature then a solution of silver hexafluoroantimonate(V) $(5 \mathrm{~mol} \%, 0.017 \mathrm{~g})$ in methanol $(0.6 \mathrm{~mL})$ was added as a stream via syringe. An off-white precipitate formed after the addition was complete. The flask was fitted with an air condenser (open to the atmosphere), and the suspension was heated to $40{ }^{\circ} \mathrm{C}$. A solution of allyl alcohol trans-3a and cis-3a (0.306 g, $1 \mathrm{mmol})$ in dichloromethane $(1 \mathrm{~mL})$ was added in a stream via syringe. After stirring for 5 hours at $40{ }^{\circ} \mathrm{C}$ the reaction was allowed to cool to room temperature. Solid tetrabutylammonium borohydride $(0.258 \mathrm{~g}, 1 \mathrm{mmol})$ was added to the flask in small portions. A slight effervescence was observed as the reducing agent was added and the reaction mixture darkened from colourless to dark brown. The reaction mixture was then allowed to stir at room temperature for 18 hours, then was quenched with hydrogen peroxide (10 $\mathrm{mL}$ of a $3 \mathrm{wt} \%$ aqueous solution), followed by sodium hydroxide ( $5 \mathrm{~mL}$ of a $10 \mathrm{wt} \%$ aqueous solution). The mixture was transferred to a separating funnel and the organic layer was removed. The aqueous layer was extracted with dichloromethane $(4 \times 40 \mathrm{~mL})$. The combined organic extracts were then washed with sodium sulfite $(10 \mathrm{~mL}$ of a saturated aqueous solution). The organic layer was separated, dried $\left(\mathrm{MgSO}_{4}\right)$ and concentrated under reduced pressure to afford the crude product as aviscous pale yellow oil $(0.280 \mathrm{~g})$. The crude material was purified by flash column chromatography (40 g silica, $2 \%$ acetone in dichloromethane) to afford an inseparable mixture of 1, 3-trans, 1, 8a-trans-4a and 1,3-cis, 1, 8a-trans-4a as a colourless solid $(0.154 \mathrm{~g}, 70 \%, 2.6: 1) . \mathrm{R}_{\mathrm{f}}=$ 0.47 (5\% acetone in dichloromethane); The following signals were attributed to both the minor 1, 3-cis, 1, 8a-trans-diastereoisomer 4a and major 1, 3-trans, 1, 8a-trans diastereoisomer 4a ${ }^{1} \mathrm{H}$ NMR (400 MHz, $\left.\mathrm{CDCl}_{3}\right): \delta=3.60-3.47(\mathrm{~m}, 1 \mathrm{H}), 2.42-2.17(\mathrm{~m}, 1 \mathrm{H})$, 
1.97-1.27 (envelope, 17H) ppm; The following signals were attributed to the major 1, 3-trans, 1, 8a-trans-diastereoisomer 4a (assigned on the basis of $\delta$ and intensity); ${ }^{1} \mathrm{H}$ NMR (400 MHz, $\left.\mathrm{CDCl}_{3}\right): \delta=2.14(\mathrm{t}, J=2.8 \mathrm{~Hz}, 1 \mathrm{H}), 1.06(\mathrm{~d}, J=6.9 \mathrm{~Hz}, 3 \mathrm{H}) \mathrm{ppm} ;{ }^{13} \mathrm{C}$ NMR $(100 \mathrm{MHz}$, $\left.\mathrm{CDCl}_{3}\right): \delta=124.0\left(\mathrm{t},{ }^{1} J_{\mathrm{C}-\mathrm{F}}=248.2 \mathrm{~Hz}\right), 74.2\left(\mathrm{dd},{ }^{2} J_{\mathrm{C}-\mathrm{F}}=27.7,21.4 \mathrm{~Hz}\right), 72.7\left(\mathrm{~d},{ }^{3} J_{\mathrm{C}-\mathrm{F}}=5.2\right.$ $\mathrm{Hz}), 36.6,33.7,32.8\left(\mathrm{t},{ }^{2} J_{\mathrm{C}-\mathrm{F}}=21.4 \mathrm{~Hz}\right), 32.6\left(\mathrm{~d},{ }^{3} J_{\mathrm{C}-\mathrm{F}}=8.5 \mathrm{~Hz}\right), 26.6,25.1,19.8,11.2(\mathrm{~d}$, $\left.{ }^{3} J_{\mathrm{C}-\mathrm{F}}=5.6 \mathrm{~Hz}\right) \mathrm{ppm} ;{ }^{19} \mathrm{~F} \mathrm{NMR}\left(376 \mathrm{MHz}, \mathrm{CDCl}_{3}\right): \delta=-105.9\left(\mathrm{dq},{ }^{2} J=256.5,{ }^{3} J_{\mathrm{F}-\mathrm{H}}={ }^{4} J_{\mathrm{F}-\mathrm{H}}=\right.$ $4.8 \mathrm{~Hz}, 1 \mathrm{~F}),-116.6\left(\mathrm{ddt},{ }^{2} J=256.5,{ }^{3} J_{\mathrm{F}-\mathrm{H}}=30.3,{ }^{3} J_{\mathrm{F}-\mathrm{H}}={ }^{4} J_{\mathrm{F}-\mathrm{H}}=7.6 \mathrm{~Hz}, 1 \mathrm{~F}\right) \mathrm{ppm}$; The following signals were attributed to the minor 1,3-cis, 1, 8a-trans--diastereoisomer $4 \mathbf{4}$ (assigned on the basis of $\delta$ and intensity); ${ }^{1} \mathrm{H}$ NMR (400 MHz, $\mathrm{CDCl}_{3}$ ): $\delta=2.11$ (t, $J=2.7$ $\mathrm{Hz}, 1 \mathrm{H}) \mathrm{ppm} ;{ }^{13} \mathrm{C} \mathrm{NMR}\left(100 \mathrm{MHz}, \mathrm{CDCl}_{3}\right): \delta=123.4\left(\mathrm{dd},{ }^{1} J_{\mathrm{C}-\mathrm{F}}=253.8,242.5 \mathrm{~Hz}\right), 75.3$ $\left(\mathrm{dd},{ }^{2} J_{\mathrm{C}-\mathrm{F}}=28.9,21.7 \mathrm{~Hz}\right), 72.3\left(\mathrm{~d},{ }^{3} J_{\mathrm{C}-\mathrm{F}}=5.8 \mathrm{~Hz}\right), 35.1\left(\mathrm{dd},{ }^{2} J_{\mathrm{C}-\mathrm{F}}=23.4,21.0 \mathrm{~Hz}\right), 31.1$, $30.8\left(\mathrm{~d},{ }^{3} J_{\mathrm{C}-\mathrm{F}}=7.3 \mathrm{~Hz}\right), 26.8,25.3,19.9,14.2\left(\mathrm{dd},{ }^{3} J_{\mathrm{C}-\mathrm{F}}=9.4,3.7 \mathrm{~Hz}\right) \mathrm{ppm} ;{ }^{19} \mathrm{~F}$ NMR $(376$ $\left.\mathrm{MHz}, \mathrm{CDCl}_{3}\right): \delta=-93.6\left(\mathrm{ddt},{ }^{2} J=261.3,{ }^{3} J_{\mathrm{F}-\mathrm{H}}=17.6,{ }^{3} J_{\mathrm{F}-\mathrm{H}}={ }^{4} J_{\mathrm{F}-\mathrm{H}}=8.0 \mathrm{~Hz}\right),-104.5\left(\mathrm{~d},{ }^{2} J=\right.$ 261.3 Hz) ppm; $\bar{v} /($ neat $)=3608,3450,2919,1446,1069,985 \mathrm{~cm}^{-1}$; HRMS (ASAP): calcd for $\mathrm{C}_{11} \mathrm{H}_{17} \mathrm{~F}_{2} \mathrm{O}_{2}, 219.1197[\mathrm{M}-\mathrm{H}]^{+}$, found: 219.1194; MS (EI): $m / z(\%): 220$ (3) [M] $]^{+}, 182$ (13) $[\mathrm{M}-2 \mathrm{~F}]^{+} ; \mathrm{t}_{\mathrm{R}}(\mathrm{GC})=11.27$ minutes; ${ }^{*}$ elemental analysis calcd $(\%)$ for $\mathrm{C}_{11} \mathrm{H}_{18} \mathrm{~F}_{2} \mathrm{O}_{2}$ : $\mathrm{C}$, 59.98; H, 8.24; found: C, 59.75; H, 8.17. This analysis was obtained for the amorphous solid obtained following chromatography so no melting point was recorded.* the individual diastereoisomers appeared as one peak by GC. Diastereomerically pure 1, 3-trans, 1, 8atrans-4a could be obtained by performing three vapour diffusion recrystallizations (chloroform/pentane) of the mixed solid diol $(0.024 \mathrm{~g}, 11 \%) . \mathrm{m} . \mathrm{p} .=84-86{ }^{\circ} \mathrm{C}$ (recrystallized from chloroform/pentane vapour diffusion as a colourless plate); $\mathrm{R}_{\mathrm{f}}=0.47$ (5\% acetone in dichloromethane); ${ }^{1} \mathrm{H}$ NMR (400 $\left.\mathrm{MHz}, \mathrm{CDCl}_{3}\right): \delta=3.54\left(\mathrm{td}, J_{\mathrm{H}-\mathrm{F}}=6.0, J=3.2 \mathrm{~Hz}, 1 \mathrm{H}\right)$, $2.38-2.16(\mathrm{~m}, 1 \mathrm{H}), 2.14\left(\mathrm{td}, J={ }^{4} J_{\mathrm{H}-\mathrm{F}}=3.0,{ }^{4} J_{\mathrm{H}-\mathrm{F}}=1.0 \mathrm{~Hz}, 1 \mathrm{H}\right), 1.85\left(\mathrm{tdd},{ }^{2} J=J=13.3, J=\right.$ $\left.4.9,{ }^{4} J=1.2 \mathrm{~Hz}, 1 \mathrm{H}\right), 1.80-1.19$ (envelope, $\left.11 \mathrm{H}\right), 1.08(\mathrm{~d}, J=6.9 \mathrm{~Hz}, 3 \mathrm{H}) \mathrm{ppm} ;{ }^{13} \mathrm{C} \mathrm{NMR}$ 
$\left(100 \mathrm{MHz}, \mathrm{CDCl}_{3}\right): \delta=124.0\left(\mathrm{t},{ }^{1} J_{\mathrm{C}-\mathrm{F}}=248.2 \mathrm{~Hz}\right), 74.2\left(\mathrm{dd},{ }^{2} J_{\mathrm{C}-\mathrm{F}}=27.7,21.4 \mathrm{~Hz}\right), 72.7(\mathrm{~d}$, $\left.{ }^{3} J_{\mathrm{C}-\mathrm{F}}=5.2 \mathrm{~Hz}\right), 36.6,33.7,32.8\left(\mathrm{t},{ }^{2} J_{\mathrm{C}-\mathrm{F}}=21.4 \mathrm{~Hz}\right), 32.6\left(\mathrm{~d},{ }^{3} J_{\mathrm{C}-\mathrm{F}}=8.5 \mathrm{~Hz}\right), 26.6,25.1,19.8$, $11.2\left(\mathrm{~d},{ }^{3} J_{\mathrm{C}-\mathrm{F}}=5.6 \mathrm{~Hz}\right)$ ppm; ${ }^{19} \mathrm{~F} \mathrm{NMR}\left(376 \mathrm{MHz}, \mathrm{CDCl}_{3}\right): \delta=-105.9\left(\mathrm{dq},{ }^{2} J=256.5,{ }^{3} J_{\mathrm{F}-\mathrm{H}}\right.$ $\left.={ }^{4} J_{\mathrm{F}-\mathrm{H}}=4.8 \mathrm{~Hz}\right),-116.6\left(\mathrm{ddt},{ }^{2} J=256.5,{ }^{3} J_{\mathrm{F}-\mathrm{H}}=30.3,{ }^{3} J_{\mathrm{F}-\mathrm{H}}={ }^{4} J_{\mathrm{F}-\mathrm{H}}=7.6 \mathrm{~Hz}\right) \mathrm{ppm} ; \bar{v} /($ neat $)=$ $3617,3450,2922,1446,1068,985,957 \mathrm{~cm}^{-1}$; MS (EI): $m / z(\%): 220$ (2) [M] $]^{+}, 182$ (19) [M$2 \mathrm{~F}]^{+} ; \mathrm{t}_{\mathrm{R}}(\mathrm{GC})=11.27$ minutes; elemental analysis calcd $(\%)$ for $\mathrm{C}_{11} \mathrm{H}_{18} \mathrm{~F}_{2} \mathrm{O}_{2}: \mathrm{C}, 59.98 ; \mathrm{H}$, 8.24; found: $\mathrm{C}, 60.12 ; \mathrm{H}, 8.48$.

$\left(2 R^{*}, 4 R^{*}, 4 a S^{*}, 9 a S^{*}\right)-3,3-D i f l u o r o-2-m e t h y l d e c a h y d r o-4 a H-b e n z o[7]$ annulene-4,4a-diol (1, 3-trans, 1, 9a-trans-4b) and $\left(2 S^{*}, 4 R^{*}, 4 a S^{*}, 9 a S^{*}\right)-3,3-d i f l u o r o-2-m e t h y l d e c a h y d r o-4 a H$ benzo[7]annulene-4,4a-diol (1, 3-cis, 1, 9a-trans-4b). Prepared according to general procedure B from trans-3b and cis-3b $(0.320 \mathrm{~g}, 1.00 \mathrm{mmol})$ with 1,3-bis(2,6-diisopropylphenylimidazol-2-ylidene)gold(I) chloride (5 mol \%, $0.031 \mathrm{~g})$ and silver hexafluoroantimonate(V) $(5 \mathrm{~mol} \%, 0.017 \mathrm{~g})$ in dichloromethane $(5.4 \mathrm{~mL})$ and methanol $(0.6 \mathrm{~mL})$. After stirring for 5 hours at $40{ }^{\circ} \mathrm{C}$ the reaction was allowed to cool to room temperature and reduced with tetrabutylammonium borohydride $(0.258 \mathrm{~g}, 1 \mathrm{mmol})$. The usual work up afforded a viscous pale yellow oil $(0.323 \mathrm{~g})$ which was purified by flash column chromatography (40 g silica, 1 $\%$ acetone in dichloromethane) to afford an inseparable mixture of 1, 3-trans, 1, 9a-trans-4b and 1, 3-cis, 1, 9a-trans-4b as a colourless solid $(0.162 \mathrm{~g}, 69 \%, 4.9: 1) . \mathrm{R}_{\mathrm{f}}=0.34(1 \%$ acetone in dichloromethane); The following signals were attributed to both the minor 1, 3-cis, 1, 9atrans-diastereoisomer $\mathbf{4 b}$ and major 1, 3-trans, 1, 9a-trans-diastereoisomer $\mathbf{4 b}{ }^{1} \mathrm{H}$ NMR (400 $\left.\mathrm{MHz}, \mathrm{CDCl}_{3}\right): \delta=3.52\left(\mathrm{td}, J_{\mathrm{H}-\mathrm{F}}=5.9, J=3.2 \mathrm{~Hz}, 1 \mathrm{H}\right), 1.99-1.17$ (envelope, $\left.15 \mathrm{H}\right) \mathrm{ppm}$; The following signals were attributed to the major 1, 3-trans, 1, 9a-trans-diastereoisomer $\mathbf{4 b}$ (assigned on the basis of $\delta$ and intensity); ${ }^{1} \mathrm{H}$ NMR (400 MHz, $\left.\mathrm{CDCl}_{3}\right): \delta=2.35-2.14(\mathrm{~m}$, including $\left.2.29\left(\mathrm{t}, J={ }^{4} J_{\mathrm{H}-\mathrm{F}}=3.2 \mathrm{~Hz}, 1 \mathrm{H}\right), 1 \mathrm{H}\right), 2.09-1.99(\mathrm{~m}, 1 \mathrm{H}), 1.04(\mathrm{~d}, J=6.8 \mathrm{~Hz}, 3 \mathrm{H})$ ppm; ${ }^{13} \mathrm{C}$ NMR (100 MHz, MeOD): $\delta=123.1\left(\mathrm{t},{ }^{1} J_{\mathrm{C}-\mathrm{F}}=248.3 \mathrm{~Hz}\right), 75.5\left(\mathrm{dd},{ }^{2} J_{\mathrm{C}-\mathrm{F}}=27.6\right.$, 
$20.5 \mathrm{~Hz}), 74.6\left(\mathrm{~d},{ }^{3} J_{\mathrm{C}-\mathrm{F}}=5.5 \mathrm{~Hz}\right), 40.2,39.2,34.3\left(\mathrm{~d},{ }^{3} J_{\mathrm{C}-\mathrm{F}}=8.5 \mathrm{~Hz}\right), 32.6\left(\mathrm{t},{ }^{2} J_{\mathrm{C}-\mathrm{F}}=22.0\right.$ $\mathrm{Hz}), 28.0,27.0,26.1,19.8,10.2\left(\mathrm{~d},{ }^{3} J_{\mathrm{C}-\mathrm{F}}=5.7 \mathrm{~Hz}\right) \mathrm{ppm} ;{ }^{19} \mathrm{~F} \mathrm{NMR}\left(376 \mathrm{MHz}, \mathrm{CDCl}_{3}\right): \delta=-$ 107.6 (app. ds, $\left.{ }^{2} J=256.3,{ }^{3} J_{\mathrm{F}-\mathrm{H}}={ }^{4} J_{\mathrm{F}-\mathrm{H}}=3.2 \mathrm{~Hz}, 1 \mathrm{~F}\right),-117.1\left(\mathrm{dddd},{ }^{2} J=256.3,{ }^{3} J_{\mathrm{F}-\mathrm{H}}=30.2\right.$, $\left.{ }^{3} J_{\mathrm{F}-\mathrm{H}}=8.9,{ }^{4} J_{\mathrm{F}-\mathrm{H}}=6.0 \mathrm{~Hz}, 1 \mathrm{~F}\right) \mathrm{ppm}$; The following signals were attributed to the minor 1 , 3cis, 1, 9a-trans-diastereoisomer $\mathbf{4 b}$ (assigned on the basis of $\delta$ and intensity); ${ }^{13} \mathrm{C}$ NMR (100 MHz, MeOD): $\delta=122.7\left(\mathrm{dd},{ }^{1} J_{\mathrm{C}-\mathrm{F}}=251.0,243.2 \mathrm{~Hz}\right), 76.6\left(\mathrm{dd},{ }^{2} J_{\mathrm{C}-\mathrm{F}}=28.7,20.5 \mathrm{~Hz}\right), 74.3$ $\left(\mathrm{d},{ }^{3} J_{\mathrm{C}-\mathrm{F}}=5.4 \mathrm{~Hz}\right), 39.0,35.0,34.9\left(\mathrm{dd},{ }^{2} J_{\mathrm{C}-\mathrm{F}}=23.8,21.1 \mathrm{~Hz}\right), 32.6\left(\mathrm{~d},{ }^{3} J_{\mathrm{C}-\mathrm{F}}=6.4 \mathrm{~Hz}\right), 28.2$, 27.1, 26.3, $13.4\left(\mathrm{dd},{ }^{3} J_{\mathrm{C}-\mathrm{F}}=9.7,4.0 \mathrm{~Hz}\right) \mathrm{ppm} ;{ }^{19} \mathrm{~F} \mathrm{NMR}\left(376 \mathrm{MHz}, \mathrm{CDCl}_{3}\right): \delta=-93.7\left(\mathrm{ddt},{ }^{2} J\right.$ $\left.=261.3,{ }^{3} J_{\mathrm{F}-\mathrm{H}}=18.3,{ }^{3} J_{\mathrm{F}-\mathrm{H}}={ }^{4} J_{\mathrm{F}-\mathrm{H}}=8.1 \mathrm{~Hz}\right),-106.5\left(\mathrm{~d},{ }^{2} J=261.3 \mathrm{~Hz}\right) \mathrm{ppm} ; \bar{v} /($ neat $)=3601$, 3333, 2908, 1463, 1084, $979 \mathrm{~cm}^{-1}$; HRMS (APCI): calcd for $\mathrm{C}_{12} \mathrm{H}_{20} \mathrm{~F}_{2} \mathrm{O}_{2}, 234.1431[\mathrm{M}]^{+}$, found: 234.1432; MS (EI): $m / z(\%): 234$ (1) $[\mathrm{M}]^{+}, 196(9)[\mathrm{M}-2 \mathrm{~F}]^{+} ; \mathrm{t}_{\mathrm{R}}(\mathrm{GC})=12.80$ minutes.* elemental analysis calcd (\%) for $\mathrm{C}_{12} \mathrm{H}_{20} \mathrm{~F}_{2} \mathrm{O}_{2}$ : C, 61.52; $\mathrm{H}, 8.60$; found: $\mathrm{C}, 61.40$; $\mathrm{H}$, 8.38. This analysis was obtained for the amorphous solid obtained following chromatography so no melting point was recorded.* the individual diastereoisomers appeared as one peak by GC. Diastereomerically pure 1, 3-trans, 1, 9a-trans-4b could be obtained by performing three vapour diffusion recrystallizations (chloroform/pentane) of the mixed solid diol $(0.018 \mathrm{~g}, 7 \%)$. m.p. $=96-98{ }^{\circ} \mathrm{C}$ (recrystallized from chloroform/pentane vapour diffusion as a colourless plate); $\mathrm{R}_{\mathrm{f}}=0.34\left(1 \%\right.$ acetone in dichloromethane); ${ }^{1} \mathrm{H}$ NMR (400 MHz, $\left.\mathrm{CDCl}_{3}\right): \delta=3.54\left(\mathrm{td}, J_{\mathrm{H}-\mathrm{F}}=5.9, J=3.2 \mathrm{~Hz}, 1 \mathrm{H}\right), 2.36-2.15(\mathrm{~m}, 2 \mathrm{H}), 2.12-1.99(\mathrm{~m}, 1 \mathrm{H}), 1.90-$ 1.23 (envelope, $13 \mathrm{H}), 1.06(\mathrm{~d}, J=6.8 \mathrm{~Hz}, 3 \mathrm{H}) \mathrm{ppm} ;{ }^{13} \mathrm{C} \mathrm{NMR}\left(100 \mathrm{MHz}, \mathrm{CDCl}_{3}\right): \delta=124.0$ $\left(\mathrm{t},{ }^{1} J_{\mathrm{C}-\mathrm{F}}=247.8 \mathrm{~Hz}\right), 76.1\left(\mathrm{dd},{ }^{2} J_{\mathrm{C}-\mathrm{F}}=27.4,20.8 \mathrm{~Hz}\right), 74.8\left(\mathrm{~d},{ }^{3} J_{\mathrm{C}-\mathrm{F}}=5.1 \mathrm{~Hz}\right), 40.2,39.2,34.3$ $\left(\mathrm{d},{ }^{3} J_{\mathrm{C}-\mathrm{F}}=8.6 \mathrm{~Hz}\right), 32.5\left(\mathrm{t},{ }^{2} J_{\mathrm{C}-\mathrm{F}}=21.7 \mathrm{~Hz}\right), 28.1,27.2,26.3,20.0,11.0\left(\mathrm{~d},{ }^{3} J_{\mathrm{C}-\mathrm{F}}=5.2 \mathrm{~Hz}\right)$ ppm; ${ }^{19} \mathrm{~F}$ NMR $\left(376 \mathrm{MHz}, \mathrm{CDCl}_{3}\right): \delta==-107.6$ (app. ds, ${ }^{2} J=256.3,{ }^{3} J_{\mathrm{F}-\mathrm{H}}={ }^{4} J_{\mathrm{F}-\mathrm{H}}=3.2 \mathrm{~Hz}$, 1F), $-117.1\left(\mathrm{dddd},{ }^{2} J=256.3,{ }^{3} J_{\mathrm{F}-\mathrm{H}}=30.2,{ }^{3} J_{\mathrm{F}-\mathrm{H}}=8.9,{ }^{4} J_{\mathrm{F}-\mathrm{H}}=6.0 \mathrm{~Hz}, 1 \mathrm{~F}\right) \mathrm{ppm} ; \bar{v} /($ neat $)=$ 3601, 3333, 2908, 1463, 1084, $979 \mathrm{~cm}^{-1}$; MS (EI): $m / z(\%): 234(1)[\mathrm{M}]^{+}, 196(9)[\mathrm{M}-2 \mathrm{~F}]^{+} ; \mathrm{t}_{\mathrm{R}}$ 
$(\mathrm{GC})=12.80$ minutes. elemental analysis calcd $(\%)$ for $\mathrm{C}_{12} \mathrm{H}_{20} \mathrm{~F}_{2} \mathrm{O}_{2}: \mathrm{C}, 61.52 ; \mathrm{H}, 8.60$; found: $\mathrm{C}, 61.37 ; \mathrm{H}, 8.50$.

$\left(4 a S^{*}, 5 R^{*}, 7 R^{*}, 8 a R^{*}\right)-6,6-D i f l u o r o-7-m e t h y l o c t a h y d r o-4 a H-i s o c h r o m e n e-4 a, 5-d i o l$

(4a, 5-trans, 5, 7-trans-4c), (4aS*,5R*,7S*,8aR*)-6,6-difluoro-7-methyloctahydro-4aHisochromene-4a,5-diol (4a, 5-trans, 5, 7-cis-4c) and (4aS*,5S*,7R*,8aR*)-6,6-difluoro-7methyloctahydro-4aH-isochromene-4a,5-diol (4a, 5-cis, 5, 7-cis-4c). Prepared according to general procedure B from trans-3c and cis-3c (0.308 g, $1.00 \mathrm{mmol})$ with 1,3-bis(2,6diisopropylphenyl-imidazol-2-ylidene)gold(I) chloride $(5 \mathrm{~mol} \%, 0.031 \mathrm{~g})$ and silver hexafluoroantimonate(V) $(5 \mathrm{~mol} \%, 0.017 \mathrm{~g})$ in dichloromethane $(5.4 \mathrm{~mL})$ and methanol $(0.6$ $\mathrm{mL}$ ). After stirring for 6.5 hours at $40{ }^{\circ} \mathrm{C}$ the reaction was allowed to cool to room temperature and reduced with tetrabutylammonium borohydride $(0.258 \mathrm{~g}, 1 \mathrm{mmol})$. The usual work up afforded a viscous pale yellow oil $(0.300 \mathrm{~g})$ which was purified by flash column chromatography (40 g silica, $10 \%$ acetone in dichloromethane) to afford an inseparable mixture of 4a, 5-trans, 5, 7-trans-4c, 4a, 5-trans, 5, 7-cis-4c and 4a, 5-cis, 5, 7-cis-4c as a colourless solid (0.107 g, $48 \%, 6.7: 1.4: 1) . \mathrm{R}_{\mathrm{f}}=0.39$ (20\% acetone in dichloromethane); The following signals were attributed to the minor 4a, 5-trans, 5, 7-cis -4c and 4a, 5-cis, 5, 7-cis 4c diastereoisomers and the major 4a, 5-trans, 5, 7-trans- diastereoisomer $4 \mathbf{c}{ }^{1} \mathrm{H}$ NMR (400 $\left.\mathrm{MHz}, \mathrm{CDCl}_{3}\right): \delta=3.93-3.78(\mathrm{~m}, 3 \mathrm{H}), 3.65-3.46(\mathrm{~m}, 4 \mathrm{H}), 2.47-1.47$ (envelope, $\left.6 \mathrm{H}\right), 1.43-$ 1.15 (envelope, 4H) ppm; The following signals were attributed to the major 4a, 5-trans, 5, 7trans diastereoisomer $4 \mathbf{c}$ (assigned on the basis of $\delta$ and intensity); ${ }^{1} \mathrm{H}$ NMR (400 MHz, $\left.\mathrm{CDCl}_{3}\right): \delta=2.70\left(\mathrm{t}, J={ }^{4} J_{\mathrm{H}-\mathrm{F}}=3.0 \mathrm{~Hz}, 1 \mathrm{H}\right), 1.09(\mathrm{~d}, J=6.9 \mathrm{~Hz}, 3 \mathrm{H}) \mathrm{ppm} ;{ }^{13} \mathrm{C} \mathrm{NMR}(100$ $\left.\mathrm{MHz}, \mathrm{CDCl}_{3}\right): \delta=123.7\left(\mathrm{dd},{ }^{1} J_{\mathrm{C}-\mathrm{F}}=250.5,246.7 \mathrm{~Hz}\right), 73.2\left(\mathrm{dd},{ }^{2} J_{\mathrm{C}-\mathrm{F}}=27.7,21.6 \mathrm{~Hz}\right), 70.6$ $\left(\mathrm{d},{ }^{3} J_{\mathrm{C}-\mathrm{F}}=5.7 \mathrm{~Hz}\right), 66.1,62.1,36.5,33.6,32.7\left(\mathrm{t},{ }^{2} J_{\mathrm{C}-\mathrm{F}}=22.1 \mathrm{~Hz}\right), 27.1\left(\mathrm{~d},{ }^{3} J_{\mathrm{C}-\mathrm{F}}=8.3 \mathrm{~Hz}\right)$, $11.2\left(\mathrm{dd},{ }^{3} J_{\mathrm{C}-\mathrm{F}}=6.2,2.8 \mathrm{~Hz}\right) \mathrm{ppm} ;{ }^{19} \mathrm{~F}$ NMR $\left(376 \mathrm{MHz}, \mathrm{CDCl}_{3}\right): \delta=-105.8\left(\mathrm{dq},{ }^{2} J=258.4\right.$, $\left.{ }^{3} J_{\mathrm{F}-\mathrm{H}}={ }^{4} J_{\mathrm{F}-\mathrm{H}}=4.2 \mathrm{~Hz}, 1 \mathrm{~F}\right),-117.1\left(\mathrm{dddd},{ }^{2} J=258.4,{ }^{3} J_{\mathrm{F}-\mathrm{H}}=30.5,{ }^{3} J_{\mathrm{F}-\mathrm{H}}=10.7,{ }^{4} J_{\mathrm{F}-\mathrm{H}}=5.9 \mathrm{~Hz}\right.$, 
1F) ppm; The following signals were attributed to the 4a, 5-trans, 5, 7-cis -diastereoisomer 4c (assigned on the basis of $\delta$ and intensity); ${ }^{1} \mathrm{H} \mathrm{NMR}\left(400 \mathrm{MHz}, \mathrm{CDCl}_{3}\right): \delta=2.65\left(\mathrm{t}, J={ }^{4} J_{\mathrm{H}-\mathrm{F}}\right.$ $=2.9 \mathrm{~Hz}, 1 \mathrm{H}), 1.12(\mathrm{~d}, J=6.9 \mathrm{~Hz}, 3 \mathrm{H}) \mathrm{ppm} ;{ }^{13} \mathrm{C} \mathrm{NMR}\left(100 \mathrm{MHz}, \mathrm{CDCl}_{3}\right): \delta=123.1(\mathrm{dd}$, $\left.{ }^{1} J_{\mathrm{C}-\mathrm{F}}=254.8,241.6 \mathrm{~Hz}\right), 74.3\left(\mathrm{dd},{ }^{2} J_{\mathrm{C}-\mathrm{F}}=29.0,21.9 \mathrm{~Hz}\right), 70.4\left(\mathrm{~d},{ }^{3} J_{\mathrm{C}-\mathrm{F}}=7.7 \mathrm{~Hz}\right), 66.5,62.2$, $41.1,34.8\left(\mathrm{t},{ }^{2} J_{\mathrm{C}-\mathrm{F}}=22.5 \mathrm{~Hz}\right), 31.3,27.0\left(\mathrm{~d},{ }^{3} J_{\mathrm{C}-\mathrm{F}}=8.3 \mathrm{~Hz}\right), 14.1\left(\mathrm{dd},{ }^{3} J_{\mathrm{C}-\mathrm{F}}=9.9,4.0 \mathrm{~Hz}\right)$ ppm; ${ }^{19} \mathrm{~F} \mathrm{NMR}\left(376 \mathrm{MHz}, \mathrm{CDCl}_{3}\right): \delta=-93.7\left(\mathrm{dddd},{ }^{2} J=263.9,{ }^{3} J_{\mathrm{F}-\mathrm{H}}=18.0,10.4,{ }^{4} J_{\mathrm{F}-\mathrm{H}}=6.7\right.$ $\mathrm{Hz}, 1 \mathrm{~F}),-104.3\left(\mathrm{~d},{ }^{2} J=263.9 \mathrm{~Hz}, 1 \mathrm{~F}\right) \mathrm{ppm}$; The following signals were attributed to the $4 \mathrm{a}$, 5-cis, 5, 7-cis-diastereoisomer 4c (assigned on the basis of $\delta$ and intensity); ${ }^{1} \mathrm{H}$ NMR (400 $\left.\mathrm{MHz}, \mathrm{CDCl}_{3}\right): \delta=2.84(\mathrm{~d}, J=6.5 \mathrm{~Hz}, 1 \mathrm{H}) \mathrm{ppm} ;{ }^{13} \mathrm{C} \mathrm{NMR}\left(100 \mathrm{MHz}, \mathrm{CDCl}_{3}\right): \delta=75.0(\mathrm{t}$, $\left.{ }^{2} J_{\mathrm{C}-\mathrm{F}}=19.3 \mathrm{~Hz}\right), 70.2\left(\mathrm{~d},{ }^{3} J_{\mathrm{C}-\mathrm{F}}=5.4 \mathrm{~Hz}\right), 65.6,62.7,36.8\left(\mathrm{dd},{ }^{2} J_{\mathrm{C}-\mathrm{F}}=23.3,21.2 \mathrm{~Hz}\right), 34.9$, 29.2, $25.3\left(\mathrm{~d},{ }^{3} J_{\mathrm{C}-\mathrm{F}}=7.0 \mathrm{~Hz}\right), 11.3\left(\mathrm{dd},{ }^{3} J_{\mathrm{C}-\mathrm{F}}=6.2,2.9 \mathrm{~Hz}\right) \mathrm{ppm} ;{ }^{19} \mathrm{~F}$ NMR $(376 \mathrm{MHz}$, $\left.\mathrm{CDCl}_{3}\right): \delta=(-106.7)-(-107.6)\left(\mathrm{m}\right.$, including -107.1 (app. d, $\left.\left.{ }^{2} J=243.7 \mathrm{~Hz}, 1 \mathrm{~F}\right)\right),-104.4$ $\left(\mathrm{dddd},{ }^{2} J=243.7,{ }^{3} J_{\mathrm{F}-\mathrm{H}}=28.2,20.1,{ }^{4} J_{\mathrm{F}-\mathrm{H}}=4.6 \mathrm{~Hz}, 1 \mathrm{~F}\right) \mathrm{ppm} ; \bar{v} /($ neat $)=3331,2878,2908$, 1472, 1091, $966 \mathrm{~cm}^{-1}$; HRMS (APCI): calcd for $\mathrm{C}_{10} \mathrm{H}_{17} \mathrm{~F}_{2} \mathrm{O}_{3}, 223.1146[\mathrm{M}+\mathrm{H}]^{+}$, found: 223.1142; MS (EI): $m / z$ (\%): 222 (1) $[\mathrm{M}]^{+}$(major 4a, 5-trans, 5, 7-trans diastereoisomer, minor 4a, 5-trans, 5, 7-cis diastereoisomer and minor 4a, 5-cis, 5, 7-cis diastereoisomer); $\mathrm{t}_{\mathrm{R}}$ $(\mathrm{GC})=12.27$ minutes (major 4a, 5-trans, 5, 7-trans diastereoisomer), 12.34 minutes (minor 4a, 5-trans, 5, 7-cis diastereoisomer), 12.69 (minor 4a, 5-cis, 5, 7-cis diastereoisomer); elemental analysis calcd (\%) for $\mathrm{C}_{10} \mathrm{H}_{16} \mathrm{~F}_{2} \mathrm{O}_{3}$ : C, 54.05; H, 7.26; found: $\mathrm{C}, 54.05 ; \mathrm{H}, 6.93$. This analysis was obtained for the amorphous solid obtained following chromatography so no melting point was recorded.

tert-Butyl $\left(4 a S^{*}, 5 R^{*}, 7 R^{*}, 8 a R^{*}\right)-6,6$-difluoro-4a,5-dihydroxy-7methyloctahydroisoquinoline-2(1H)-carboxylate (4a, 5-trans, 5, 7-trans-4d), tert-butyl $\left(4 a S^{*}, 5 R^{*}, 7 S^{*}, 8 a R^{*}\right)-6,6$-difluoro-4a,5-dihydroxy-7-methyloctahydroisoquinoline-2(1H)carboxylate (4a, 5-trans, 5, 7-cis-4d) and tert-butyl $\left(4 a S^{*}, 5 S^{*}, 7 R^{*}, 8 a R^{*}\right)$-6,6-difluoro-4a,5- 
dihydroxy-7-methyloctahydroisoquinoline-2(1H)-carboxylate (4a, 5-cis, 5, 7-cis-4d). Prepared according to general procedure B from trans-3d and cis-3d $(0.407 \mathrm{~g}, 1.00 \mathrm{mmol})$ with 1,3-bis(2,6-diisopropylphenyl-imidazol-2-ylidene)gold(I) chloride (5 mol \%, $0.031 \mathrm{~g}$ ) and silver hexafluoroantimonate(V) $(5 \mathrm{~mol} \%, 0.017 \mathrm{~g})$ in dichloromethane $(5.4 \mathrm{~mL})$ and methanol $(0.6 \mathrm{~mL})$. After stirring for 240 hours at $40{ }^{\circ} \mathrm{C}$ the reaction was allowed to cool to room temperature and reduced with tetrabutylammonium borohydride $(0.258 \mathrm{~g}, 1 \mathrm{mmol})$. The usual work up afforded a viscous pale yellow oil $(0.434 \mathrm{~g})$ which was purified by flash column chromatography (40 g silica, 5-8 \% acetone in dichloromethane) to afford an inseparable mixture of 4a, 5-trans, 5, 7-trans-4d, 4a, 5-trans, 5, 7-cis-4d and 4a, 5-cis, 5, 7cis-4d as a colourless solid $(0.160 \mathrm{~g}, 50 \%, 3.4: 0.9: 1) . \mathrm{R}_{\mathrm{f}}=0.31(10 \%$ acetone in dichloromethane); The following signals were attributed to the minor 4a, 5-trans, 5, 7-cis-4d and 4a, 5-cis, 5, 7-cis-4d diastereoisomers and the major 4a, 5-trans, 5, 7-transdiastereoisomer 4d ${ }^{1} \mathrm{H}$ NMR (600 MHz, pyridine- $\left.d_{5}, 373 \mathrm{~K}\right): \delta=4.35-4.21(\mathrm{~m}, 1 \mathrm{H}), 4.20$ $4.05(\mathrm{~m}, 1 \mathrm{H}), 3.69-3.27(\mathrm{~m}, 2 \mathrm{H}), 3.28-3.06(\mathrm{~m}, 1 \mathrm{H}), 2.63-2.35(\mathrm{~m}, 2 \mathrm{H}), 2.33-2.14(\mathrm{~m}, 1 \mathrm{H})$, 2.14-1.77 (m, 1H), 1.77-1.51 (m, including $1.60(\mathrm{~s}, 9 \mathrm{H}), 15 \mathrm{H}), 1.39-1.22(\mathrm{~m}, 2 \mathrm{H}) \mathrm{ppm}$; The following signals were attributed to the major 4a, 5-trans, 5, 7-trans-diastereoisomer $\mathbf{4 d}$ (assigned on the basis of $\delta$ and intensity); ${ }^{1} \mathrm{H}$ NMR (600 MHz, pyridine- $d_{5}, 373 \mathrm{~K}$ ): $\delta=6.97$ (br. s, 1H), 4.59 (br. s, $1 \mathrm{H}), 3.95\left(\mathrm{t}, J={ }^{4} J_{\mathrm{H}-\mathrm{F}}=6.2 \mathrm{~Hz}, 1 \mathrm{H}\right), 1.17(\mathrm{~d}, J=7.0 \mathrm{~Hz}, 3 \mathrm{H}) \mathrm{ppm}$; ${ }^{13} \mathrm{C} \mathrm{NMR}\left(100 \mathrm{MHz}, \mathrm{CDCl}_{3}\right): \delta=154.3,123.8\left(\mathrm{dd},{ }^{1} J_{\mathrm{C}-\mathrm{F}}=251.5,246.5 \mathrm{~Hz}\right), 79.1,73.3(\mathrm{dd}$, $\left.{ }^{2} J_{\mathrm{C}-\mathrm{F}}=27.8,21.9 \mathrm{~Hz}\right), 71.2\left(\mathrm{~d},{ }^{3} J_{\mathrm{C}-\mathrm{F}}=5.7 \mathrm{~Hz}\right), 11.1\left(\mathrm{~d},{ }^{3} J_{\mathrm{C}-\mathrm{F}}=5.1 \mathrm{~Hz}\right) \mathrm{ppm}$; The ${ }^{13} \mathrm{C} \mathrm{NMR}$ spectral region ranging from $46-26 \mathrm{ppm}$ was poorly resolved at $298 \mathrm{~K}$. The ${ }^{13} \mathrm{C}$ NMR spectrum was recorded in pyridine- $d_{5}$ at $373 \mathrm{~K}$ to resolve this region; ${ }^{13} \mathrm{C}$ NMR $(150 \mathrm{MHz}$, pyridine- $\left.d_{5}, 373 \mathrm{~K}\right): \delta=44.2,39.3,37.4,34.8,33.6\left(\mathrm{t},{ }^{2} J_{\mathrm{C}-\mathrm{F}}=22.5 \mathrm{~Hz}\right), 30.0\left(\mathrm{~d},{ }^{3} J_{\mathrm{C}-\mathrm{F}}=8.4\right.$ $\mathrm{Hz}), 28.4 \mathrm{ppm} ;{ }^{19} \mathrm{~F}$ NMR $\left(376 \mathrm{MHz}, \mathrm{CDCl}_{3}\right): \delta=-106.2\left(\mathrm{dq},{ }^{2} J=258.2,{ }^{3} J_{\mathrm{F}-\mathrm{H}}={ }^{4} J_{\mathrm{F}-\mathrm{H}}=4.7\right.$ $\mathrm{Hz}, 1 \mathrm{~F}),-117.1$ (br. ddt, $\left.{ }^{2} J=258.2,{ }^{3} J_{\mathrm{F}-\mathrm{H}}=30.0,{ }^{3} J_{\mathrm{F}-\mathrm{H}}={ }^{4} J_{\mathrm{F}-\mathrm{H}}=6.5 \mathrm{~Hz}, 1 \mathrm{~F}\right) \mathrm{ppm}\left(\right.$ the ${ }^{19} \mathrm{~F}$ 
NMR spectrum was well resolved at $273 \mathrm{~K}$ and did not require heating. ${ }^{1} \mathrm{H}$ and ${ }^{13} \mathrm{C}$ NMR resolved slightly better when heated to $373 \mathrm{~K}$ ); The following signals were attributed to the 4a, 5-trans, 5, 7-cis-diastereoisomer 4d (assigned on the basis of $\delta$ and intensity); ${ }^{1} \mathrm{H}$ NMR $\left(600 \mathrm{MHz}\right.$, pyridine- $\left.d_{5}, 373 \mathrm{~K}\right): \delta=1.41(\mathrm{~d}, J=7.4 \mathrm{~Hz}, 1 \mathrm{H}) \mathrm{ppm} ;{ }^{13} \mathrm{C}$ NMR $(100 \mathrm{MHz}$, $\left.\mathrm{CDCl}_{3}\right): \delta=79.2,74.4\left(\mathrm{dd},{ }^{2} J_{\mathrm{C}-\mathrm{F}}=28.9,21.7 \mathrm{~Hz}\right), 71.1\left(\mathrm{~d},{ }^{3} J_{\mathrm{C}-\mathrm{F}}=7.9 \mathrm{~Hz}\right), 14.2\left(\mathrm{dd},{ }^{3} J_{\mathrm{C}-\mathrm{F}}=\right.$ 9.9, 3.4 Hz) ppm; The ${ }^{13} \mathrm{C}$ NMR spectral region ranging from 46-26 ppm was poorly resolved at $298 \mathrm{~K}$. The ${ }^{13} \mathrm{C}$ NMR spectrum was recorded in pyridine- $d_{5}$ at $373 \mathrm{~K}$ to resolve this region; ${ }^{13} \mathrm{C}$ NMR (150 MHz, pyridine- $\left.d_{5}, 373 \mathrm{~K}\right): \delta=44.7,39.7,37.7\left(\mathrm{t},{ }^{2} J_{\mathrm{C}-\mathrm{F}}=22.1 \mathrm{~Hz}\right), 35.5,29.8$ $\left(\mathrm{d},{ }^{3} J_{\mathrm{C}-\mathrm{F}}=7.8 \mathrm{~Hz}\right) \mathrm{ppm} ;{ }^{19} \mathrm{~F} \mathrm{NMR}\left(376 \mathrm{MHz}, \mathrm{CDCl}_{3}\right): \delta=-93.1\left(\mathrm{dddd},{ }^{2} J=263.9,{ }^{3} J_{\mathrm{F}-\mathrm{H}}=\right.$ $\left.18.0,10.4,{ }^{4} J_{\mathrm{F}-\mathrm{H}}=6.8 \mathrm{~Hz}, 1 \mathrm{~F}\right),-104.7\left(\mathrm{~d},{ }^{2} J=263.9 \mathrm{~Hz}, 1 \mathrm{~F}\right) \mathrm{ppm}$; The following signals were attributed to the 4a, 5-cis, 5, 7-cis-diastereoisomer 4d (assigned on the basis of $\delta$ and intensity); ${ }^{1} \mathrm{H}$ NMR (600 MHz, pyridine- $d 5,373 \mathrm{~K}$ ): $\delta=4.63$ (br. s, $\left.1 \mathrm{H}\right), 3.99\left(\mathrm{t}, J={ }^{4} J_{\mathrm{H}-\mathrm{F}}=\right.$ $6.3 \mathrm{~Hz}, 1 \mathrm{H}), 3.76\left(\mathrm{dd}, J_{\mathrm{H}-\mathrm{F}}=20.2, J=6.3 \mathrm{~Hz}, 1 \mathrm{H}\right), 1.16(\mathrm{~d}, J=6.8 \mathrm{~Hz}, 3 \mathrm{H}) \mathrm{ppm} ;{ }^{13} \mathrm{C} \mathrm{NMR}$ $\left(100 \mathrm{MHz}, \mathrm{CDCl}_{3}\right): \delta=79.1,74.9\left(\mathrm{t},{ }^{2} J_{\mathrm{C}-\mathrm{F}}=20.4 \mathrm{~Hz}\right), 70.8\left(\mathrm{~d},{ }^{3} J_{\mathrm{C}-\mathrm{F}}=5.8 \mathrm{~Hz}\right), 11.3\left(\mathrm{dd},{ }^{3} J_{\mathrm{C}-\mathrm{F}}\right.$ $=5.6,2.9 \mathrm{~Hz}) \mathrm{ppm}$; The ${ }^{13} \mathrm{C}$ NMR spectral region ranging from $46-26 \mathrm{ppm}$ was poorly resolved at $298 \mathrm{~K}$. The ${ }^{13} \mathrm{C}$ NMR spectrum was recorded in pyridine- $d_{5}$ at $373 \mathrm{~K}$ to resolve this region; ${ }^{13} \mathrm{C}$ NMR $(150 \mathrm{MHz}$, pyridine- $d 5,373 \mathrm{~K}): \delta=43.9,41.7,39.3,36.1\left(\mathrm{t},{ }^{2} J_{\mathrm{C}-\mathrm{F}}=\right.$ $23.2 \mathrm{~Hz}), 32.3,28.5\left(\mathrm{~d},{ }^{3} J_{\mathrm{C}-\mathrm{F}}=7.1 \mathrm{~Hz}\right) \mathrm{ppm} ;{ }^{19} \mathrm{~F} \mathrm{NMR}\left(376 \mathrm{MHz}, \mathrm{CDCl}_{3}\right): \delta=-107.6\left(\mathrm{~d},{ }^{2} J=\right.$ $243.7 \mathrm{~Hz}, 1 \mathrm{~F}),-116.1\left(\mathrm{ddd},{ }^{2} J=243.7,{ }^{3} J_{\mathrm{F}-\mathrm{H}}=27.4,20.2 \mathrm{~Hz}, 1 \mathrm{~F}\right) \mathrm{ppm} ; \bar{v} /($ neat $)=3274,2924$, 1656, 1158, $870 \mathrm{~cm}^{-1}$; HRMS (APCI): calcd for $\mathrm{C}_{15} \mathrm{H}_{26} \mathrm{~F}_{2} \mathrm{O}_{4} \mathrm{~N}_{1}, 322.1830[\mathrm{M}+\mathrm{H}]^{+}$, found:

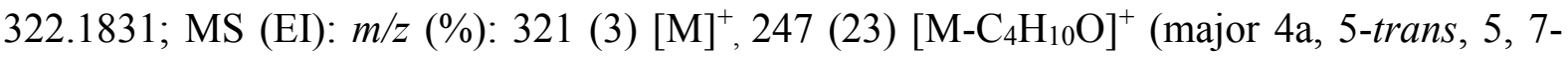
trans diastereoisomer), 321 (1) $[\mathrm{M}]^{+}, 247(24)\left[\mathrm{M}-\mathrm{C}_{4} \mathrm{H}_{10} \mathrm{O}\right]^{+}$(minor 4a, 5-trans, 5, 7-cis diastereoisomer), 321 (2) $[\mathrm{M}]^{+}, 247$ (22) $\left[\mathrm{M}-\mathrm{C}_{4} \mathrm{H}_{10} \mathrm{O}\right]$ (minor 4a, 5-cis, 5, 7-cis diastereoisomer); $\mathfrak{t}_{\mathrm{R}}(\mathrm{GC})=15.06$ minutes (major 4a, 5-trans, 5, 7-trans diastereoisomer), 15.15 minutes (minor 4a, 5-trans, 5, 7-cis diastereoisomer), 14.99 (minor 4a, 5-cis, 5, 7-cis 
diastereoisomer); elemental analysis calcd (\%) for $\mathrm{C}_{15} \mathrm{H}_{25} \mathrm{~F}_{2} \mathrm{NO}_{4}$ : C, 56.06; $\mathrm{H}, 7.84 ; \mathrm{N}, 4.36$; found: C, 56.37; H, 7.86; N, 4.34. This analysis was obtained for the amorphous solid obtained following chromatography so no melting point was recorded.

$\left(1 R^{*}, 2 S^{*}, 4 S^{*}\right)-3,3-D i f l u o r o-1,4-d i m e t h y l c y c l o h e x a n e-1,2-d i o l ~(1,2-t r a n s, 2,4-t r a n s$ 4ea), (1S*,2R*,4S*)-3,3-difluoro-1,4-dimethylcyclohexane-1,2-diol (1, 2-trans, 2, 4-cis-4eb) and $\left(1 R^{*}, 2 R^{*}, 4 S^{*}\right)$-3,3-difluoro-1,4-dimethylcyclohexane-1,2-diol (1, 2-cis, 2, 4-cis-4ec). Prepared according to general procedure B from 3e $(0.327 \mathrm{~g}, 1.23 \mathrm{mmol})$ with 1,3-bis(2,6diisopropylphenyl-imidazol-2-ylidene)gold(I) chloride (5 mol \%, $0.038 \mathrm{~g})$ and silver hexafluoroantimonate(V) $(5 \mathrm{~mol} \%, 0.021 \mathrm{~g})$ in dichloromethane $(6.6 \mathrm{~mL})$ and methanol $(0.7$ $\mathrm{mL}$ ). After stirring for 18 hours at $40{ }^{\circ} \mathrm{C}$ the reaction was allowed to cool to room temperature and reduced with tetrabutylammonium borohydride $(0.317 \mathrm{~g}, 1.23 \mathrm{mmol})$. The usual work up afforded a viscous pale yellow oil $(0.320 \mathrm{~g})$ which was purified by flash column chromatography (40 g silica, $30 \%$ ethyl acetate in hexane) to afford a mixture of 1 , 2-cis, 2, 4-cis-4ec and 1, 2-trans, 2, 4-trans-4ea as a pale yellow oil (33.7 mg, $15 \%$, 1.2:1), 1, 2-trans, 2, 4-trans-4ea as a pale yellow oil (55.5 mg, $25 \%)$ and 1, 2-trans, 2, 4-cis-4eb as a colourless solid (35.6 mg, $16 \%) . \mathrm{R}_{\mathrm{f}}$ 1, 2-trans, 2, 4-trans-4ea $=0.31(10 \%$ acetone in dichloromethane); $\mathrm{R}_{\mathrm{f}}$ 1, 2-cis, 2, 4-cis-4ec $=0.25$ (10\% acetone in dichloromethane); The following signals were attributed to both the major 1, 2-cis, 2, 4-cis-diastereoisomer 4ec and minor 1, 2-trans, 2, 4-trans-diastereoisomer 4ea ${ }^{1} \mathrm{H}$ NMR (400 MHz, $\left.\mathrm{CDCl}_{3}\right): \delta=2.35-1.97$ $(\mathrm{m}, 3 \mathrm{H}), 1.95-1.68(\mathrm{~m}, 3 \mathrm{H}), 1.68-1.42(\mathrm{~m}, 5 \mathrm{H}) \mathrm{ppm}$; The following signals were attributed to the major 1, 2-cis, 2, 4-cis-diastereoisomer 4ec (assigned on the basis of $\delta$ and intensity); ${ }^{1} \mathrm{H}$ $\operatorname{NMR}\left(400 \mathrm{MHz}, \mathrm{CDCl}_{3}\right): \delta=3.48\left(\mathrm{dd}, J_{\mathrm{H}-\mathrm{F}}=20.3,6.0 \mathrm{~Hz}, 1 \mathrm{H}\right), 1.34(\mathrm{~s}, 3 \mathrm{H}), 1.11(\mathrm{~d}, J=6.7$ $\mathrm{Hz}, 3 \mathrm{H}) \mathrm{ppm} ;{ }^{13} \mathrm{C}$ NMR $\left(150 \mathrm{MHz}, \mathrm{CDCl}_{3}\right): \delta=121.9\left(\mathrm{t},{ }^{1} J_{\mathrm{C}-\mathrm{F}}=246.8 \mathrm{~Hz}\right), 74.3\left(\mathrm{t},{ }^{2} J_{\mathrm{C}-\mathrm{F}}=\right.$ $20.3 \mathrm{~Hz}), 71.9\left(\mathrm{~d},{ }^{3} J_{\mathrm{C}-\mathrm{F}}=6.9 \mathrm{~Hz}\right), 36.7\left(\mathrm{dd},{ }^{2} J_{\mathrm{C}-\mathrm{F}}=23.3,20.5 \mathrm{~Hz}\right), 35.3,25.9,24.8\left(\mathrm{~d},{ }^{4} J_{\mathrm{C}-\mathrm{F}}=\right.$ $7.8 \mathrm{~Hz}), 11.0\left(\mathrm{~d},{ }^{3} J_{\mathrm{C}-\mathrm{F}}=4.3 \mathrm{~Hz}\right) \mathrm{ppm} ;{ }^{19} \mathrm{~F}$ NMR $\left(376 \mathrm{MHz}, \mathrm{DMSO}-d_{6}, 373 \mathrm{~K}\right): \delta=-104.6(\mathrm{~d}$, 
$\left.{ }^{2} J=238.7 \mathrm{~Hz}, 1 \mathrm{~F}\right),-127.9\left(\mathrm{dt},{ }^{2} J=238.7,{ }^{3} J_{\mathrm{F}-\mathrm{H}}=20.3 \mathrm{~Hz}, 1 \mathrm{~F}\right) \mathrm{ppm}\left(\right.$ the ${ }^{1} \mathrm{H}$ and ${ }^{13} \mathrm{C} \mathrm{NMR}$ gave clear well resolved spectra at RT and did not require heating. ${ }^{19} \mathrm{~F}$ NMR resolved better when heated to $373 \mathrm{~K}$ ); The following signals were attributed to the minor 1, 2-trans, 2, 4trans-diastereoisomer 4ea (assigned on the basis of $\delta$ and intensity); ${ }^{1} \mathrm{H}$ NMR (400 MHz, $\left.\mathrm{CDCl}_{3}\right): \delta=3.63\left(\mathrm{dd}, J_{\mathrm{H}-\mathrm{F}}=8.1,6.7 \mathrm{~Hz}, 1 \mathrm{H}\right), 1.33(\mathrm{~s}, 3 \mathrm{H}), 1.08(\mathrm{~d}, J=6.9 \mathrm{~Hz}, 3 \mathrm{H}) \mathrm{ppm} ;{ }^{13} \mathrm{C}$ $\operatorname{NMR}\left(150 \mathrm{MHz}, \mathrm{CDCl}_{3}\right): \delta=123.4\left(\mathrm{t},{ }^{1} J_{\mathrm{C}-\mathrm{F}}=248.1 \mathrm{~Hz}\right), 73.4\left(\mathrm{dd},{ }^{2} J_{\mathrm{C}-\mathrm{F}}=26.8,21.6 \mathrm{~Hz}\right)$, $72.3\left(\mathrm{~d},{ }^{3} J_{\mathrm{C}-\mathrm{F}}=4.3 \mathrm{~Hz}\right), 31.9\left(\mathrm{t},{ }^{2} J_{\mathrm{C}-\mathrm{F}}=21.4 \mathrm{~Hz}\right), 31.5,24.7\left(\mathrm{~d},{ }^{3} J_{\mathrm{C}-\mathrm{F}}=7.4 \mathrm{~Hz}\right), 24.7\left(\mathrm{~d},{ }^{4} J_{\mathrm{C}-\mathrm{F}}\right.$ $=7.4 \mathrm{~Hz}), 10.9\left(\mathrm{~d},{ }^{3} J_{\mathrm{C}-\mathrm{F}}=4.5 \mathrm{~Hz}\right) \mathrm{ppm} ;{ }^{19} \mathrm{~F}$ NMR $\left(376 \mathrm{MHz}, \mathrm{DMSO}-d_{6}, 373 \mathrm{~K}\right): \delta=-104.2$ $\left(\mathrm{d},{ }^{2} J=243.2 \mathrm{~Hz}, 1 \mathrm{~F}\right),-114.0\left(\mathrm{dd},{ }^{2} J=243.2,{ }^{3} J_{\mathrm{F}-\mathrm{H}}=25.8 \mathrm{~Hz}, 1 \mathrm{~F}\right) \mathrm{ppm} ; \bar{v} /($ neat $)=3392$, 2941, 1461, 1066, $994 \mathrm{~cm}^{-1}$; HRMS (ESI): calcd for $\mathrm{C}_{8} \mathrm{H}_{18} \mathrm{~F}_{2} \mathrm{O}_{2} \mathrm{~N}_{1}, 198.1300\left[\mathrm{M}+\mathrm{NH}_{4}\right]^{+}$, found: 198.1300; $\mathrm{MS}$ (EI): $m / z \quad(\%): 142$ (8) $[\mathrm{M}-2 \mathrm{~F}]^{+}$(major 1, 2-cis, 2, 4-cis diastereoisomer), $142(7)[\mathrm{M}-2 \mathrm{~F}]^{+}$(minor 1, 2-trans, 2, 4-trans diastereoisomer); $\mathrm{t}_{\mathrm{R}}(\mathrm{GC})=$ 8.77 minutes (major 1, 2-cis, 2, 4-cis diastereoisomer), 8.54 minutes (minor 1, 2-trans, 2, 4trans diastereoisomer). Diastereomerically pure 1, 2-trans, 2, 4-trans-4ea: $\mathrm{R}_{\mathrm{f}}=0.31(10 \%$ acetone in dichloromethane); ${ }^{1} \mathrm{H}$ NMR $\left(400 \mathrm{MHz}, \mathrm{CDCl}_{3}\right): \delta=3.62\left(\mathrm{dd}, J_{\mathrm{H}-\mathrm{F}}=8.1,6.7 \mathrm{~Hz}\right.$, 1H), 2.81 (br. s, 1H), 2.40-1.97 (m, 2H), 1.81-1.69 (m, 1H), 1.67-1.45 (m, 3H), 1.33 (s, 3H), $1.07(\mathrm{~d}, J=6.9 \mathrm{~Hz}, 3 \mathrm{H}) \mathrm{ppm} ;{ }^{13} \mathrm{C} \mathrm{NMR}\left(100 \mathrm{MHz}, \mathrm{CDCl}_{3}\right): \delta=123.9\left(\mathrm{t},{ }^{1} J_{\mathrm{C}-\mathrm{F}}=247.0 \mathrm{~Hz}\right)$, $73.9\left(\mathrm{dd},{ }^{2} J_{\mathrm{C}-\mathrm{F}}=26.9,21.7 \mathrm{~Hz}\right), 72.8\left(\mathrm{~d},{ }^{3} J_{\mathrm{C}-\mathrm{F}}=4.2 \mathrm{~Hz}\right), 32.4\left(\mathrm{t},{ }^{2} J_{\mathrm{C}-\mathrm{F}}=21.8 \mathrm{~Hz}\right), 32.0,25.2$ $\left(\mathrm{d},{ }^{3} J_{\mathrm{C}-\mathrm{F}}=7.3 \mathrm{~Hz}\right), 25.2\left(\mathrm{~d},{ }^{4} J_{\mathrm{C}-\mathrm{F}}=7.3 \mathrm{~Hz}\right), 11.5\left(\mathrm{t},{ }^{3} J_{\mathrm{C}-\mathrm{F}}=4.1 \mathrm{~Hz}\right) \mathrm{ppm} ;{ }^{19} \mathrm{~F} \mathrm{NMR}(376 \mathrm{MHz}$, DMSO- $\left.d_{6}, 373 \mathrm{~K}\right): \delta=-104.2\left(\mathrm{~d},{ }^{2} J=243.2 \mathrm{~Hz}, 1 \mathrm{~F}\right),-114.0\left(\mathrm{dd},{ }^{2} J=243.2,{ }^{3} J_{\mathrm{F}-\mathrm{H}}=25.8 \mathrm{~Hz}\right.$, 1F) $\mathrm{ppm} ; \overline{\mathrm{v}} /($ neat $)=3392,2941,1461,1066,994 \mathrm{~cm}^{-1} ; \mathrm{MS}(\mathrm{EI}): \mathrm{m} / z(\%): 142(7)[\mathrm{M}-2 \mathrm{~F}]^{+}$; $\mathrm{t}_{\mathrm{R}}(\mathrm{GC})=8.54$ minutes; Diastereomerically pure 1, 2-trans, 2, 4-cis-4eb: m.p. $=58-60{ }^{\circ} \mathrm{C}$ (recrystallized from chloroform/pentane vapour diffusion as a colourless plate); $\mathrm{R}_{\mathrm{f}}=0.33$ (20 $\%$ acetone in dichloromethane); ${ }^{1} \mathrm{H}$ NMR $\left(400 \mathrm{MHz}, \mathrm{CDCl}_{3}\right): \delta=3.71\left(\mathrm{dd}, J_{\mathrm{H}-\mathrm{F}}=22.5,4.6\right.$ Hz, 1H), 2.81 (br. s, 1H), 2.64 (s, 1H), 1.99-1.76 (m, 2H), 1.74-1.54 (m, 2H), 1.34 (td, ${ }^{2} J=J$ 
$=13.7, J=4.9 \mathrm{~Hz}, 1 \mathrm{H}) 1.27\left(\mathrm{~d},{ }^{5} J_{\mathrm{H}-\mathrm{F}}=2.3 \mathrm{~Hz}, 3 \mathrm{H}\right), 1.09(\mathrm{~d}, J=6.9 \mathrm{~Hz}, 3 \mathrm{H}) \mathrm{ppm} ;{ }^{13} \mathrm{C} \mathrm{NMR}$ $\left(100 \mathrm{MHz}, \mathrm{CDCl}_{3}\right): \delta=121.9\left(\mathrm{dd},{ }^{1} J_{\mathrm{C}-\mathrm{F}}=249.0,245.2 \mathrm{~Hz}\right), 77.9\left(\mathrm{t},{ }^{2} J_{\mathrm{C}-\mathrm{F}}=20.5 \mathrm{~Hz}\right), 73.4(\mathrm{~d}$, $\left.{ }^{3} J_{\mathrm{C}-\mathrm{F}}=7.8 \mathrm{~Hz}\right), 37.1\left(\mathrm{t},{ }^{2} J_{\mathrm{C}-\mathrm{F}}=21.8 \mathrm{~Hz}\right), 36.2,26.5\left(\mathrm{~d},{ }^{3} J_{\mathrm{C}-\mathrm{F}}=8.5 \mathrm{~Hz}\right), 19.7\left(\mathrm{~d},{ }^{4} J_{\mathrm{C}-\mathrm{F}}=6.7\right.$ $\mathrm{Hz}), 11.3\left(\mathrm{dd},{ }^{3} J_{\mathrm{C}-\mathrm{F}}=5.5,2.4 \mathrm{~Hz}\right) \mathrm{ppm} ;{ }^{19} \mathrm{~F} \mathrm{NMR}\left(376 \mathrm{MHz}, \mathrm{CDCl}_{3}\right): \delta=-108.6\left(\mathrm{~d},{ }^{2} J=\right.$ $239.9 \mathrm{~Hz}, 1 \mathrm{~F}),-131.5\left(\mathrm{dt},{ }^{2} J=239.9,{ }^{3} J_{\mathrm{F}-\mathrm{H}}=22.5 \mathrm{~Hz}, 1 \mathrm{~F}\right) \mathrm{ppm} ; \bar{v} /($ neat $)=3382,2945,1455$, 1031, $988 \mathrm{~cm}^{-1}$; HRMS (ESI): calcd for $\mathrm{C}_{8} \mathrm{H}_{18} \mathrm{~F}_{2} \mathrm{O}_{2} \mathrm{~N}_{1}, 198.1300\left[\mathrm{M}+\mathrm{NH}_{4}\right]^{+}$, found: 198.1300; MS (EI): $m / z(\%): 142(7)[\mathrm{M}-2 \mathrm{~F}]^{+} ; \mathrm{t}_{\mathrm{R}}(\mathrm{GC})=8.96$ minutes.

$\left(1 S^{*}, 2 S^{*}, 4 S^{*}\right)-3,3-$ difluoro-4-methylcyclohexane-1,2-diol (1, 2-cis, 2, 4-trans-4f). Prepared according to general procedure B from $3 \mathbf{f}(0.252 \mathrm{~g}, 1.00 \mathrm{mmol})$ with 1,3-bis(2,6diisopropylphenyl-imidazol-2-ylidene)gold(I) chloride $(5 \mathrm{~mol} \%, 0.031 \mathrm{~g})$ and silver hexafluoroantimonate(V) $(5 \mathrm{~mol} \%, 0.017 \mathrm{~g})$ in dichloromethane $(5.4 \mathrm{~mL})$ and methanol $(0.6$ $\mathrm{mL}$ ). After stirring for 21 hours at $40{ }^{\circ} \mathrm{C}$ the reaction was allowed to cool to room temperature and reduced with tetrabutylammonium borohydride $(0.258 \mathrm{~g}, 1.00 \mathrm{mmol})$. The usual work up afforded a viscous pale yellow oil (0.294 g) which was purified by flash column chromatography (40 g silica, 15-25\% acetone in dichloromethane) to afford 1, 2-cis, 2, 4-trans-4f as a colourless solid $(0.039 \mathrm{~g})$. This material was purified further by recrystallization by vapour diffusion (methanol/pentane) to afford 1, 2-cis, 2, 4-trans-4f as a colourless plate $(0.021 \mathrm{~g}, 11 \%)$. m.p. $=118-120{ }^{\circ} \mathrm{C} ; \mathrm{R}_{\mathrm{f}}=0.42(20 \%$ acetone in dichloromethane); ${ }^{1} \mathrm{H}$ NMR (600 MHz, MeOD): $\delta=3.84\left(\mathrm{dt}, J=8.3, J_{\mathrm{H}-\mathrm{F}}=3.7 \mathrm{~Hz}, 1 \mathrm{H}\right)$, $3.71-3.61(\mathrm{~m}, 1 \mathrm{H}), 2.22-2.06(\mathrm{~m}, 1 \mathrm{H}), 1.77-1.68(\mathrm{~m}, 1 \mathrm{H}), 1.68-1.59(\mathrm{~m}, 2 \mathrm{H}), 1.18\left(\mathrm{qd},{ }^{2} J=J\right.$ $=13.5, J=3.9 \mathrm{~Hz}, 1 \mathrm{H}), 1.01(\mathrm{~d}, J=6.9 \mathrm{~Hz}, 3 \mathrm{H}) \mathrm{ppm} ;{ }^{13} \mathrm{C} \mathrm{NMR}(150 \mathrm{MHz}, \mathrm{MeOD}): \delta=$ $123.5\left(\mathrm{dd},{ }^{1} J_{\mathrm{C}-\mathrm{F}}=252.8,242.5 \mathrm{~Hz}\right), 71.5\left(\mathrm{dd},{ }^{2} J_{\mathrm{C}-\mathrm{F}}=33.8,21.6 \mathrm{~Hz}\right), 69.2\left(\mathrm{~d},{ }^{3} J_{\mathrm{C}-\mathrm{F}}=7.6 \mathrm{~Hz}\right)$, $31.8\left(\mathrm{t},{ }^{2} J_{\mathrm{C}-\mathrm{F}}=22.0 \mathrm{~Hz}\right), 26.7\left(\mathrm{~d},{ }^{3} J_{\mathrm{C}-\mathrm{F}}=8.4 \mathrm{~Hz}\right), 26.5,10.4\left(\mathrm{t},{ }^{3} J_{\mathrm{C}-\mathrm{F}}=3.9 \mathrm{~Hz}\right) \mathrm{ppm} ;{ }^{19} \mathrm{~F}$ NMR (376 MHz, MeOD): $\delta=(-108.9)-(-109.9)\left(\mathrm{m}\right.$, including -109.3 (app. d, $\left.{ }^{2} J=250.3 \mathrm{~Hz}, 1 \mathrm{~F}\right)$ ), $-124.5\left(\mathrm{dd},{ }^{2} J=250.3,{ }^{3} J_{\mathrm{F}-\mathrm{H}}=29.6 \mathrm{~Hz}, 1 \mathrm{~F}\right) \mathrm{ppm} ; \bar{v} /($ neat $)=3400($ broad $), 2963,1457,1046$, 
$957 \mathrm{~cm}^{-1}$; HRMS (APCI): calcd for $\mathrm{C}_{7} \mathrm{H}_{16} \mathrm{~F}_{2} \mathrm{O}_{2} \mathrm{~N}, 184.1144\left[\mathrm{M}+\mathrm{NH}_{4}\right]^{+}$, found: 184.1141; MS (CI): $m / z(\%): 184(100)\left[\mathrm{M}+\mathrm{NH}_{4}\right]^{+} ; \mathrm{t}_{\mathrm{R}}(\mathrm{GC})=9.44$ minutes.

$\left(1 S^{*}, 2 S^{*}, 4 S^{*}\right)$-3,3-difluoro-4,6,6-trimethylcyclohexane-1,2-diol (1, 2-cis, 2, 4-trans4g). Prepared according to general procedure B from $3 \mathrm{~g}(0.280 \mathrm{~g}, 1.00 \mathrm{mmol})$ with 1,3bis(2,6-diisopropylphenyl-imidazol-2-ylidene)gold(I) chloride (5 mol \%, $0.031 \mathrm{~g}$ ) and silver hexafluoroantimonate $(\mathrm{V})(5 \mathrm{~mol} \%, 0.017 \mathrm{~g})$ in dichloromethane $(5.4 \mathrm{~mL})$ and methanol $(0.6$ $\mathrm{mL}$ ). After stirring for 21 hours at $40{ }^{\circ} \mathrm{C}$ the reaction was allowed to cool to room temperature and reduced with tetrabutylammonium borohydride $(0.258 \mathrm{~g}, 1.00 \mathrm{mmol})$. The usual work up afforded a viscous pale yellow oil (0.586 g) which was purified by flash column chromatography (40 g silica, $5 \%$ acetone in dichloromethane) to afford 1, 2-cis, 2, 4trans-4g as a colourless solid $(0.123 \mathrm{~g}, 63 \%) . \mathrm{R}_{\mathrm{f}}=0.25$ (5\% acetone in dichloromethane); ${ }^{1} \mathrm{H}$ NMR $\left(400 \mathrm{MHz}, \mathrm{CDCl}_{3}\right): \delta=4.04\left(\mathrm{tt}, J_{\mathrm{H}-\mathrm{F}}=6.6, J=3.5 \mathrm{~Hz}, 1 \mathrm{H}\right), 3.49-3.38(\mathrm{~m}, 1 \mathrm{H})$, 2.46-2.24 (m, (including 2.39 (app. d, $J=3.5 \mathrm{~Hz}, 1 \mathrm{H})$ and 2.29 (d, $J=9.6 \mathrm{~Hz}, 1 \mathrm{H})$ ), $1 \mathrm{H}), 1.43$ $\left(\mathrm{ddd},{ }^{2} J=14.1,{ }^{4} J_{\mathrm{H}-\mathrm{F}}=6.2, J=4.2 \mathrm{~Hz}, 1 \mathrm{H}\right), 1.26$ (br. t, $\left.{ }^{2} J=J=14.1 \mathrm{~Hz}, 1 \mathrm{H}\right), 1.07(\mathrm{~s}, 3 \mathrm{H})$, $1.06(\mathrm{~d}, J=6.2 \mathrm{~Hz}, 3 \mathrm{H}), 1.03(\mathrm{~s}, 3 \mathrm{H}) \mathrm{ppm} ;{ }^{13} \mathrm{C} \mathrm{NMR}\left(150 \mathrm{MHz}, \mathrm{CDCl}_{3}\right): \delta=122.8\left(\mathrm{dd},{ }^{1} J_{\mathrm{C}-\mathrm{F}}\right.$ $=251.1,243.3 \mathrm{~Hz}), 74.2\left(\mathrm{~d},{ }^{3} J_{\mathrm{C}-\mathrm{F}}=6.7 \mathrm{~Hz}\right), 71.5\left(\mathrm{dd},{ }^{2} J_{\mathrm{C}-\mathrm{F}}=36.3,22.2 \mathrm{~Hz}\right), 41.6\left(\mathrm{~d},{ }^{3} J_{\mathrm{C}-\mathrm{F}}=\right.$ $8.8 \mathrm{~Hz}), 34.8,29.2,28.8\left(\mathrm{t},{ }^{2} J_{\mathrm{C}-\mathrm{F}}=21.5 \mathrm{~Hz}\right), 19.9,10.9\left(\mathrm{t},{ }^{3} J_{\mathrm{C}-\mathrm{F}}=3.7 \mathrm{~Hz}\right) \mathrm{ppm} ;{ }^{19} \mathrm{~F}$ NMR $\left(376 \mathrm{MHz}, \mathrm{CDCl}_{3}\right): \delta=(-109.0)-(-109.9)\left(\mathrm{m}\right.$, including $\left.-109.4\left(\operatorname{app} . \mathrm{d},{ }^{2} J=253.0 \mathrm{~Hz}, 1 \mathrm{~F}\right)\right)$, $-124.5\left(\mathrm{dddd},{ }^{2} J=253.0,{ }^{3} J_{\mathrm{F}-\mathrm{H}}=28.9,6.2,{ }^{4} J_{\mathrm{F}-\mathrm{H}}=4.2 \mathrm{~Hz}, 1 \mathrm{~F}\right) \mathrm{ppm} ; \bar{v} /($ neat $)=3350,2926$, 1465,1371, $1005 \mathrm{~cm}^{-1}$; HRMS (NSI-ES): calcd for $\mathrm{C}_{9} \mathrm{H}_{16} \mathrm{~F}_{2} \mathrm{O}_{2} \mathrm{Na}, 217.1011[\mathrm{M}+\mathrm{Na}]^{+}$, found: 217.1012; $\mathrm{MS}(\mathrm{EI}): \mathrm{m} / z$ (\%): 161(3) $\left[\mathrm{M}-\mathrm{CH}_{3} \mathrm{~F}\right]^{+}, 102$ (61) $\left[\mathrm{M}-\mathrm{C}_{4} \mathrm{H}_{6} \mathrm{~F}_{2}\right]^{+}, 72(100)$ [M$\left.\mathrm{C}_{6} \mathrm{H}_{12} \mathrm{~F}_{2}\right]^{+} ; \mathrm{t}_{\mathrm{R}}(\mathrm{GC})=9.64$ minutes; elemental analysis calcd $(\%)$ for $\mathrm{C}_{9} \mathrm{H}_{16} \mathrm{~F}_{2} \mathrm{O}_{2}: \mathrm{C}, 55.66 ; \mathrm{H}$, 8.30; found: $\mathrm{C}, 55.35 ; \mathrm{H}, 8.23$.

$\left(4 S^{*}, 5 R^{*}, 7 S^{*}\right)-6,6-D i f l u o r o-4,7-d i m e t h y l s p i r o[2.5]$ octane-4,5-diol (4, 5-trans, 5, 7cis-4h), (4S*,5R*,7R*)-6,6-difluoro-4,7-dimethylspiro[2.5]octane-4,5-diol (4, 5-trans, 5, 7- 
trans-4h) and (4R*,5R*,7 *)-6,6-difluoro-4,7-dimethylspiro[2.5]octane-4,5-diol (4, 5-cis, 5, 7-cis-4h). Prepared according to general procedure B from $3 \mathbf{h}(0.352 \mathrm{~g}, 1.21 \mathrm{mmol})$ with 1,3bis(2,6-diisopropylphenyl-imidazol-2-ylidene)gold(I) chloride (5 mol \%, $0.037 \mathrm{~g}$ ) and silver hexafluoroantimonate $(\mathrm{V})(5 \mathrm{~mol} \%, 0.021 \mathrm{~g})$ in dichloromethane $(6.5 \mathrm{~mL})$ and methanol $(0.7$ $\mathrm{mL}$ ). After stirring for 18 hours at $40{ }^{\circ} \mathrm{C}$ the reaction was allowed to cool to room temperature and reduced with tetrabutylammonium borohydride $(0.311 \mathrm{~g}, 1.21 \mathrm{mmol})$. The usual work up afforded a viscous pale yellow oil $(0.564 \mathrm{~g})$ which was purified by flash column chromatography (40 g silica, $6 \%$ acetone in dichloromethane) to afford a mixture of 4, 5-trans, 5, 7-cis-4h, 4, 5-trans, 5, 7-trans-4h and 4, 5-cis, 5, 7-cis-4h (0.125 g). The material was further purified by flash column chromatography (40 g silica, $38 \%$ ethyl acetate in hexane) to afford a mixture of 4, 5-trans, 5, 7-cis-4h, 4, 5-trans, 5, 7-trans-4h and 4, 5-cis, 5, 7-cis-4h (73.4 mg, $29 \%, 1.3: 1: 0.1)$ as a pale yellow oil, 4, 5-trans, 5, 7-cis -4h as a pale yellow oil (13.8 mg, $6 \%)$ and 4, 5-trans, 5, 7-trans-4h as a colourless solid (12.8 mg, $5 \%$ ). $\mathrm{R}_{\mathrm{f}}$ 4, 5-trans, 5, 7-cis-4h $=0.52$ (10\% acetone in dichloromethane); $\mathrm{R}_{\mathrm{f}}$ 4, 5-trans, 5, 7-trans$\mathbf{4 h}=0.61(10 \%$ acetone in dichloromethane $) ; 4,5-c i s, 5,7-c i s-4 \mathbf{h}$ co eluted with the 4, 5trans, 5, 7-trans diastereoisomer; The following signals were attributed to the major 4, 5trans, 5, 7-cis-diastereoisomer $\mathbf{4 h}$ and minor 4, 5-trans, 5, 7-trans-4h and 4, 5-cis, 5, 7-cisdiastereoisomers 4h ${ }^{1} \mathrm{H}$ NMR (600 MHz, DMSO- $\left.d_{6}, 373 \mathrm{~K}\right): \delta=3.63-3.48(\mathrm{~m}, 1 \mathrm{H}), \quad 0.84-$ $0.74(\mathrm{~m}, 2 \mathrm{H}), 0.22-0.06(\mathrm{~m}, 3 \mathrm{H}) \mathrm{ppm}$;

The following signals were attributed to the major 4, 5-trans, 5, 7-cis-diastereoisomer $\mathbf{4 h}$ (assigned on the basis of $\delta$ and intensity); ${ }^{1} \mathrm{H}$ NMR (600 MHz, DMSO- $d_{6}, 373 \mathrm{~K}$ ): $\delta=4.81$ (br. s, 1H), 3.78 (br. s, $1 \mathrm{H}), 2.09-1.91(\mathrm{~m}, 1 \mathrm{H}), 1.75$ (br. t, $\left.{ }^{2} J=J=13.4 \mathrm{~Hz}, 1 \mathrm{H}\right), 1.16\left(\mathrm{~d},{ }^{5} J_{\mathrm{H}-}\right.$ $\mathrm{F}=2.2 \mathrm{~Hz}, 3 \mathrm{H}), 0.97(\mathrm{~d}, J=6.8 \mathrm{~Hz}, 3 \mathrm{H}), 0.91-0.84(\mathrm{~m}, 1 \mathrm{H}), 0.59\left(\mathrm{ddd},{ }^{2} J=9.3, J=5.7,3.8\right.$ $\mathrm{Hz}, 1 \mathrm{H}) \mathrm{ppm} ;{ }^{13} \mathrm{C} \mathrm{NMR}\left(150 \mathrm{MHz}, \mathrm{DMSO}-d_{6}, 373 \mathrm{~K}\right): \delta=123.8\left(\mathrm{t},{ }^{1} J_{\mathrm{C}-\mathrm{F}}=247.0 \mathrm{~Hz}\right), 77.5$ $\left(\mathrm{t},{ }^{2} J_{\mathrm{C}-\mathrm{F}}=19.1 \mathrm{~Hz}\right), 72.2\left(\mathrm{~d},{ }^{3} J_{\mathrm{C}-\mathrm{F}}=8.3 \mathrm{~Hz}\right), 37.8\left(\mathrm{~d},{ }^{3} J_{\mathrm{C}-\mathrm{F}}=8.7 \mathrm{~Hz}\right), 36.7\left(\mathrm{t},{ }^{2} J_{\mathrm{C}-\mathrm{F}}=22.3 \mathrm{~Hz}\right)$, 
25.6, $20.0\left(\mathrm{~d},{ }^{4} J_{\mathrm{C}-\mathrm{F}}=5.6 \mathrm{~Hz}\right), 11.8,8.5,6.6 \mathrm{ppm} ;{ }^{19} \mathrm{~F}$ NMR $\left(376 \mathrm{MHz}, \mathrm{DMSO}-d_{6}, 373 \mathrm{~K}\right): \delta=$ $-104.7\left(\mathrm{dq},{ }^{2} J=240.6,{ }^{3} J_{\mathrm{F}-\mathrm{H}}={ }^{4} J_{\mathrm{F}-\mathrm{H}}=4.9 \mathrm{~Hz}, 1 \mathrm{~F}\right),-127.9\left(\mathrm{dt},{ }^{2} J=240.6,{ }^{3} J_{\mathrm{F}-\mathrm{H}}=24.2 \mathrm{~Hz}, 1 \mathrm{~F}\right)$ ppm;

The following signals were attributed to the minor 4, 5-trans, 5, 7-trans-diastereoisomer $\mathbf{4 h}$ (assigned on the basis of $\delta$ and intensity); ${ }^{1} \mathrm{H}$ NMR (600 MHz, DMSO- $d_{6}, 373 \mathrm{~K}$ ): $\delta 4.90$ (br. s, 1H), 3.75 (br. s, 1H), 2.37-2.21 (m, 1H), 1.57 (br. dd, $\left.{ }^{2} J=13.9, J=8.0 \mathrm{~Hz}, 1 \mathrm{H}\right), 1.34-1.25$ $(\mathrm{m}, 1 \mathrm{H}), 1.04(\mathrm{~d}, J=7.1 \mathrm{~Hz}, 3 \mathrm{H}), 1.02(\mathrm{~s}, 3 \mathrm{H}), 0.55-0.48(\mathrm{~m}, 1 \mathrm{H}) \mathrm{ppm} ;{ }^{13} \mathrm{C} \mathrm{NMR}(150 \mathrm{MHz}$, DMSO- $\left.d_{6}, 373 \mathrm{~K}\right): \delta=125.0\left(\mathrm{t},{ }^{1} J_{\mathrm{C}-\mathrm{F}}=248.1 \mathrm{~Hz}\right), 74.7\left(\mathrm{t},{ }^{2} J_{\mathrm{C}-\mathrm{F}}=22.5 \mathrm{~Hz}\right), 73.6,36.8,35.0$ $\left(\mathrm{t},{ }^{2} J_{\mathrm{C}-\mathrm{F}}=22.2 \mathrm{~Hz}\right), 22.5,21.0\left(\mathrm{~d},{ }^{4} J_{\mathrm{C}-\mathrm{F}}=5.6 \mathrm{~Hz}\right), 13.4,8.5,7.9 \mathrm{ppm} ;{ }^{19} \mathrm{~F}$ NMR $(376 \mathrm{MHz}$, DMSO- $\left.d_{6}, 373 \mathrm{~K}\right): \delta=-106.7$ (br. d, $\left.{ }^{2} J=243.4 \mathrm{~Hz}, 1 \mathrm{~F}\right),-110.1\left(\mathrm{~d},{ }^{2} J=243.4 \mathrm{~Hz}, 1 \mathrm{~F}\right) \mathrm{ppm}$;

The following signals were attributed to the minor 4, 5-cis, 5, 7-cis-diastereoisomer $\mathbf{4 h}$ (assigned on the basis of $\delta$ and intensity); ${ }^{1} \mathrm{H}$ NMR (600 MHz, DMSO- $\left.d_{6}, 373 \mathrm{~K}\right): \delta 0.99$ (d, $J=6.7 \mathrm{~Hz}, 3 \mathrm{H}) \mathrm{ppm} ;{ }^{13} \mathrm{C} \mathrm{NMR}\left(150 \mathrm{MHz}, \mathrm{DMSO}-d_{6}, 373 \mathrm{~K}\right): \delta=37.4\left(\mathrm{~d},{ }^{3} J_{\mathrm{C}-\mathrm{F}}=8.0 \mathrm{~Hz}\right)$, 29.3, 12.1, 9.9, 8.2 ppm; ${ }^{19} \mathrm{~F}$ NMR (376 MHz, DMSO-d, $\left.373 \mathrm{~K}\right): \delta=-103.2\left(\mathrm{~d},{ }^{2} J=238.9\right.$ $\mathrm{Hz}, 1 \mathrm{~F}),-127.0\left(\mathrm{dt},{ }^{2} J=238.9,{ }^{3} J_{\mathrm{F}-\mathrm{H}}=26.5 \mathrm{~Hz}, 1 \mathrm{~F}\right) \mathrm{ppm} ; \bar{v} /($ neat $)=3427,2933,1465,1026$, $991 \mathrm{~cm}^{-1}$; HRMS (APCI): calcd for $\mathrm{C}_{10} \mathrm{H}_{15} \mathrm{~F}_{2} \mathrm{O}_{1}, 189.1091\left[\mathrm{M}-\mathrm{H}_{2} \mathrm{O}+\mathrm{H}\right]^{+}$, found: 189.1089; MS (EI): $m / z(\%): 168(12)[\mathrm{M}-2 \mathrm{~F}]^{+}$(major 4, 5-trans, 5, 7-cis diastereoisomer), 168 (21) $[\mathrm{M}-2 \mathrm{~F}]^{+}$(minor 4, 5-trans, 5, 7-trans diastereoisomer), $168(30)[\mathrm{M}-2 \mathrm{~F}]^{+}$(minor 4, 5-cis, 5, 7-cis diastereoisomer); $\mathrm{t}_{\mathrm{R}}(\mathrm{GC})=10.71$ minutes (major 4, 5-trans, 5, 7-cis diastereoisomer), 10.84 minutes (minor 4, 5-trans, 5, 7-trans diastereoisomer), 11.00 (minor 4, 5-cis, 5, 7-cis diastereoisomer). Diastereomerically pure 4, 5-trans, 5, 7-cis 4h: ${ }^{1} \mathrm{H}$ NMR $(600 \mathrm{MHz}$, DMSO- $\left.d_{6}, 373 \mathrm{~K}\right): \delta=4.84$ (br. d, $\left.J=5.5 \mathrm{~Hz}, 1 \mathrm{H}\right), 3.81$ (br. s, $\left.1 \mathrm{H}\right), 3.51\left(\mathrm{dt}, J_{\mathrm{H}-\mathrm{F}}=24.2,5.5\right.$, $J=5.5 \mathrm{~Hz}, 1 \mathrm{H}), 2.09-1.92(\mathrm{~m}, 1 \mathrm{H}), 1.74$ (br. t, $\left.{ }^{2} J=J=13.6 \mathrm{~Hz}, 1 \mathrm{H}\right), 1.14\left(\mathrm{~d},{ }^{5} J_{\mathrm{H}-\mathrm{F}}=2.2 \mathrm{~Hz}\right.$, $3 \mathrm{H}), 0.96(\mathrm{~d}, J=6.8 \mathrm{~Hz}, 3 \mathrm{H}), 0.90-0.83(\mathrm{~m}, 1 \mathrm{H}), 0.79\left(\mathrm{dt},{ }^{2} J=13.6,{ }^{4} J_{\mathrm{H}-\mathrm{F}}=J=4.6 \mathrm{~Hz}, 1 \mathrm{H}\right)$, $0.58\left(\mathrm{ddd},{ }^{2} J=9.3, J=5.7,3.8 \mathrm{~Hz}, 1 \mathrm{H}\right), 0.21-0.13(\mathrm{~m}, 1 \mathrm{H}), 0.13-0.03(\mathrm{~m}, 1 \mathrm{H}) \mathrm{ppm} ;{ }^{13} \mathrm{C}$ 
NMR $\left(150 \mathrm{MHz}, \mathrm{DMSO}-d_{6}, 373 \mathrm{~K}\right): \delta=122.7\left(\mathrm{dd},{ }^{1} J_{\mathrm{C}-\mathrm{F}}=249.8,244.8 \mathrm{~Hz}\right), 76.5\left(\mathrm{t},{ }^{2} J_{\mathrm{C}-\mathrm{F}}=\right.$ 19.4 Hz), $71.2\left(\mathrm{~d},{ }^{3} J_{\mathrm{C}-\mathrm{F}}=9.0 \mathrm{~Hz}\right), 36.8\left(\mathrm{~d},{ }^{3} J_{\mathrm{C}-\mathrm{F}}=9.0 \mathrm{~Hz}\right), 35.7\left(\mathrm{t},{ }^{2} J_{\mathrm{C}-\mathrm{F}}=21.9 \mathrm{~Hz}\right), 24.5,19.0$ $\left(\mathrm{d},{ }^{4} J_{\mathrm{C}-\mathrm{F}}=6.1 \mathrm{~Hz}\right), 10.8\left(\mathrm{dd},{ }^{3} J_{\mathrm{C}-\mathrm{F}}=5.8,2.5 \mathrm{~Hz}\right), 7.4,5.5 \mathrm{ppm} ;{ }^{19} \mathrm{~F}$ NMR $(376 \mathrm{MHz}, \mathrm{DMSO}-$ $\left.d_{6}, 373 \mathrm{~K}\right): \delta=-104.7$ (app. dq, $\left.{ }^{2} J=240.6,{ }^{3} J_{\mathrm{F}-\mathrm{H}}={ }^{4} J_{\mathrm{F}-\mathrm{H}}=4.9 \mathrm{~Hz}, 1 \mathrm{~F}\right),-127.9\left(\mathrm{dt},{ }^{2} J=240.6\right.$, $\left.{ }^{3} J_{\mathrm{F}-\mathrm{H}}=24.2 \mathrm{~Hz}, 1 \mathrm{~F}\right) \mathrm{ppm} ; \bar{v} /($ neat $)=3427,2938,1376,1231,1090,991 \mathrm{~cm}^{-1} ;$ HRMS (APCI): calcd for $\mathrm{C}_{10} \mathrm{H}_{20} \mathrm{~F}_{2} \mathrm{O}_{2} \mathrm{~N}_{1}, 224.1462\left[\mathrm{M}+\mathrm{NH}_{4}\right]^{+}$, found: 224.1458 ; MS (EI): $m / z$ (\%): $168(12)[\mathrm{M}-2 \mathrm{~F}]^{+} ; \mathrm{t}_{\mathrm{R}}(\mathrm{GC})=10.71$ minutes; Diastereomerically pure 4, 5-trans, 5, 7-trans $\mathbf{4 h}$ : m.p. $=66-68{ }^{\circ} \mathrm{C}$ (recrystallized from chloroform/pentane as a colourless plate); ${ }^{1} \mathrm{H}$ NMR (600 MHz, DMSO- $\left.d_{6}, 373 \mathrm{~K}\right): \delta 4.92$ (br. d, $\left.J=5.8 \mathrm{~Hz}, 1 \mathrm{H}\right), 3.77$ (br. s, $\left.1 \mathrm{H}\right), 3.51\left(\mathrm{dt}, J_{\mathrm{H}-\mathrm{F}}=15.2\right.$, $5.9, J=5.9 \mathrm{~Hz}, 1 \mathrm{H}), 2.34-2.22(\mathrm{~m}, 1 \mathrm{H}), 1.56$ (br. dd, $\left.{ }^{2} J=13.9, J=8.0 \mathrm{~Hz}, 1 \mathrm{H}\right), 1.29$ (br. d, , $\left.{ }^{2} J=13.9 \mathrm{~Hz}, 1 \mathrm{H}\right), 1.04(\mathrm{~d}, J=7.2 \mathrm{~Hz}, 3 \mathrm{H}), 1.01$ (s, 3H), 0.76 (br. dt, ${ }^{2} J=9.1, J=4.1 \mathrm{~Hz}$, $1 \mathrm{H}), 0.54-0.48(\mathrm{~m}, 1 \mathrm{H}), 0.17\left(\mathrm{ddd},{ }^{2} J=9.1, J=5.5,4.3 \mathrm{~Hz}, 1 \mathrm{H}\right), 0.13\left(\mathrm{ddd},{ }^{2} J=9.2, J=5.2\right.$, $3.6 \mathrm{~Hz}, 1 \mathrm{H}) \mathrm{ppm} ;{ }^{13} \mathrm{C} \mathrm{NMR}\left(150 \mathrm{MHz}, \mathrm{DMSO}-d_{6}, 373 \mathrm{~K}\right): \delta=124.0\left(\mathrm{t},{ }^{1} J_{\mathrm{C}-\mathrm{F}}=248.5 \mathrm{~Hz}\right)$, $73.6\left(\mathrm{t},{ }^{2} J_{\mathrm{C}-\mathrm{F}}=22.3 \mathrm{~Hz}\right), 72.5,35.8\left(\mathrm{t},{ }^{3} J_{\mathrm{C}-\mathrm{F}}=3.9 \mathrm{~Hz}\right), 33.9\left(\mathrm{t},{ }^{2} J_{\mathrm{C}-\mathrm{F}}=21.9 \mathrm{~Hz}\right), 21.4,20.0(\mathrm{~d}$, $\left.{ }^{4} J_{\mathrm{C}-\mathrm{F}}=4.0 \mathrm{~Hz}\right), 12.4\left(\mathrm{t},{ }^{3} J_{\mathrm{C}-\mathrm{F}}=5.1 \mathrm{~Hz}\right), 7.4,6.9 \mathrm{ppm} ;{ }^{19} \mathrm{~F}$ NMR $\left(376 \mathrm{MHz}, \mathrm{DMSO}-d_{6}, 373\right.$ $\mathrm{K}): \delta=-106.7$ (br. d, $\left.{ }^{2} J=243.4 \mathrm{~Hz}, 1 \mathrm{~F}\right),-110.1\left(\mathrm{~d},{ }^{2} J=243.4 \mathrm{~Hz}, 1 \mathrm{~F}\right) \mathrm{ppm}\left(\right.$ the ${ }^{19} \mathrm{~F}-{ }^{1} \mathrm{H}$ splitting was not resolved in the ${ }^{19} \mathrm{~F}$ NMR spectrum); $\bar{v} /($ neat $)=3382,2931,1380,1023,824$ $\mathrm{cm}^{-1}$; HRMS (APCI): calcd for $\mathrm{C}_{10} \mathrm{H}_{15} \mathrm{~F}_{2} \mathrm{O}_{1}, 189.1091\left[\mathrm{M}-\mathrm{H}_{2} \mathrm{O}+\mathrm{H}\right]^{+}$, found: 189.1087 ; MS (EI): $m / z(\%): 168(21)[\mathrm{M}-2 \mathrm{~F}]^{+} ; \mathrm{t}_{\mathrm{R}}(\mathrm{GC})=10.84$ minutes.

$\left(6 R^{*}, 7 S^{*}, 9 S^{*}\right)-8,8-D i f l u o r o-6,9-d i m e t h y l s p i r o[4.5]$ decane-6,7-diol (6, 7-trans, 7, 9trans-4i) and (6S*,7S*,9S*)-8,8-difluoro-6,9-dimethylspiro[4.5]decane-6,7-diol (6, 7-cis, 7, 9-trans-4i). Prepared according to general procedure B from 3i $(0.339 \mathrm{~g}, 1.06 \mathrm{mmol})$ with 1,3-bis(2,6-diisopropylphenyl-imidazol-2-ylidene)gold(I) chloride (5 mol \%, $0.033 \mathrm{~g}$ ) and silver hexafluoroantimonate $(\mathrm{V})(5 \mathrm{~mol} \%, 0.018 \mathrm{~g})$ in dichloromethane $(5.4 \mathrm{~mL})$ and 
methanol $(0.6 \mathrm{~mL})$. After stirring for 2 hours at $40{ }^{\circ} \mathrm{C}$ the reaction was allowed to cool to room temperature and reduced with tetrabutylammonium borohydride $(0.273 \mathrm{~g}, 1.06 \mathrm{mmol})$. The usual work up afforded a viscous pale yellow oil $(0.395 \mathrm{~g})$. The crude material was purified by flash column chromatography (40 g silica, $6 \%$ acetone in dichloromethane) to afford a mixture of 6, 7-trans, 7, 9-trans-4i, and 6, 7-cis, 7, 9-trans-4i (0.168 g). The material was further purified by flash column chromatography $(40 \mathrm{~g}$ silica, $6 \%$ acetone in dichloromethane) to afford an inseparable mixture of 6, 7-trans, 7, 9-trans-4i, and 6, 7-cis, 7, 9-trans-4i (0.108 g, $44 \%, 3.8: 1) . \mathrm{R}_{\mathrm{f}}=0.7$ (8 \% acetone in dichloromethane); The following signals were attributed to both the minor 6, 7-cis, 7, 9-trans-diastereoisomer $\mathbf{4 i}$ and major 6, 7-trans, 7, 9-trans-diastereoisomer $4 \mathbf{i}{ }^{1} \mathrm{H}$ NMR (400 MHz, $\left.\mathrm{CDCl}_{3}\right): \delta=2.48-2.13(\mathrm{~m}$, including 2.23 (br.s, 1H), 1H), 2.04-1.38 (envelope, 13H), 1.37-1.22 (m, including 1.33 (s, $3 \mathrm{H}), 6 \mathrm{H}) \mathrm{ppm}$; The following signals were attributed to the major 6, 7-trans, 7, 9-transdiastereoisomer $4 \mathbf{i}$ (assigned on the basis of $\delta$ and intensity); ${ }^{1} \mathrm{H}$ NMR (400 $\left.\mathrm{MHz}, \mathrm{CDCl}_{3}\right)$ : $\delta$ $=3.64\left(\mathrm{t}, J_{\mathrm{H}-\mathrm{F}}=5.8 \mathrm{~Hz}, 1 \mathrm{H}\right), 2.23($ br. s, $1 \mathrm{H}), 1.33(\mathrm{~s}, 3 \mathrm{H}), 1.07(\mathrm{~d}, J=6.9 \mathrm{~Hz}, 3 \mathrm{H}) \mathrm{ppm} ;{ }^{13} \mathrm{C}$ NMR (100 MHz, MeOD): $\delta=127.1\left(\mathrm{t},{ }^{1} J_{\mathrm{C}-\mathrm{F}}=247.7 \mathrm{~Hz}\right), 79.8\left(\mathrm{~d},{ }^{3} J_{\mathrm{C}-\mathrm{F}}=5.3 \mathrm{~Hz}\right), 79.2(\mathrm{dd}$, $\left.{ }^{2} J_{\mathrm{C}-\mathrm{F}}=27.8,20.7 \mathrm{~Hz}\right), 52.0,43.2\left(\mathrm{~d},{ }^{3} J_{\mathrm{C}-\mathrm{F}}=8.0 \mathrm{~Hz}\right), \quad 38.8,36.8,33.5\left(\mathrm{t},{ }^{2} J_{\mathrm{C}-\mathrm{F}}=21.5 \mathrm{~Hz}\right)$, 29.7, 27.4, 24.4, $14.3\left(\mathrm{~d},{ }^{3} J_{\mathrm{C}-\mathrm{F}}=4.3 \mathrm{~Hz}\right) \mathrm{ppm} ;{ }^{19} \mathrm{~F}$ NMR $\left(376 \mathrm{MHz}, \mathrm{CDCl}_{3}\right): \delta=-107.6\left(\mathrm{~d},{ }^{2} J\right.$ $=253.2 \mathrm{~Hz}, 1 \mathrm{~F}),-116.2\left(\mathrm{dd},{ }^{2} J=253.2,{ }^{3} J_{\mathrm{F}-\mathrm{H}}=28.7 \mathrm{~Hz}, 1 \mathrm{~F}\right) \mathrm{ppm}\left(\right.$ the ${ }^{19} \mathrm{~F}-{ }^{1} \mathrm{H}$ splitting was not resolved in the ${ }^{19} \mathrm{~F}$ NMR spectrum); The following signals were attributed to the minor 6 , 7-cis, 7, 9-trans-diastereoisomer $4 \mathbf{i}$ (assigned on the basis of $\delta$ and intensity); ${ }^{1} \mathrm{H}$ NMR (400 $\mathrm{MHz}_{\mathrm{CDCl}}$ ): $\delta=3.67\left(\mathrm{t}, J_{\mathrm{H}-\mathrm{F}}=6.6 \mathrm{~Hz}, 1 \mathrm{H}\right), 2.69$ (br. s, $\left.1 \mathrm{H}\right), 2.65$ (br. s, $\left.1 \mathrm{H}\right) 1.26\left(\mathrm{~d},{ }^{5} J_{\mathrm{H}-\mathrm{F}}=\right.$ $4.1 \mathrm{~Hz}, 3 \mathrm{H}), 1.05(\mathrm{~d}, J=6.3 \mathrm{~Hz}, 3 \mathrm{H}) \mathrm{ppm} ;{ }^{13} \mathrm{C} \mathrm{NMR}(100 \mathrm{MHz}, \mathrm{MeOD}): \delta=126.9\left(\mathrm{dd},{ }^{1} J_{\mathrm{C}-\mathrm{F}}\right.$ $=248.7,243.0 \mathrm{~Hz}), 80.5\left(\mathrm{dd},{ }^{2} J_{\mathrm{C}-\mathrm{F}}=32.9,20.4 \mathrm{~Hz}\right), 76.7\left(\mathrm{~d},{ }^{3} J_{\mathrm{C}-\mathrm{F}}=6.9 \mathrm{~Hz}\right), 52.9,41.4(\mathrm{~d}$, $\left.{ }^{3} J_{\mathrm{C}-\mathrm{F}}=8.7 \mathrm{~Hz}\right), 38.2,33.6,33.0\left(\mathrm{t},{ }^{2} J_{\mathrm{C}-\mathrm{F}}=21.8 \mathrm{~Hz}\right), 28.0,25.4,25.3\left(\mathrm{~d},{ }^{4} J_{\mathrm{C}-\mathrm{F}}=8.4 \mathrm{~Hz}\right), 13.9$ $\left(\mathrm{d},{ }^{3} J_{\mathrm{C}-\mathrm{F}}=5.4 \mathrm{~Hz}\right) \mathrm{ppm} ;{ }^{19} \mathrm{~F}$ NMR $\left(376 \mathrm{MHz}, \mathrm{CDCl}_{3}\right): \delta-105.9\left(\mathrm{dq},{ }^{2} J=253.7,{ }^{3} J_{\mathrm{F}-\mathrm{H}}={ }^{4} J_{\mathrm{F}-\mathrm{H}}=\right.$ 
$5.4 \mathrm{~Hz}, 1 \mathrm{~F}),-117.5\left(\mathrm{dd},{ }^{2} J=253.7,{ }^{3} J_{\mathrm{F}-\mathrm{H}}=28.9 \mathrm{~Hz}, 1 \mathrm{~F}\right) \mathrm{ppm}$ ( the $6.6 \mathrm{~Hz}{ }^{19} \mathrm{~F}-{ }^{1} \mathrm{H}$ splitting was not resolved in the ${ }^{19} \mathrm{~F}$ NMR spectrum); $\bar{v} /($ neat $)=3600,3331,2947,1454,1383,1086,978$ $\mathrm{cm}^{-1}$; HRMS (APCI): calcd for $\mathrm{C}_{12} \mathrm{H}_{22} \mathrm{~F}_{2} \mathrm{O}_{2} \mathrm{~N}_{1}, 252.1770$ [M-H] $]^{+}$, found: 252.1769; MS (EI): $m / z(\%): 219(1)\left[\mathrm{M}^{-\mathrm{CH}_{3}}\right]^{+} ; \mathrm{t}_{\mathrm{R}}(\mathrm{GC})=12.17$ minutes;* elemental analysis calcd $(\%)$ for $\mathrm{C}_{12} \mathrm{H}_{20} \mathrm{~F}_{2} \mathrm{O}_{2}$ : C, 61.52; H, 8.60; found: $\mathrm{C}, 61.76 ; \mathrm{H}, 8.64$. This analysis was obtained for the amorphous solid obtained following chromatography so no melting point was recorded. * the individual diastereoisomers appeared as one peak by GC-MS.

Cis-methyl (3S*,4aR*)-3-methoxy-3-methyl-4,4a,5,6,7,8-hexahydro-3H-isochromene1-carboxylate (3, 4a-cis-5) and trans-methyl (3R*,4aR*)-3-methoxy-3-methyl-4,4a,5,6,7,8hexahydro-3H-isochromene-1-carboxylate (3, 4a-trans-5). Prepared according to general procedure B from trans-3j and cis-3j $(0.253 \mathrm{~g}, 0.83 \mathrm{mmol})$ with $1,3-b i s(2,6-$ diisopropylphenyl-imidazol-2-ylidene)gold(I) chloride (5 mol \%, $0.026 \mathrm{~g})$ and silver hexafluoroantimonate $(\mathrm{V})(5 \mathrm{~mol} \%, 0.014 \mathrm{~g})$ in dichloromethane $(4.5 \mathrm{~mL})$ and methanol $(0.5$ $\mathrm{mL}$ ). After stirring for 15 minutes at $40{ }^{\circ} \mathrm{C}$ the reaction was allowed to cool to room temperature. The solvent was removed under reduced pressure and the residue taken up in ethyl acetate $(20 \mathrm{~mL})$. The organics were washed with saturated aqueous sodium bicarbonate $(15 \mathrm{~mL})$. The aqueous layer was further extracted with ethyl acetate $(3 \times 15 \mathrm{~mL})$ and the organics combined, dried over magnesium sulphate and concentrated under reduced pressure to afford the crude product as a dark orange oil $(0.204 \mathrm{~g})$. The crude material was purified by flash column chromatography using a Thomson Single Step cartridge (12 g silica, 0-5 \% acetone in dichloromethane) to afford an inseparable mixture of 3, 4a-cis $\mathbf{5}$, and 3, 4a-trans-5 as a pale yellow oil. After storing the material in the freezer at $0{ }^{\circ} \mathrm{C}$ for one year the material solidified to afford a pale yellow solid $(0.057 \mathrm{~g}, 29 \%, 7.3: 1)$. m.p. $=40-42{ }^{\circ} \mathrm{C}$ (crystals were grown by slow evaporation from chloroform/pentane under reduced pressure as small colourless prisms); $\mathrm{R}_{\mathrm{f}}=0.54$ (10\% ethyl acetate in hexane); The following signals were 
attributed to both the minor 3, 4a-trans-diastereoisomer 5 and major 3, 4a-cis-diastereoisomer $5{ }^{1} \mathrm{H}$ NMR (400 MHz, $\left.\mathrm{CDCl}_{3}\right): \delta=3.79(\mathrm{~s}, 3 \mathrm{H}), 3.53-3.42(\mathrm{~m}, 1 \mathrm{H}), 1.97-1.61$ (envelope, $5 \mathrm{H}), 1.47(\mathrm{~s}, 3 \mathrm{H}), 1.45-1.22(\mathrm{~m}, 4 \mathrm{H}) \mathrm{ppm}$; The following signals were attributed to the major 3, 4a-cis-diastereoisomer 5 (assigned on the basis of $\delta$ and intensity); ${ }^{1} \mathrm{H}$ NMR (400 MHz, $\left.\mathrm{CDCl}_{3}\right): \delta=3.25(\mathrm{~s}, 3 \mathrm{H}), 2.35-2.22(\mathrm{~m}, 1 \mathrm{H}), 2.00\left(\mathrm{dd},{ }^{2} J=13.7,7.0 \mathrm{~Hz}, 1 \mathrm{H}\right), 1.07\left(\mathrm{dq},{ }^{2} J=J\right.$ $=12.5, J=3.6 \mathrm{~Hz}, 1 \mathrm{H}) \mathrm{ppm} ;{ }^{13} \mathrm{C} \mathrm{NMR}\left(100 \mathrm{MHz}, \mathrm{CDCl}_{3}\right): \delta=164.2,132.7,128.7,97.1$, $51.2,48.6,40.2,33.3,31.7,27.1,26.2,25.1,22.2 \mathrm{ppm}$; The following signals were attributed to the minor 3, 4a-trans-diastereoisomer 5 (assigned on the basis of $\delta$ and intensity); ${ }^{1} \mathrm{H}$ NMR $\left(400 \mathrm{MHz}, \mathrm{CDCl}_{3}\right): \delta=3.31(\mathrm{~s}, 3 \mathrm{H}) \mathrm{ppm} ;{ }^{13} \mathrm{C} \mathrm{NMR}\left(100 \mathrm{MHz}, \mathrm{CDCl}_{3}\right): \delta=164.1,132.9$, $128.5,98.3,51.2,48.4,37.6,34.6,34.4,28.1,27.3,26.0,21.8 \mathrm{ppm} ; \bar{v} /($ neat $)=2921,1719$, 1435, 1279, 1115, 1045, $881 \mathrm{~cm}^{-1}$; HRMS (APCI): calcd for $\mathrm{C}_{13} \mathrm{H}_{24} \mathrm{O}_{4} \mathrm{~N}_{1}, 258.1700$ $\left[\mathrm{M}+\mathrm{NH}_{4}\right]^{+}$, found: 258.1701; MS (EI): $\mathrm{m} / \mathrm{z}(\%): 240$ (1) $[\mathrm{M}]^{+}(3,4 \mathrm{a}-\mathrm{cis}$ and 3, 4a-trans diastereoisomers); $t_{\mathrm{R}}(\mathrm{GC})=12.19$ minutes (major 3, 4a-cis diastereoisomer), 12.26 minutes (minor 3, 4a-trans diastereoisomer).

\section{General Procedure C: Propargyl Ether Preparation. 1,1-Difluoro-2-} ([methoxyethoxy]- methoxy) 3-(Propargyloxy)hexane (6a). Propargyl ether 6a was prepared according to the procedure of Percy and co-workers. ${ }^{15}$ Propargyl bromide $(0.78 \mathrm{~mL}$ of an 80 wt $\%$ solution in toluene, $7.0 \mathrm{mmol}$ ) was added dropwise to a vigorously stirred mixture of allylic alcohol 3k (1.54g, $5.4 \mathrm{mmol})$ and tetra(n-butyl)ammonium hydrogen sulfate $(0.085 \mathrm{~g}$, $0.25 \mathrm{mmol})$ in aqueous sodium hydroxide $(4.5 \mathrm{~mL}, 50 \mathrm{wt} \%)$ at $0{ }^{\circ} \mathrm{C}$. The reaction mixture was allowed to warm to room temperature and stirred for 18 hours. The mixture was quenched with aqueous saturated ammonium chloride $(20 \mathrm{~mL})$ and transferred to a separating funnel. Water $(10 \mathrm{~mL})$ was added and the product was extracted with diethyl ether $(4 \times 50$ $\mathrm{mL})$. The combined organic extracts were dried $\left(\mathrm{MgSO}_{4}\right)$, filtered and concentrated under reduced pressure to afford the crude product as a yellow oil $(1.91 \mathrm{~g})$. The crude propargyl 
ether was purified by flash column chromatography (90 g cartridge, $20 \%$ diethyl ether in hexane) to afford $\mathbf{6 a}$ as a colourless oil $(1.23 \mathrm{~g}, 71 \%) . \mathrm{R}_{\mathrm{f}}=0.62(50 \%$ diethyl ether in hexane); ${ }^{1} \mathrm{H}$ NMR $\left(400 \mathrm{MHz}, \mathrm{CDCl}_{3}\right): \delta=5.04\left(\mathrm{~d},{ }^{2} J=6.2 \mathrm{~Hz}, 1 \mathrm{H}\right), 4.95\left(\mathrm{~d},{ }^{2} J=6.2 \mathrm{~Hz}\right.$, $1 \mathrm{H}), 4.28-4.18\left(\mathrm{~m}\right.$, including $\left.4.22\left(\mathrm{dd},{ }^{2} J=15.7,{ }^{4} J=2.2 \mathrm{~Hz}, 1 \mathrm{H}\right), 1 \mathrm{H}\right), 4.09\left(\mathrm{dd},{ }^{2} J=15.7\right.$, $\left.{ }^{4} J=2.2 \mathrm{~Hz}, 1 \mathrm{H}\right), 3.94-3.76(\mathrm{~m}, 1 \mathrm{H}), 3.59(\mathrm{t}, J=4.9 \mathrm{~Hz}, 2 \mathrm{H}), 3.41\left(\mathrm{~s}, \mathrm{OCH}_{3}, 3 \mathrm{H}\right), 3.42\left(\mathrm{t},{ }^{4} J\right.$ $=2.2 \mathrm{~Hz}, 1 \mathrm{H}), 1.86-1.61(\mathrm{~m}, 2 \mathrm{H}), 1.50-1.20(\mathrm{~m}, 8 \mathrm{H}), 0.90(\mathrm{t}, J=6.9 \mathrm{~Hz}, 3 \mathrm{H}) \mathrm{ppm}$;

${ }^{13} \mathrm{C} \mathrm{NMR}\left(100 \mathrm{MHz}, \mathrm{CDCl}_{3}\right): \delta=155.7\left(\mathrm{dd},{ }^{1} J_{\mathrm{C}-\mathrm{F}}=295.0,285.5 \mathrm{~Hz}\right), 111.4\left(\mathrm{dd},{ }^{2} J_{\mathrm{C}-\mathrm{F}}=36.3\right.$. $10.5 \mathrm{~Hz}), 96.4\left(\mathrm{t},{ }^{4} J_{\mathrm{C}-\mathrm{F}}=3.3 \mathrm{~Hz}\right), 78.9,73.7,73.6\left(\mathrm{t},{ }^{4} J_{\mathrm{C}-\mathrm{F}}=3.0 \mathrm{~Hz}\right), 71.1,67.8,58.4,54.9$, 31.1, 28.4, 24.8, 22.0, $13.5 \mathrm{ppm} ;{ }^{19} \mathrm{~F}\left(376 \mathrm{MHz}, \mathrm{CDCl}_{3}\right): \delta=-96.9\left(\mathrm{~d},{ }^{2} J=62.3 \mathrm{~Hz}, 1 \mathrm{~F}\right),-$ $109.0\left(\mathrm{~d},{ }^{2} J=62.3 \mathrm{~Hz}, 1 \mathrm{~F}\right) \mathrm{ppm} ; \bar{v} /($ neat $)=3309,2924,1747,1236,1074,955 \mathrm{~cm}^{-1}$; HRMS (ESI): calcd for $\mathrm{C}_{16} \mathrm{H}_{30} \mathrm{~F}_{2} \mathrm{O}_{4} \mathrm{~N}_{1}, 338.2137\left[\mathrm{M}+\mathrm{NH}_{4}\right]^{+}$, found: $338.2139 ; \mathrm{MS}$ (CI): $\mathrm{m} / z$ (\%): 281 (1) $\left[\mathrm{M}-\mathrm{C}_{3} \mathrm{H}_{3}\right]^{+}, 265$ (4) $\left[\mathrm{M}-\mathrm{C}_{3} \mathrm{H}_{3} \mathrm{O}\right]^{+}, 245$ (6) $\left[\mathrm{M}-\mathrm{C}_{3} \mathrm{H}_{7} \mathrm{O}_{2}\right]^{+}, 153$ (4) $\left[\mathrm{C}_{10} \mathrm{H}_{17} \mathrm{O}\right]^{+}, 89$ (78) $\left[\mathrm{C}_{4} \mathrm{H}_{9} \mathrm{O}_{2}\right]^{+}, 59(100)\left[\mathrm{C}_{3} \mathrm{H}_{7} \mathrm{O}\right]^{+} ; \mathrm{t}_{\mathrm{R}}(\mathrm{GC})=12.76$ minutes.

\section{1,1-Difluoro-2-([methoxyethoxy]- methoxy) 3-(Propargyloxy)-cyclohexane (6b).}

Prepared as for 6a from allylic alcohol 3k $(2.24 \mathrm{~g}, 8.40 \mathrm{mmol})$, propargyl bromide $(1.15 \mathrm{~mL}$ of an $80 \mathrm{wt} \%$ solution in toluene, $9.9 \mathrm{mmol})$ and tetra( $n$-butyl)ammonium hydrogen sulfate $(0.126 \mathrm{~g}, 0.34 \mathrm{mmol})$ in aqueous sodium hydroxide $(6.6 \mathrm{~mL}, 50 \mathrm{wt} \%)$ at $0{ }^{\circ} \mathrm{C}$. The crude ether (2.39 g) was purified by flash column chromatography (90 g cartridge, $15 \%$ diethyl ether in hexane) to afford $\mathbf{6 b}$ as a colourless oil $(1.72 \mathrm{~g}, 68 \%) . \mathrm{R}_{\mathrm{f}}=0.42(30 \%$ diethyl ether in hexane); ${ }^{1} \mathrm{H} \mathrm{NMR}\left(400 \mathrm{MHz}, \mathrm{CDCl}_{3}\right): \delta=5.02\left(\mathrm{~d},{ }^{2} J=6.4 \mathrm{~Hz}, 1 \mathrm{H}\right), 4.92\left(\mathrm{~d},{ }^{2} J=6.4 \mathrm{~Hz}\right.$, $1 \mathrm{H}), 4.20\left(\mathrm{dd},{ }^{2} J=15.7,{ }^{4} J=2.4 \mathrm{~Hz}, 1 \mathrm{H}\right), 4.05\left(\mathrm{dd},{ }^{2} J=15.7,{ }^{4} J=2.4 \mathrm{~Hz}, 1 \mathrm{H}\right), 3.92-3.83(\mathrm{~m}$, 2H), 3.82-3.73 (m, 1H), $3.57(\mathrm{t}, J=4.9 \mathrm{~Hz}, 2 \mathrm{H}), 3.39(\mathrm{~s}, 3 \mathrm{H}),, 2.39\left(\mathrm{t},{ }^{4} J=2.4 \mathrm{~Hz}, 1 \mathrm{H}\right), 2.10$ (br. d, $\left.{ }^{2} J=13.7 \mathrm{~Hz}, 1 \mathrm{H}\right), 1.86-1.52(\mathrm{~m}, 5 \mathrm{H}), 1.37-1.09(\mathrm{~m}, 3 \mathrm{H}), 1.07-0.82(\mathrm{~m}, 2 \mathrm{H}) \mathrm{ppm} ;{ }^{13} \mathrm{C}$ NMR $\left(100 \mathrm{MHz}, \mathrm{CDCl}_{3}\right): \delta=156.2\left(\mathrm{dd},{ }^{1} J_{\mathrm{C}-\mathrm{F}}=295.5,284.9 \mathrm{~Hz}\right), 110.5\left(\mathrm{dd},{ }^{2} J_{\mathrm{C}-\mathrm{F}}=36.3\right.$. 
$10.0 \mathrm{~Hz}), 96.4\left(\mathrm{t},{ }^{4} J_{\mathrm{C}-\mathrm{F}}=3.2 \mathrm{~Hz}\right), 79.1,78.4\left(\mathrm{t},{ }^{3} J_{\mathrm{C}-\mathrm{F}}=3.5 \mathrm{~Hz}\right), 73.6,71.1,67.8,58.5,55.2$, 38.2, 29.2, 28.2, 25.9, 25.2, $25.1 \mathrm{ppm} ;{ }^{19} \mathrm{~F}\left(376 \mathrm{MHz}, \mathrm{CDCl}_{3}\right): \quad \delta=-96.8\left(\mathrm{~d},{ }^{2} J=63.7 \mathrm{~Hz}\right.$, $1 \mathrm{~F}),-109.6\left(\mathrm{~d},{ }^{2} J=63.7 \mathrm{~Hz}, 1 \mathrm{~F}\right) \mathrm{ppm} ; \bar{v} /($ neat $)=3305,2921,1745,1232,1045,957 \mathrm{~cm}^{-1}$; HRMS (APCI): calcd for $\mathrm{C}_{16} \mathrm{H}_{28} \mathrm{~F}_{2} \mathrm{O}_{4} \mathrm{~N}_{1}, 336.1986$ [M+NH$]^{+}$, found: 336.1981 ; MS (EI): $\mathrm{m} / \mathrm{z}$ (\%): $243(3)\left[\mathrm{M}_{-} \mathrm{C}_{3} \mathrm{H}_{7} \mathrm{O}_{2}\right]^{+}, 187(25)\left[\mathrm{M}-\mathrm{C}_{7} \mathrm{H}_{15} \mathrm{O}_{2}\right]^{+}, 89(71)\left[\mathrm{C}_{4} \mathrm{H}_{9} \mathrm{O}_{2}\right]^{+}, 59(100)\left[\mathrm{C}_{3} \mathrm{H}_{7} \mathrm{O}\right]^{+} ; \mathrm{t}_{\mathrm{R}}$ $(\mathrm{GC})=13.04$ minutes

1,1-Difluoro-2-([methoxyethoxy]- methoxy) 3-(Propargyloxy)- tetrahydro-2H-pyran4-yl (6c). Prepared as for 6a from allylic alcohol 3m (1.84 g, $6.5 \mathrm{mmol})$, propargyl bromide $(1.00 \mathrm{~mL}$ of an $80 \mathrm{wt} \%$ solution in toluene, $8.5 \mathrm{mmol})$ and tetra( $n$-butyl)ammonium hydrogen sulfate $(0.107 \mathrm{~g}, 0.29 \mathrm{mmol})$ in aqueous sodium hydroxide $(5.3 \mathrm{~mL}, 50 \mathrm{wt} \%)$ at 0 ${ }^{\circ} \mathrm{C}$. The crude ether (2.22 g) was purified by flash column chromatography ( $90 \mathrm{~g}$ cartridge, $50 \%$ diethyl ether in hexane) to afford $6 \mathrm{c}$ as a pale yellow oil $(1.82 \mathrm{~g}, 88 \%) . \mathrm{R}_{\mathrm{f}}=0.32(50 \%$ diethyl ether in hexane); ${ }^{1} \mathrm{H}$ NMR $\left(400 \mathrm{MHz}, \mathrm{CDCl}_{3}\right): \delta=5.01\left(\mathrm{~d},{ }^{2} J=6.6 \mathrm{~Hz}, 1 \mathrm{H}\right), 4.93(\mathrm{~d}$, $\left.{ }^{2} J=6.6 \mathrm{~Hz}, 1 \mathrm{H}\right), 4.26-4.17(\mathrm{~m}, 1 \mathrm{H}), 4.10-4.03(\mathrm{~m}, 1 \mathrm{H}), 4.02-3.82(\mathrm{~m}, 4 \mathrm{H}), 3.82-3.73(\mathrm{~m}$, $1 \mathrm{H}), 3.56(\mathrm{t}, J=5.1 \mathrm{~Hz}, 2 \mathrm{H}), 3.42-3.32(\mathrm{~m}, 5 \mathrm{H}), 2.41\left(\mathrm{t},{ }^{4} J=2.3 \mathrm{~Hz}, 1 \mathrm{H}\right), \quad 2.04-1.90(\mathrm{~m}$, 2H), $1.55-1.45(\mathrm{~m}, 1 \mathrm{H}), 1.44-1.17(\mathrm{~m}, 2 \mathrm{H}) \mathrm{ppm} ;{ }^{13} \mathrm{C} \mathrm{NMR}\left(100 \mathrm{MHz}, \mathrm{CDCl}_{3}\right): \delta=156.3(\mathrm{dd}$, $\left.{ }^{1} J_{\mathrm{C}-\mathrm{F}}=294.9,285.3 \mathrm{~Hz}\right), 110.0\left(\mathrm{dd},{ }^{2} J_{\mathrm{C}-\mathrm{F}}=36.5,10.3 \mathrm{~Hz}\right), 96.5\left(\mathrm{t},{ }^{4} J_{\mathrm{C}-\mathrm{F}}=3.4 \mathrm{~Hz}\right), 78.8,77.8$ $\left(\mathrm{t},{ }^{3} J_{\mathrm{C}-\mathrm{F}}=3.6 \mathrm{~Hz}\right), 73.9,71.1,67.8,67.1,66.8,58.5,55.2,35.7,29.5,28.0 \mathrm{ppm} ;{ }^{19} \mathrm{~F}$ NMR $\left(376 \mathrm{MHz}, \mathrm{CDCl}_{3}\right): \delta=-95.9\left(\mathrm{~d},{ }^{2} J=61.6 \mathrm{~Hz}, 1 \mathrm{~F}\right),-108.9\left(\mathrm{~d},{ }^{2} J=61.6 \mathrm{~Hz}, 1 \mathrm{~F}\right) \mathrm{ppm} ; \bar{v} /($ neat $)$ $=3263,2915,1745,1229,1046,948 \mathrm{~cm}^{-1}$; HRMS (ESI): calcd for $\mathrm{C}_{15} \mathrm{H}_{26} \mathrm{~F}_{2} \mathrm{O}_{5} \mathrm{~N}_{1}, 338.1774$

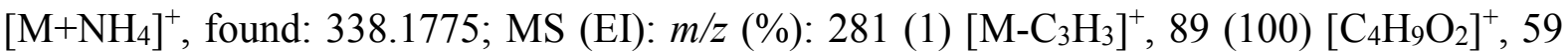
(96) $\left[\mathrm{C}_{3} \mathrm{H}_{7} \mathrm{O}\right]^{+} ; \mathrm{t}_{\mathrm{R}}(\mathrm{GC})=13.36$ minutes.

1,1-difluoro-2-([methoxyethoxy]- methoxy) 3-(Propargyloxy)- phenyl (6d). Prepared as for 6a from allylic alcohol 3n $(1.90 \mathrm{~g}, 6.9 \mathrm{mmol})$, propargyl bromide $(1.00 \mathrm{~mL}$ of an 80 wt \% solution in toluene, $9.0 \mathrm{mmol})$ and tetra $(n$-butyl)ammonium hydrogen sulfate $(0.107 \mathrm{~g}$, 
$0.31 \mathrm{mmol})$ in aqueous sodium hydroxide $(5.6 \mathrm{~mL}, 50 \mathrm{wt} \%)$ at $0{ }^{\circ} \mathrm{C}$. The crude ether $(2.14$ g) was purified by flash column chromatography (90 g cartridge, $25 \%$ diethyl ether in hexane) to afford $\mathbf{6 d}$ as a pale yellow oil $(1.71 \mathrm{~g}, 79 \%) . \mathrm{R}_{\mathrm{f}}=0.27$ (20\% diethyl ether in hexane); ${ }^{1} \mathrm{H}$ NMR (400 MHz, $\left.\mathrm{CDCl}_{3}\right): \delta=7.47-7.29(\mathrm{~m}, 5 \mathrm{H}), 5.46\left(\mathrm{t},{ }^{4} J_{\mathrm{H}-\mathrm{F}}=3.5 \mathrm{~Hz}, 1 \mathrm{H}\right)$, $4.96\left(\mathrm{~d},{ }^{2} J=6.4 \mathrm{~Hz}, 1 \mathrm{H}\right), 4.86\left(\mathrm{~d},{ }^{2} J=6.4 \mathrm{~Hz}, 1 \mathrm{H}\right), 4.28\left(\mathrm{t},{ }^{4} J=2.4 \mathrm{~Hz}, 2 \mathrm{H}\right), 3.79-3.67(\mathrm{~m}$, 1H), $3.53(\mathrm{t}, J=4.6 \mathrm{~Hz}, 2 \mathrm{H}), 3.39(\mathrm{~s}, 3 \mathrm{H}), 2.48\left(\mathrm{t},{ }^{4} J=2.4 \mathrm{~Hz}, 1 \mathrm{H}\right) \mathrm{ppm} ;{ }^{13} \mathrm{C} \mathrm{NMR}(100$ $\left.\mathrm{MHz}, \mathrm{CDCl}_{3}\right): \delta=155.6\left(\mathrm{dd},{ }^{1} J_{\mathrm{C}-\mathrm{F}}=294.3,287.7 \mathrm{~Hz}\right), 136.7,127.9,127.6,126.2,112.7(\mathrm{dd}$, $\left.{ }^{2} J_{\mathrm{C}-\mathrm{F}}=35.4,11.9 \mathrm{~Hz}\right), 96.7\left(\mathrm{t},{ }^{4} J_{\mathrm{C}-\mathrm{F}}=3.2 \mathrm{~Hz}\right), 78.5,74.5,74.4$ (app. d, $\left.{ }^{3} J_{\mathrm{C}-\mathrm{F}}=2.5 \mathrm{~Hz}\right), 71.0$, 67.8, 58.5, $55.2 \mathrm{ppm} ;{ }^{19} \mathrm{~F}$ NMR (376 MHz, $\left.\mathrm{CDCl}_{3}\right): \delta=-96.8\left(\mathrm{~d},{ }^{2} J=59.8 \mathrm{~Hz}, 1 \mathrm{~F}\right),-107.8$ $\left(\mathrm{dd},{ }^{2} J=59.8,{ }^{4} J_{\mathrm{F}-\mathrm{H}}=3.5 \mathrm{~Hz}, 1 \mathrm{~F}\right) \mathrm{ppm} ; \bar{v} /($ neat $)=3284,2920,1746,1231,1054,954 \mathrm{~cm}^{-1}$; HRMS (APCI): calcd for $\mathrm{C}_{16} \mathrm{H}_{22} \mathrm{~F}_{2} \mathrm{O}_{4} \mathrm{~N}_{1}, 330.1517$ [M+NH$]^{+}$, found: 330.1520 ; MS (EI): $\mathrm{m} / \mathrm{z}$ (\%): $145(3)\left[\mathrm{C}_{10} \mathrm{H}_{9} \mathrm{O}\right]^{+}, 89(92)\left[\mathrm{C}_{4} \mathrm{H}_{9} \mathrm{O}_{2}\right]^{+}, 59(100)\left[\mathrm{C}_{3} \mathrm{H}_{7} \mathrm{O}\right]^{+} ; \mathrm{t}_{\mathrm{R}}(\mathrm{GC})=13.42$ minutes.

1,1-Difluoro-2-([methoxyethoxy]- methoxy) 3 - (Propargyloxy)- 4(trifluoromethyl)phenyl (6e). Prepared as for 6a from allylic alcohol $30(0.813 \mathrm{~g}, 2.4 \mathrm{mmol})$, propargyl bromide $(0.35 \mathrm{~mL}$ of an $80 \mathrm{wt} \%$ solution in toluene, $3.0 \mathrm{mmol})$ and tetra $(n$ butyl)ammonium hydrogen sulfate $(0.037 \mathrm{~g}, 0.10 \mathrm{mmol})$ in aqueous sodium hydroxide (3.0 $\mathrm{mL}, 50 \mathrm{wt} \%)$ at $0{ }^{\circ} \mathrm{C}$. The crude ether $(0.917 \mathrm{~g})$ was purified by flash column chromatography (90 g cartridge, $20 \%$ diethyl ether in hexane) to afford $\mathbf{6 e}$ as a colourless oil $(0.679 \mathrm{~g}, 74 \%) . \mathrm{R}_{\mathrm{f}}=0.49$ (5\% acetone in dichloromethane); ${ }^{1} \mathrm{H}$ NMR $\left(400 \mathrm{MHz}, \mathrm{CDCl}_{3}\right): \delta$ $=7.65(\mathrm{~d}, J=8.2 \mathrm{~Hz}, 2 \mathrm{H}), 7.56(\mathrm{~d}, J=8.4 \mathrm{~Hz}, 2 \mathrm{H}), 5.51\left(\right.$ br. t $\left.,{ }^{4} J_{\mathrm{H}-\mathrm{F}}=2.7 \mathrm{~Hz}, 1 \mathrm{H}\right), 4.97(\mathrm{~d}$, $\left.{ }^{2} J=6.2 \mathrm{~Hz}, 1 \mathrm{H}\right), 4.79\left(\mathrm{~d},{ }^{2} J=6.2 \mathrm{~Hz}, 1 \mathrm{H}\right), 4.31\left(\mathrm{~d},{ }^{4} J=2.5 \mathrm{~Hz}, 2 \mathrm{H}\right), 3.75-3.69(\mathrm{~m}, 2 \mathrm{H})$, 3.55-3.49 (m, 2H), $3.39(\mathrm{~s}, 3 \mathrm{H}), 2.49\left(\mathrm{t},{ }^{4} \mathrm{~J}=2.5 \mathrm{~Hz}, 1 \mathrm{H}\right)$ ppm; ${ }^{13} \mathrm{C} \mathrm{NMR}\left(100 \mathrm{MHz}, \mathrm{CDCl}_{3}\right)$ : $\delta=155.7\left(\mathrm{dd},{ }^{1} J_{\mathrm{C}-\mathrm{F}}=294.6,287.0 \mathrm{~Hz}\right), 140.9,129.8\left(\mathrm{q},{ }^{2} J_{\mathrm{C}-\mathrm{F}}=32.8 \mathrm{~Hz}\right), 126.5,124.8\left(\mathrm{q},{ }^{3} J_{\mathrm{C}-}\right.$ $\mathrm{F}=3.5 \mathrm{~Hz}), 123.7\left(\mathrm{q},{ }^{1} J_{\mathrm{C}-\mathrm{F}}=272.7 \mathrm{~Hz}\right), 112.0\left(\mathrm{dd},{ }^{2} J_{\mathrm{C}-\mathrm{F}}=36.0,11.3 \mathrm{~Hz}\right), 96.7\left(\mathrm{t},{ }^{4} J_{\mathrm{C}-\mathrm{F}}=3.3\right.$ $\mathrm{Hz}), 78.0,74.8,73.9\left(\mathrm{t},{ }^{3} J_{\mathrm{C}-\mathrm{F}}=3.2 \mathrm{~Hz}\right), 70.9,67.9,58.5,55.4 \mathrm{ppm} ;{ }^{19} \mathrm{~F}$ NMR $(376 \mathrm{MHz}$, 
$\left.\mathrm{CDCl}_{3}\right): \delta=-62.6(\mathrm{~s}, 3 \mathrm{~F}),-95.8\left(\mathrm{~d},{ }^{2} J=58.7 \mathrm{~Hz}, 1 \mathrm{~F}\right),-107.3\left(\mathrm{dd},{ }^{2} J=58.7,{ }^{4} J_{\mathrm{F}-\mathrm{H}}=3.4 \mathrm{~Hz}\right.$ 1F) $\mathrm{ppm} ; \bar{v} /($ neat $)=3305,2921,1747,1325,1112,1067,1019 \mathrm{~cm}^{-1}$; HRMS (APCI): calcd for $\mathrm{C}_{17} \mathrm{H}_{21} \mathrm{~F}_{5} \mathrm{O}_{4} \mathrm{~N}_{1}, 398.1385$ [M+NH$]^{+}$, found: 398.1383; MS (EI): $m / z(\%): 361$ (1) [M-F] $]^{+}$, $213(4)\left[\mathrm{M}-\mathrm{C}_{7} \mathrm{H}_{10} \mathrm{~F}_{3} \mathrm{O}\right]^{+}, 89(96)\left[\mathrm{C}_{4} \mathrm{H}_{9} \mathrm{O}_{2}\right]^{+}, 59(100)\left[\mathrm{C}_{3} \mathrm{H}_{7} \mathrm{O}\right]^{+} ; \mathrm{t}_{\mathrm{R}}(\mathrm{GC})=13.00$ minutes.

1,1-Difluoro-2-([methoxyethoxy]- methoxy) 3-(Propargyloxy)- 4-(methoxy)phenyl (6f). Prepared as for 6a from allylic alcohol 3p (2.22 g, $7.3 \mathrm{mmol})$, propargyl bromide (1.06 $\mathrm{mL}$ of an $80 \mathrm{wt} \%$ solution in toluene, $9.0 \mathrm{mmol})$ and tetra( $n$-butyl)ammonium hydrogen sulfate $(0.113 \mathrm{~g}, 0.31 \mathrm{mmol})$ in aqueous sodium hydroxide $(6.0 \mathrm{~mL}, 50 \mathrm{wt} \%)$ at $0{ }^{\circ} \mathrm{C}$. The crude ether (2.73 g) was purified by flash column chromatography (90 g cartridge, $50 \%$ diethyl ether in hexane) to afford $6 \mathbf{f}$ as a pale yellow oil $(2.19 \mathrm{~g}, 88 \%) . \mathrm{R}_{\mathrm{f}}=0.39(50 \%$ diethyl ether in hexane); ${ }^{1} \mathrm{H}$ NMR (400 MHz, $\left.\mathrm{CDCl}_{3}\right): \delta=7.35(\mathrm{~d}, J=8.6 \mathrm{~Hz}, 2 \mathrm{H}), 6.90(\mathrm{~d}, J$ $=8.6 \mathrm{~Hz}, 2 \mathrm{H}), \quad 5.51-5.37\left(\mathrm{t},{ }^{4} J_{\mathrm{H}-\mathrm{F}}=3.2 \mathrm{~Hz}, 1 \mathrm{H}\right), 4.96\left(\mathrm{~d},{ }^{2} J=6.3 \mathrm{~Hz}, 1 \mathrm{H}\right), 4.76\left(\mathrm{~d},{ }^{2} J=6.3\right.$ $\mathrm{Hz}, 1 \mathrm{H}), 4.24,4.21\left(\mathrm{dABq}, J_{\mathrm{AB}}=15.9,{ }^{4} J=2.4 \mathrm{~Hz}, 2 \mathrm{H}\right), 3.81(\mathrm{~s}, 3 \mathrm{H}), 3.77-3.72(\mathrm{~m}, 2 \mathrm{H})$, $3.53(\mathrm{t}, J=4.9 \mathrm{~Hz}, 2 \mathrm{H}), 3.38(\mathrm{~s}, 3 \mathrm{H}), 2.47\left(\mathrm{t},{ }^{4} J=2.4 \mathrm{~Hz}, 1 \mathrm{H}\right) \mathrm{ppm} ;{ }^{13} \mathrm{C}$ NMR $(100 \mathrm{MHz}$, $\left.\mathrm{CDCl}_{3}\right): \delta=159.0,155.5\left(\mathrm{dd},{ }^{1} J_{\mathrm{C}-\mathrm{F}}=294.1,286.1 \mathrm{~Hz}\right), 128.7,127.5,113.3,112.8\left(\mathrm{dd},{ }^{2} J_{\mathrm{C}-\mathrm{F}}=\right.$ 34.6, 10.7 Hz), $96.7\left(\mathrm{t},{ }^{4} J_{\mathrm{C}-\mathrm{F}}=3.5 \mathrm{~Hz}\right), 78.5,74.4,74.1\left(\mathrm{t},{ }^{3} J_{\mathrm{C}-\mathrm{F}}=3.2 \mathrm{~Hz}\right), 71.0,67.8,58.5$, 55.0, $54.7 \mathrm{ppm} ;{ }^{19} \mathrm{~F}$ NMR (376 MHz, $\left.\mathrm{CDCl}_{3}\right): \delta=-97.2\left(\mathrm{~d},{ }^{2} J=60.1 \mathrm{~Hz}, 1 \mathrm{~F}\right),-107.9\left(\mathrm{~d},{ }^{2} J=\right.$ $\left.60.1,{ }^{4} J_{\mathrm{F}-\mathrm{H}}=3.2 \mathrm{~Hz}, 1 \mathrm{~F}\right) \mathrm{ppm} ; \bar{v} /($ neat $)=3283,2898,1745,1513,1247,1054,955 \mathrm{~cm}^{-1}$; HRMS (ESI): calcd for $\mathrm{C}_{17} \mathrm{H}_{24} \mathrm{~F}_{2} \mathrm{O}_{5} \mathrm{~N}_{1}, 360.1617$ [M+NH$]^{+}$, found: 360.1620 ; MS (EI): $\mathrm{m} / z$ (\%): 198 (19) $\left[\mathrm{M}-\mathrm{C}_{7} \mathrm{H}_{12} \mathrm{O}_{3}\right]^{+}, 89(76)\left[\mathrm{C}_{4} \mathrm{H}_{9} \mathrm{O}_{2}\right]^{+}, 59(100)\left[\mathrm{C}_{3} \mathrm{H}_{7} \mathrm{O}\right]^{+} ; t_{\mathrm{R}}(\mathrm{GC})=14.55$ minutes.

General Procedure D: Difluorinated Pyran Preparation. 4,4-Difluoro-2-hexyl-5methylenedihydro-2H-pyran-3(4H)-one (7a) and 3,3-dihydroxy-4,4-difluoro-2-hexyl-5methylenedihydro-2H-pyran (8a). 1,3-bis(2,6-diisopropylphenyl-imidazol-2ylidene)gold(I) chloride ( $5 \mathrm{~mol} \%, 0.031 \mathrm{~g})$ and silver hexafluoroantimonate(V) $(5 \mathrm{~mol} \%$, 
$0.017 \mathrm{~g})$ were added to a round bottom flask. 2-Methyltetrahydrofuran $(5 \mathrm{~mL})$ was added and the solution stirred at room temperature $\left(25^{\circ} \mathrm{C}\right)$. During stirring, an off-white precipitate formed. The solution was stirred at room temperature then a solution of ether $\mathbf{6 a}(0.320 \mathrm{~g}, 1$ mmol) in 2-methyltetrahydrofuran $(1 \mathrm{~mL})$ was added in a stream via syringe. The mixture was stirred for 21 hours at room temperature then concentrated under reduced pressure to afford the crude product as a viscous dark brown oil $(0.312 \mathrm{~g})$. The crude material was purified by flash column chromatography ( $40 \mathrm{~g}$ silica, $3 \%$ acetone in dichloromethane) to afford an inseparable mixture of ketone $7 \mathbf{a}$ and hydrate $\mathbf{8 a}$ as a colourless solid $(0.163 \mathrm{~g}, 65$ $\%, 1: 1)$. A small sample of crystalline hydrate 8a was prepared by recrystallization of the mixture by vapour diffusion using chloroform/pentane $(8 \mathrm{mg}, 3 \%) . \mathrm{m} . \mathrm{p} .=70-72{ }^{\circ} \mathrm{C}$ (recrystallized from chloroform/pentane as small colourless needles); $R_{\mathrm{f}}=0.57$ (10\% acetone in dichloromethane); The following signals were attributed to both ketone 7a and hydrate 8a ${ }^{1} \mathrm{H}$ NMR (400 MHz, $\mathrm{CDCl}_{3}$ ): $\delta=2.00-1.19$ (envelope, $20 \mathrm{H}$ ), $0.90(\mathrm{t}, J=7.0 \mathrm{~Hz}$, $6 \mathrm{H}) \mathrm{ppm}$; The following signals were attributed to ketone 7a (assigned on comparison of the ${ }^{1} \mathrm{H}$ NMR spectrum of crystalline hydrate 8a grown from chloroform/pentane with the ${ }^{1} \mathrm{H}$ NMR spectrum of the mixture and 2D NMR data); ${ }^{1} \mathrm{H}$ NMR (400 MHz, $\left.\mathrm{CDCl}_{3}\right): \delta=5.79$ (br. $\mathrm{d},{ }^{4} J_{\mathrm{H}-\mathrm{F}}=4.0 \mathrm{~Hz}, 1 \mathrm{H}$ ), 5.59 (app. q, ${ }^{4} J_{\mathrm{H}-\mathrm{F}}={ }^{4} J=1.4 \mathrm{~Hz}, 1 \mathrm{H}$ ), 4.54 (br. d, ${ }^{2} J=13.8 \mathrm{~Hz}, 1 \mathrm{H}$ ), 4.42 (br. d, $\left.{ }^{2} J=13.8 \mathrm{~Hz}, 1 \mathrm{H}\right), 4.17-4.10(\mathrm{~m}, 1 \mathrm{H}) \mathrm{ppm} ;{ }^{13} \mathrm{C} \mathrm{NMR}\left(100 \mathrm{MHz}, \mathrm{CDCl}_{3}\right): \delta=$ $194.7\left(\mathrm{dd},{ }^{2} J_{\mathrm{C}-\mathrm{F}}=27.9,22.9 \mathrm{~Hz}\right), 138.1\left(\mathrm{t},{ }^{2} J_{\mathrm{C}-\mathrm{F}}=18.9 \mathrm{~Hz}\right), 116.9\left(\mathrm{t},{ }^{3} J_{\mathrm{C}-\mathrm{F}}=7.5 \mathrm{~Hz}\right), 109.9$ $\left(\mathrm{dd},{ }^{1} J_{\mathrm{C}-\mathrm{F}}=261.2,246.0 \mathrm{~Hz}\right), 81.3\left(\mathrm{~d},{ }^{3} J_{\mathrm{C}-\mathrm{F}}=2.9 \mathrm{~Hz}\right), 67.8\left(\mathrm{~d},{ }^{3} J_{\mathrm{C}-\mathrm{F}}=3.3 \mathrm{~Hz}\right), 31.2,28.7$, 25.3, 24.4, 22.1, $13.5 \mathrm{ppm} ;{ }^{19} \mathrm{~F}$ NMR $\left(376 \mathrm{MHz}, \mathrm{CDCl}_{3}\right): \delta=-105.6\left(\mathrm{~d},{ }^{2} J=265.6 \mathrm{~Hz}, 1 \mathrm{~F}\right),-$ $120.0\left(\mathrm{~d},{ }^{2} \mathrm{~J}=265.6 \mathrm{~Hz}, 1 \mathrm{~F}\right) \mathrm{ppm}\left(\right.$ the ${ }^{19} \mathrm{~F}-{ }^{1} \mathrm{H}$ splittings are not resolved in the $376 \mathrm{MHz}{ }^{19} \mathrm{~F}$ NMR spectrum). The following signals were attributed to hydrate 8a (See page 162 of Results and Discussion); ${ }^{1} \mathrm{H}$ NMR (400 MHz, $\left.\mathrm{CDCl}_{3}\right): \delta=5.67\left(\mathrm{~d},{ }^{4} J_{\mathrm{H}-\mathrm{F}}=4.9 \mathrm{~Hz}, 1 \mathrm{H}\right), 5.39$ (app. q, $\left.{ }^{4} J_{\mathrm{H}-\mathrm{F}}={ }^{4} J=1.8 \mathrm{~Hz}, 1 \mathrm{H}\right), 4.32\left(\mathrm{dd},{ }^{2} J=12.9,{ }^{4} J_{\mathrm{H}-\mathrm{F}}=4.2 \mathrm{~Hz}, 1 \mathrm{H}\right), 4.17$ (br. d, ${ }^{2} J=12.9$ 
$\mathrm{Hz}, 1 \mathrm{H}), 3.65-3.50$ (m, 1H), 3.13 (br. s, $1 \mathrm{H}), 3.02$ (br. s, $1 \mathrm{H}) \mathrm{ppm} ;{ }^{13} \mathrm{C} \mathrm{NMR}(100 \mathrm{MHz}$, $\left.\mathrm{CDCl}_{3}\right): \delta=136.4\left(\mathrm{t},{ }^{2} J_{\mathrm{C}-\mathrm{F}}=20.5 \mathrm{~Hz}\right), 115.5\left(\mathrm{dd},{ }^{1} J_{\mathrm{C}-\mathrm{F}}=261.1,244.3 \mathrm{~Hz}\right), 115.2\left(\mathrm{t},{ }^{3} J_{\mathrm{C}-\mathrm{F}}=\right.$ $7.0 \mathrm{~Hz}), 92.7\left(\mathrm{dd},{ }^{2} J_{\mathrm{C}-\mathrm{F}}=27.5,20.1 \mathrm{~Hz}\right), 79.4\left(\mathrm{~d},{ }^{3} J_{\mathrm{C}-\mathrm{F}}=2.9 \mathrm{~Hz}\right), 68.7\left(\mathrm{~d},{ }^{3} J_{\mathrm{C}-\mathrm{F}}=4.4 \mathrm{~Hz}\right)$, 31.1, 28.8, 28.5, 26.0, 22.0, $13.5 \mathrm{ppm} ;{ }^{19} \mathrm{~F}$ NMR (376 MHz, $\left.\mathrm{CDCl}_{3}\right): \delta=-107.7\left(\mathrm{~d},{ }^{2} J=240.6\right.$ $\mathrm{Hz}, 1 \mathrm{~F}),-138.7\left(\mathrm{~d},{ }^{2} J=240.6 \mathrm{~Hz}, 1 \mathrm{~F}\right) \mathrm{ppm}$; (the ${ }^{19} \mathrm{~F}-{ }^{1} \mathrm{H}$ splittings are not resolved in the 376 $\mathrm{MHz}{ }^{19} \mathrm{~F}$ NMR spectrum); $\bar{v} /($ neat $)=3387,2922,1470,1242,1085,933 \mathrm{~cm}^{-1}$; HRMS (APCI): calcd for $\mathrm{C}_{12} \mathrm{H}_{19} \mathrm{~F}_{2} \mathrm{O}_{2}, 233.1353[\mathrm{M}+\mathrm{H}]^{+}$, found: $233.1352 ; *$ MS (EI): $m / z$ (\%): 232 (1) $[\mathrm{M}]^{+}, 119$ (32) $\left[\mathrm{M}-\mathrm{C}_{7} \mathrm{H}_{13} \mathrm{O}\right]^{+}, * * \mathrm{t}_{\mathrm{R}}(\mathrm{GC})=11.22$ minutes. ** *accurate mass was calculated for the ketone component of the mixture. **the mixture appeared as one peak by GC-MS, masses corresponded to that of the ketone.

2-Cyclohexyl-4,4-difluoro-5-methylenedihydro-2H-pyran-3(4H)-one (7b) and 3,3dihydroxy-2-cyclohexyl-4,4-difluoro-5-methylenedihydro-2H-pyran $(\mathbf{8 b})$. Ketone $\mathbf{7 b}$ and hydrate $\mathbf{8 b}$ were prepared according to general procedure D from propargyl ether $\mathbf{6 b}(0.318 \mathrm{~g}$, $1.00 \mathrm{mmol}$ ) with 1,3-bis(2,6-diisopropylphenyl-imidazol-2-ylidene)gold(I) chloride (5 mol $\%, \quad 0.031 \mathrm{~g})$ and silver hexafluoroantimonate(V) (5 mol \%, $0.017 \mathrm{~g})$ in 2methyltetrahydrofuran $(6.0 \mathrm{~mL})$. The crude material $(0.369 \mathrm{~g})$ was purified by flash column chromatography (40 g silica, $5 \%$ acetone in dichloromethane) to afford an inseparable mixture of ketone $\mathbf{7 b}$ and hydrate $\mathbf{8 b}$ as a colourless solid $(0.145 \mathrm{~g}, 63 \%, 7.3: 1) . \mathrm{R}_{\mathrm{f}}=0.46(5$ $\%$ acetone in dichloromethane); The following signals were attributed to both ketone $\mathbf{7 b}$ and hydrate 8b ${ }^{1} \mathrm{H}$ NMR (400 MHz, $\left.\mathrm{CDCl}_{3}\right): \delta=2.15-1.89$ (m, 1H), 1.89-1.55 (m, 5H), 1.47-1.09 $(\mathrm{m}, 5 \mathrm{H}) \mathrm{ppm}$; The following signals were attributed to ketone $\mathbf{7 b}$ (assigned on the basis of $\delta$ and intensity) ${ }^{1} \mathrm{H}$ NMR (400 MHz, $\left.\mathrm{CDCl}_{3}\right): \delta=5.82-5.74(\mathrm{~m}, 1 \mathrm{H}), 5.47$ (br. s, $\left.1 \mathrm{H}\right), 4.60$ (app. dt, $\left.{ }^{2} J=14.0,{ }^{4} J=1.4 \mathrm{~Hz}, 1 \mathrm{H}\right), 4.37$ (app. dquint, ${ }^{2} J=14.0,{ }^{4} J={ }^{4} J_{\mathrm{H}-\mathrm{F}}=1.4 \mathrm{~Hz}, 1 \mathrm{H}$ ), $3.91\left(\mathrm{dt}, J=5.0,{ }^{4} J_{\mathrm{H}-\mathrm{F}}=2.9 \mathrm{~Hz}, 1 \mathrm{H}\right) \mathrm{ppm} ;{ }^{13} \mathrm{C} \mathrm{NMR}\left(100 \mathrm{MHz}, \mathrm{CDCl}_{3}\right): \delta=195.1\left(\mathrm{t},{ }^{2} J_{\mathrm{C}-\mathrm{F}}=\right.$ $25.1 \mathrm{~Hz}), 137.9\left(\mathrm{t},{ }^{2} J_{\mathrm{C}-\mathrm{F}}=18.7 \mathrm{~Hz}\right), 116.8\left(\mathrm{t},{ }^{3} J_{\mathrm{C}-\mathrm{F}}=7.5 \mathrm{~Hz}\right), 109.6\left(\mathrm{dd},{ }^{1} J_{\mathrm{C}-\mathrm{F}}=257.0,250.5\right.$ 
$\mathrm{Hz}), 85.7,67.5,37.8,28.7,26.6,25.6 \mathrm{ppm} ;{ }^{19} \mathrm{~F}$ NMR $\left(376 \mathrm{MHz}, \mathrm{CDCl}_{3}\right): \delta=-111.7\left(\mathrm{dt},{ }^{2} J=\right.$ $\left.265.4,{ }^{4} J_{\mathrm{F}-\mathrm{H}}=2.7 \mathrm{~Hz}, 1 \mathrm{~F}\right),-120.0\left(\mathrm{dq},{ }^{2} J=265.4,{ }^{4} J_{\mathrm{F}-\mathrm{H}}=2.9 \mathrm{~Hz}, 1 \mathrm{~F}\right) \mathrm{ppm}$; The following signals were attributed to hydrate $\mathbf{8 b}$ (assigned on the basis of $\delta$ and intensity) ${ }^{1} \mathrm{H}$ NMR (400 $\left.\mathrm{MHz}, \mathrm{CDCl}_{3}\right): \delta=5.65\left(\mathrm{~d},{ }^{4} J_{\mathrm{H}-\mathrm{F}}=4.9 \mathrm{~Hz}, 1 \mathrm{H}\right), 5.37$ (app. q, $\left.{ }^{4} J_{\mathrm{H}-\mathrm{F}}={ }^{4} J=1.9 \mathrm{~Hz}, 1 \mathrm{H}\right), 4.33$ $\left(\mathrm{dd},{ }^{2} J=12.9,{ }^{4} J_{\mathrm{H}-\mathrm{F}}=4.2 \mathrm{~Hz}, 1 \mathrm{H}\right), 4.13$ (br. d, $\left.{ }^{2} J=12.9 \mathrm{~Hz}, 1 \mathrm{H}\right), 3.37-3.32$ (m, 1H), 3.24 (br. s, $1 \mathrm{H}), 3.08$ (br. s, $1 \mathrm{H}) \mathrm{ppm} ;{ }^{13} \mathrm{C} \mathrm{NMR}\left(100 \mathrm{MHz}, \mathrm{CDCl}_{3}\right): \delta=136.5\left(\mathrm{t},{ }^{2} J_{\mathrm{C}-\mathrm{F}}=20.6 \mathrm{~Hz}\right)$, $115.6\left(\mathrm{dd},{ }^{1} J_{\mathrm{C}-\mathrm{F}}=259.9,244.8 \mathrm{~Hz}\right), 115.1\left(\mathrm{t},{ }^{3} J_{\mathrm{C}-\mathrm{F}}=7.0 \mathrm{~Hz}\right), 93.9\left(\mathrm{dd},{ }^{2} J_{\mathrm{C}-\mathrm{F}}=26.6,20.1 \mathrm{~Hz}\right)$, $82.5\left(\mathrm{~d},{ }^{3} J_{\mathrm{C}-\mathrm{F}}=1.7 \mathrm{~Hz}\right), 69.0\left(\mathrm{~d},{ }^{3} J_{\mathrm{C}-\mathrm{F}}=4.4 \mathrm{~Hz}\right), 36.2,30.9,27.6,25.4 \mathrm{ppm} ;{ }^{19} \mathrm{~F}$ NMR (376 $\left.\mathrm{MHz}, \mathrm{CDCl}_{3}\right): \delta=-107.1\left(\mathrm{~d},{ }^{2} J=240.6 \mathrm{~Hz}, 1 \mathrm{~F}\right),-139.4\left(\mathrm{~d},{ }^{2} J=240.6 \mathrm{~Hz}, 1 \mathrm{~F}\right) \mathrm{ppm}\left(\right.$ the ${ }^{19} \mathrm{~F}-$ ${ }^{1} \mathrm{H}$ splittings are not resolved in the $376 \mathrm{MHz}{ }^{19} \mathrm{~F}$ NMR spectrum); $\bar{v} /($ neat $)=3404,2917$, 1452, 1217, 1091, $935 \mathrm{~cm}^{-1}$; HRMS (APCI): calcd for $\mathrm{C}_{12} \mathrm{H}_{20} \mathrm{~F}_{2} \mathrm{O}_{2} \mathrm{~N}_{1}, 248.1457\left[\mathrm{M}+\mathrm{NH}_{4}\right]^{+}$, found: 248.1459 ; $^{*} \mathrm{MS}(\mathrm{EI}): m / z(\%): 230(2)[\mathrm{M}]^{+}, 202(58)\left[\mathrm{M}-\mathrm{C}_{2} \mathrm{H}_{4}\right]^{+}, * * \mathrm{t}_{\mathrm{R}}(\mathrm{GC})=11.41$ minutes.***accurate mass was calculated for the ketone component of the mixture.**the mixture appeared as one peak by GC-MS, masses corresponded to that of the ketone.

3,3-Dihydroxy-4,4-difluoro-5-methylenehexahydro-2H,2'H-[2,4'-bipyran]

(8c).

Hydrate 8c was prepared according to general procedure D from propargyl ether $\mathbf{6 c}(0.320 \mathrm{~g}$, $1.00 \mathrm{mmol}$ ) with 1,3-bis(2,6-diisopropylphenyl-imidazol-2-ylidene)gold(I) chloride (5 mol $\%, \quad 0.031 \mathrm{~g})$ and silver hexafluoroantimonate(V) $(5 \mathrm{~mol} \%, 0.017 \mathrm{~g})$ in 2Methyltetrahydrofuran $(6.0 \mathrm{~mL})$. The crude material $(0.393 \mathrm{~g})$ was purified by flash column chromatography (40 g silica, $15 \%$ acetone in dichloromethane) to afford hydrate $8 \mathrm{c}$ as a colourless solid $(0.154 \mathrm{~g}, \quad 62 \quad \%) . \quad$ m.p. $\quad=102-104 \quad{ }^{\circ} \mathrm{C} \quad$ (recrystallized from tetrahydrofuran/pentane by vapour diffusion as a colourless needles); $R_{\mathrm{f}}=0.35$ (20\% acetone in dichloromethane); ${ }^{1} \mathrm{H}$ NMR (400 MHz, DMSO-d $): \delta=6.36(\mathrm{~s}, 1 \mathrm{H}), 6.03(\mathrm{~s}, 1 \mathrm{H}), 5.41(\mathrm{~d}$, $\left.{ }^{4} J_{\mathrm{H}-\mathrm{F}}=4.90 \mathrm{~Hz}, 1 \mathrm{H}\right), 5.34$ (br. s, $\left.1 \mathrm{H}\right), 4.30\left(\mathrm{dd},{ }^{2} J=12.9,{ }^{4} J_{\mathrm{H}-\mathrm{F}}=4.2 \mathrm{~Hz}, 1 \mathrm{H}\right), 3.94\left(\mathrm{~d},{ }^{2} J=\right.$ $12.9 \mathrm{~Hz}, 1 \mathrm{H}), 3.81\left(\mathrm{dd},{ }^{2} J=11.5, J=4.5 \mathrm{~Hz}, 2 \mathrm{H}\right), 3.29\left(\mathrm{dd},{ }^{2} J=11.5, J=2.2 \mathrm{~Hz}, 1 \mathrm{H}\right), 3.23$ 
$\left(\mathrm{dd},{ }^{2} J=11.5, J=2.2 \mathrm{~Hz}, 1 \mathrm{H}\right), 3.11\left(\mathrm{t}, J={ }^{4} J_{\mathrm{H}-\mathrm{F}}=4.0 \mathrm{~Hz}, 1 \mathrm{H}\right), 2.17-2.03(\mathrm{~m}, 1 \mathrm{H}), 1.85-1.75$ (m, $1 \mathrm{H}), 1.74-1.64(\mathrm{~m}, 1 \mathrm{H}), 1.46\left(\mathrm{qd},{ }^{2} J=J=12.1, J=4.5 \mathrm{~Hz}, 1 \mathrm{H}\right), 1.36\left(\mathrm{qd},{ }^{2} J=J=12.1\right.$, $J=4.5 \mathrm{~Hz}, 1 \mathrm{H}) \mathrm{ppm} ;{ }^{13} \mathrm{C} \mathrm{NMR}\left(150 \mathrm{MHz}, \mathrm{DMSO}-d_{6}\right): \delta=138.6\left(\mathrm{t},{ }^{2} J_{\mathrm{C}-\mathrm{F}}=19.9 \mathrm{~Hz}\right), 117.0$ $\left(\mathrm{dd},{ }^{1} J_{\mathrm{C}-\mathrm{F}}=262.8,240.9 \mathrm{~Hz}\right), 114.1\left(\mathrm{t},{ }^{3} J_{\mathrm{C}-\mathrm{F}}=7.7 \mathrm{~Hz}\right), 94.1\left(\mathrm{dd},{ }^{2} J_{\mathrm{C}-\mathrm{F}}=26.1,19.3 \mathrm{~Hz}\right), 83.0$ $\left(\mathrm{d},{ }^{3} J_{\mathrm{C}-\mathrm{F}}=2.7 \mathrm{~Hz}\right), 68.6\left(\mathrm{~d},{ }^{3} J_{\mathrm{C}-\mathrm{F}}=4.1 \mathrm{~Hz}\right), 67.7,67.6,34.0,31.7,29.0 \mathrm{ppm} ;{ }^{19} \mathrm{~F}$ NMR $(376$ MHz, DMSO- $\left.d_{6}\right): \delta=-104.6\left(\mathrm{~d},{ }^{2} J=236.8 \mathrm{~Hz}, 1 \mathrm{~F}\right),-136.0\left(\mathrm{~d},{ }^{2} J=236.8 \mathrm{~Hz}, 1 \mathrm{~F}\right) \mathrm{ppm}$; (the ${ }^{19} \mathrm{~F}-{ }^{1} \mathrm{H}$ splittings are not resolved in the $376 \mathrm{MHz}{ }^{19} \mathrm{~F}$ NMR spectrum); $\bar{v} /($ neat $)=3287,2846$, 1446, 1297, 1052, $914 \mathrm{~cm}^{-1}$; HRMS (APCI): calcd for $\mathrm{C}_{11} \mathrm{H}_{18} \mathrm{~F}_{2} \mathrm{O}_{3} \mathrm{~N}_{1}, 250.1249\left[\mathrm{M}+\mathrm{NH}_{4}\right]^{+}$,

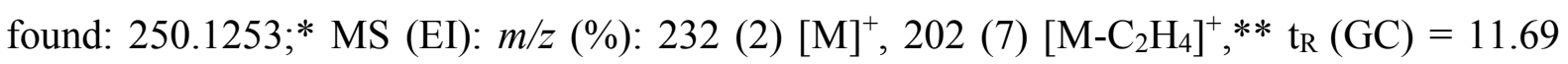
minutes. $* * *$ accurate mass was calculated for the ketone component. **the mixture appeared as one peak by GC-MS, masses corresponded to that of the ketone.

4,4-Difluoro-5-methylene-2-phenyldihydro-2H-pyran-3(4H)-one (7d) and 3,3dihydroxy-4,4-difluoro-5-methylene-2-phenyldihydro-2H-pyran (8d).

Ketone 7d and hydrate 8d were prepared according to general procedure D from propargyl ether 6d (0.312 g, 1.00 mmol) with 1,3-bis(2,6-diisopropylphenyl-imidazol-2-ylidene)gold(I) chloride (5 mol \%, $0.031 \mathrm{~g})$ and silver hexafluoroantimonate(V) (5 mol \%, $0.017 \mathrm{~g})$ in 2Methyltetrahydrofuran $(6.0 \mathrm{~mL})$. The crude material $(0.324 \mathrm{~g})$ was purified by flash column chromatography (40 g silica, $3 \%$ acetone in dichloromethane) to afford an inseparable mixture of ketone 7d and hydrate $8 \mathbf{d}$ as a colourless solid (0.126 g, 52 \%, 1:3.8). m.p. = 76$78{ }^{\circ} \mathrm{C}$ (recrystallised from ethyl acetate/hexane by vapour diffusion as a colourless plate); $\mathrm{R}_{\mathrm{f}}$ $=0.53(10 \%$ acetone in dichloromethane); The following signals were attributed to both ketone 7d and hydrate 8d ${ }^{1} \mathrm{H}$ NMR (400 $\left.\mathrm{MHz}, \mathrm{CDCl}_{3}\right): \delta=7.64-7.32(\mathrm{~m}, 5 \mathrm{H}) \mathrm{ppm}$; The following signals were attributed to ketone $7 \mathbf{d}$ (assigned on the basis of $\delta$ and intensity); ${ }^{1} \mathrm{H}$ $\operatorname{NMR}\left(400 \mathrm{MHz}, \mathrm{CDCl}_{3}\right): \delta=5.91-5.86(\mathrm{~m}, 1 \mathrm{H}), 5.58\left(\right.$ app. q, $\left.{ }^{4} J_{\mathrm{H}-\mathrm{F}}={ }^{4} J=1.5 \mathrm{~Hz}, 1 \mathrm{H}\right), 5.24$ 
$\left(\mathrm{t},{ }^{4} J_{\mathrm{H}-\mathrm{F}}=3.1 \mathrm{~Hz}, 1 \mathrm{H}\right), 4.71\left(\right.$ app. dq $\left.,{ }^{2} J=13.8,{ }^{4} J={ }^{4} J_{\mathrm{H}-\mathrm{F}}=1.1 \mathrm{~Hz}, 1 \mathrm{H}\right), 4.61\left(\right.$ br. d, ${ }^{2} J=13.8$ $\mathrm{Hz}, 1 \mathrm{H}) \mathrm{ppm} ;{ }^{13} \mathrm{C} \mathrm{NMR}\left(100 \mathrm{MHz}, \mathrm{CDCl}_{3}\right): \delta=193.0\left(\mathrm{dd},{ }^{2} J_{\mathrm{C}-\mathrm{F}}=27.3,23.4 \mathrm{~Hz}\right), 137.6(\mathrm{t}$, $\left.{ }^{2} J_{\mathrm{C}-\mathrm{F}}=19.3 \mathrm{~Hz}\right), 132.8,128.6,128.1,127.0,117.5\left(\mathrm{t},{ }^{3} J_{\mathrm{C}-\mathrm{F}}=7.4 \mathrm{~Hz}\right), 110.2\left(\mathrm{dd},{ }^{1} J_{\mathrm{C}-\mathrm{F}}=260.4\right.$, 248.7 Hz), 83.6, $67.8\left(\mathrm{~d},{ }^{3} J_{\mathrm{C}-\mathrm{F}}=2.7 \mathrm{~Hz}\right) \mathrm{ppm} ;{ }^{19} \mathrm{~F}$ NMR $\left(376 \mathrm{MHz}, \mathrm{CDCl}_{3}\right): \delta=-107.2--$ 108.0 (m, including -107.6 (app. $\left.\mathrm{d},{ }^{2} J=264.5 \mathrm{~Hz}, 1 \mathrm{~F}\right)$ ), $-116.3\left(\mathrm{~d},{ }^{2} J=264.5,1 \mathrm{~F}\right) \mathrm{ppm}$; (the ${ }^{19} \mathrm{~F}-{ }^{1} \mathrm{H}$ splittings are not resolved in the $376 \mathrm{MHz}{ }^{19} \mathrm{~F}$ NMR spectrum); The following signals were attributed to hydrate 8d (assigned on the basis of $\delta$ and intensity); ${ }^{1} \mathrm{H}$ NMR (400 MHz, $\left.\mathrm{CDCl}_{3}\right): \delta=5.73\left(\mathrm{~d},{ }^{4} J_{\mathrm{H}-\mathrm{F}}=4.9 \mathrm{~Hz}, 1 \mathrm{H}\right), 5.46\left(\right.$ app. q, $\left.{ }^{4} J_{\mathrm{H}-\mathrm{F}}={ }^{4} J=1.7 \mathrm{~Hz}, 1 \mathrm{H}\right), 4.77-4.71(\mathrm{~m}$, 1H), $4.49\left(\mathrm{dd},{ }^{2} J=13.1,{ }^{4} J_{\mathrm{H}-\mathrm{F}}=4.0 \mathrm{~Hz}, 1 \mathrm{H}\right), 4.38$ (br. d, $\left.{ }^{2} J=13.1 \mathrm{~Hz}, 1 \mathrm{H}\right), 2.88$ (br. s, $\left.2 \mathrm{H}\right)$ ppm; ${ }^{13} \mathrm{C} \mathrm{NMR}\left(100 \mathrm{MHz}, \mathrm{CDCl}_{3}\right): \delta=136.1\left(\mathrm{t},{ }^{2} J_{\mathrm{C}-\mathrm{F}}=19.9 \mathrm{~Hz}\right), 133.0,128.5,127.9,127.6$, $115.5\left(\mathrm{t},{ }^{1} J_{\mathrm{C}-\mathrm{F}}=262.8 \mathrm{~Hz}\right), 115.2\left(\mathrm{t},{ }^{3} J_{\mathrm{C}-\mathrm{F}}=7.1 \mathrm{~Hz}\right), 92.2\left(\mathrm{dd},{ }^{2} J_{\mathrm{C}-\mathrm{F}}=27.7,19.7 \mathrm{~Hz}\right), 80.5(\mathrm{~d}$, $\left.{ }^{3} J_{\mathrm{C}-\mathrm{F}}=3.2 \mathrm{~Hz}\right), 68.7\left(\mathrm{~d},{ }^{3} J_{\mathrm{C}-\mathrm{F}}=4.5 \mathrm{~Hz}\right) \mathrm{ppm} ;{ }^{19} \mathrm{~F} \mathrm{NMR}\left(376 \mathrm{MHz}, \mathrm{CDCl}_{3}\right): \delta=-106.8\left(\mathrm{~d},{ }^{2} J=\right.$ $242.3 \mathrm{~Hz}, 1 \mathrm{~F}$ ), -137.2 (app. dquint, $\left.{ }^{2} J=242.3,{ }^{4} J_{\mathrm{F}-\mathrm{H}}=2.0 \mathrm{~Hz}, 1 \mathrm{~F}\right) \mathrm{ppm} ; \bar{v} /($ neat) $=3306$, 2905, 1713, 1326, 1203, 1047, $946 \mathrm{~cm}^{-1}$; HRMS (APCI): calcd for $\mathrm{C}_{12} \mathrm{H}_{11} \mathrm{~F}_{2} \mathrm{O}_{2}, 225.0727$ $[\mathrm{M}+\mathrm{H}]^{+}$, found: $225.0723 ; * \mathrm{MS}(\mathrm{EI}): m / z(\%): 147(4)\left[\mathrm{M}-\mathrm{C}_{6} \mathrm{H}_{5}\right]^{+}, 90(100)\left[\mathrm{M}-\mathrm{C}_{9} \mathrm{H}_{9} \mathrm{O}\right]^{+}, * * t_{\mathrm{R}}$ $(\mathrm{GC})=11.82$ minutes. $* * *$ accurate mass was calculated for the ketone component of the mixture.**the mixture appeared as one peak by GC-MS, masses corresponded to that of the ketone.

\section{4,4-Difluoro-5-methylene-2-(4-(trifluoromethyl)phenyl)dihydro-2H-pyran-3(4H)-one}

(7e) and 3,3-dihydroxy-4,4-difluoro-5-methylene-2-(4-(trifluoromethyl)phenyl)dihydro-2H-

pyran $(\mathbf{8} \boldsymbol{e})$. Ketone $7 \mathbf{e}$ and hydrate $8 \mathbf{e}$ were prepared according to general procedure D from propargyl ether 6e $(0.380 \mathrm{~g}, 1.00 \mathrm{mmol})$ with 1,3-bis(2,6-diisopropylphenyl-imidazol-2ylidene)gold(I) chloride (5 mol \%, $0.031 \mathrm{~g})$ and silver hexafluoroantimonate(V) (5 mol \%, $0.017 \mathrm{~g})$ in 2-Methyltetrahydrofuran $(6.0 \mathrm{~mL})$. The crude material $(0.376 \mathrm{~g})$ was purified by flash column chromatography (40 g silica, $2 \%$ acetone in dichloromethane) to afford an 
inseparable mixture of ketone $7 \mathbf{e}$ and hydrate $8 \mathbf{e}$ as a colourless solid $(0.161 \mathrm{~g}, 52 \%, 1: 6.1)$. $\mathrm{R}_{\mathrm{f}}=0.45$ (5\% acetone in dichloromethane); The following signals were attributed to both ketone 7e and hydrate 8e ${ }^{1} \mathrm{H}$ NMR $\left(400 \mathrm{MHz}, \mathrm{CDCl}_{3}\right): \delta=7.67,7.65\left(\mathrm{ABq}, J_{\mathrm{AB}}=8.7 \mathrm{~Hz}\right.$, 4H) ppm; The following signals were attributed to ketone 7e (assigned on the basis of $\delta$ and intensity); ${ }^{1} \mathrm{H} \mathrm{NMR}\left(400 \mathrm{MHz}, \mathrm{CDCl}_{3}\right): \delta=7.49(\mathrm{~d}, J=8.4 \mathrm{~Hz}, 2 \mathrm{H}), 5.92-5.88(\mathrm{~m}, 1 \mathrm{H}), 5.61$ $\left(\mathrm{q},{ }^{4} J_{\mathrm{H}-\mathrm{F}}={ }^{4} J=1.4 \mathrm{~Hz}, 1 \mathrm{H}\right), 5.30\left(\mathrm{t},{ }^{4} J_{\mathrm{H}-\mathrm{F}}=3.2 \mathrm{~Hz}, 1 \mathrm{H}\right), 4.73\left(\mathrm{dq},{ }^{2} J=13.9,{ }^{4} J={ }^{4} J_{\mathrm{H}-\mathrm{F}}=1.2\right.$ $\mathrm{Hz}, 1 \mathrm{H}), 4.64$ (br. d, $\left.{ }^{2} J=13.9 \mathrm{~Hz}, 1 \mathrm{H}\right) \mathrm{ppm} ;{ }^{13} \mathrm{C} \mathrm{NMR}\left(100 \mathrm{MHz}, \mathrm{CDCl}_{3}\right): \delta=192.2\left(\mathrm{dd},{ }^{2} J_{\mathrm{C}-}\right.$ $\mathrm{F}=27.7,23.4 \mathrm{~Hz}), 137.3\left(\mathrm{t},{ }^{2} J_{\mathrm{C}-\mathrm{F}}=19.1 \mathrm{~Hz}\right), 136.5,130.7\left(\mathrm{q},{ }^{2} J_{\mathrm{C}-\mathrm{F}}=32.8 \mathrm{~Hz}\right), 127.2,125.0$ $\left(\mathrm{q},{ }^{3} J_{\mathrm{C}-\mathrm{F}}=3.9 \mathrm{~Hz}\right), 117.9\left(\mathrm{t},{ }^{3} J_{\mathrm{C}-\mathrm{F}}=7.5 \mathrm{~Hz}\right), 110.2\left(\mathrm{dd},{ }^{1} J_{\mathrm{C}-\mathrm{F}}=260.3,247.3 \mathrm{~Hz}\right), 82.7,68.1(\mathrm{~d}$, ${ }^{3} J_{\mathrm{C}-\mathrm{F}}=3.3 \mathrm{~Hz}$ ) ppm (the large ${ }^{1} J \mathrm{CF}_{3}$ quartet could not be observed in the $100 \mathrm{MHz}{ }^{13} \mathrm{C}$ NMR spectrum); ${ }^{19} \mathrm{~F}$ NMR $\left(376 \mathrm{MHz}, \mathrm{CDCl}_{3}\right): \delta=-62.8(\mathrm{~s}, 3 \mathrm{~F}),-107.0\left(\mathrm{~d},{ }^{2} J=265.4 \mathrm{~Hz}, 1 \mathrm{~F}\right),-$ $117.4\left(\mathrm{~d},{ }^{2} J=265.4,1 \mathrm{~F}\right) \mathrm{ppm}\left(\right.$ the ${ }^{19} \mathrm{~F}-{ }^{1} \mathrm{H}$ splittings are not resolved in the $376 \mathrm{MHz}{ }^{19} \mathrm{~F}$ NMR spectrum); The following signals were attributed to hydrate $\mathbf{8 e}$ (assigned on the basis of $\delta$ and intensity); ${ }^{1} \mathrm{H}$ NMR $\left(400 \mathrm{MHz}, \mathrm{CDCl}_{3}\right): \delta=5.76\left(\mathrm{~d},{ }^{4} J_{\mathrm{H}-\mathrm{F}}=4.8 \mathrm{~Hz}, 1 \mathrm{H}\right), 5.49$ (app. q, $\left.{ }^{4} J_{\mathrm{H}-\mathrm{F}}={ }^{4} J=1.8 \mathrm{~Hz}, 1 \mathrm{H}\right), 4.82-4.77(\mathrm{~m}, 1 \mathrm{H}), 4.51\left(\mathrm{dd},{ }^{2} J=13.1,{ }^{4} J_{\mathrm{H}-\mathrm{F}}=4.1 \mathrm{~Hz}, 1 \mathrm{H}\right), 4.38(\mathrm{br}$. d, $\left.{ }^{2} J=13.1 \mathrm{~Hz}, 1 \mathrm{H}\right), 3.09$ (br. s, 1H), 2.81 (br. s, 1H) ppm;

${ }^{13} \mathrm{C} \mathrm{NMR}\left(100 \mathrm{MHz}, \mathrm{CDCl}_{3}\right): \delta=137.2,135.7\left(\mathrm{t},{ }^{2} J_{\mathrm{C}-\mathrm{F}}=20.2 \mathrm{~Hz}\right), 130.5\left(\mathrm{q},{ }^{2} J_{\mathrm{C}-\mathrm{F}}=32.6 \mathrm{~Hz}\right)$, $128.1,124.5\left(\mathrm{q},{ }^{3} J_{\mathrm{C}-\mathrm{F}}=3.7 \mathrm{~Hz}\right), 123.8\left(\mathrm{q},{ }^{1} J_{\mathrm{C}-\mathrm{F}}=272.3 \mathrm{~Hz}\right), 115.6\left(\mathrm{t},{ }^{3} J_{\mathrm{C}-\mathrm{F}}=7.1 \mathrm{~Hz}\right), 115.3$ $\left(\mathrm{dd},{ }^{1} J_{\mathrm{C}-\mathrm{F}}=261.8,242.8 \mathrm{~Hz}\right), 92.3\left(\mathrm{dd},{ }^{2} J_{\mathrm{C}-\mathrm{F}}=28.6,20.8 \mathrm{~Hz}\right), 79.9\left(\mathrm{~d},{ }^{3} J_{\mathrm{C}-\mathrm{F}}=2.8 \mathrm{~Hz}\right), 68.7$ $\left(\mathrm{d},{ }^{3} J_{\mathrm{C}-\mathrm{F}}=4.6 \mathrm{~Hz}\right) \mathrm{ppm} ;{ }^{19} \mathrm{~F}$ NMR $\left(376 \mathrm{MHz}, \mathrm{CDCl}_{3}\right): \delta=-62.7(\mathrm{~s}, 3 \mathrm{~F}),-106.9\left(\mathrm{~d},{ }^{2} J=241.7\right.$ $\mathrm{Hz}, 1 \mathrm{~F}$ ), -137.2 (app. dquint, $\left.{ }^{2} J=241.7,{ }^{4} J_{\mathrm{F}-\mathrm{H}}=1.9 \mathrm{~Hz}, 1 \mathrm{~F}\right) \mathrm{ppm} ; \bar{v} /($ neat) $=3621,3259$, 2921, 1708, 1323, 1067, $952 \mathrm{~cm}^{-1}$; HRMS (APCI): calcd for $\mathrm{C}_{13} \mathrm{H}_{13} \mathrm{~F}_{5} \mathrm{O}_{2} \mathrm{~N}_{1}, 310.0861$ $\left[\mathrm{M}+\mathrm{NH}_{4}\right]^{+}$, found: 310.0857 ; $^{*} \mathrm{MS}(\mathrm{EI}): \mathrm{m} / z(\%): 292(1)[\mathrm{M}]^{+}, 145(63)\left[\mathrm{C}_{7} \mathrm{H}_{4} \mathrm{~F}_{3}\right]^{+}, 90(100)$ $\left[\mathrm{M}-\mathrm{C}_{10} \mathrm{H}_{8} \mathrm{~F}_{3} \mathrm{O}\right]^{+}, * * \mathrm{t}_{\mathrm{R}}(\mathrm{GC})=11.57$ minutes. $* * *$ accurate mass was calculated for the ketone 
component of the mixture.**the mixture appeared as one peak by GC-MS, masses corresponded to that of the ketone.

\section{Supporting Information}

The Supporting Information is available free of charge on the ACS Publications website.

Copies of spectroscopic data $\left({ }^{1} \mathrm{H},{ }^{13} \mathrm{C}\right.$ and $\left.{ }^{19} \mathrm{~F}\right)$ for all products and X-ray crystallographic data.

\section{Acknowledgements}

We thank the Carnegie Trust for a Carnegie-Caledonian PhD scholarship and Dr Vipulkumar Patel (GSK Flexible Discovery Unit) for additional support, the EPSRC National Mass Spectrometry Service Centre, Swansea for accurate mass measurements, Dr Alan R Kennedy for crystal structures and the EPSRC UK National Crystallography Service, Southampton for data collection on compound 8c and Craig Irving (NMR Technician, University of Strathclyde) for assistance with NMR experiments.

\section{References}

(1) (a) Müller, K.; Faeh, C.; Diederich, F. Fluorine in Pharmaceuticals: Looking Beyond Intuition. Science. 2007, 317, 1881-1886. (b) Fäh, C.; Hardegger, L. A.; Schweizer, W. B.; Meyer, S.; Bur, D.; Diederich, F. New organofluorine building blocks: inhibition of the malarial aspartic proteases plasmepsin II and IV by alicyclic $\alpha, \alpha$-difluoroketone hydrates. Org. Biomol. Chem. 2009, 7, 3947-3957. (c) Silva, A. M.; Cachau, R. E.; Sham, H. L.; Erickson, J. W. Inhibition and catalytic mechanism of HIV-1 aspartic protease. J. Mol. Biol. 1996, 255, 321-346. (d) Han, C.; Salyer, A. E.; Kim, E. H.; Jiang, X.; Jarrard, R. E.; Powers, 
M. S.; Kirchoff, A. M.; Salvador, T. K.; Chester, J. A.; Hockerman, G. H.; Colby, D. A. Evaluation of Difluoromethyl Ketones as Agonists of the $\gamma$-Aminobutyric Acid Type B (GABA $)$ Receptor. J. Med. Chem. 2013, 56, 2456-2465. (e) Hagmann, W. K. The Many Roles for Fluorine in Medicinal Chemistry. J. Med. Chem. 2008, 51, 4359-4369.

(2) Kageshima, Y.; Suzuki, C.; Oshiro, K.; Amii, H. Highly Controlled Ring-Opening of Siloxydifluorocyclopropanes: A Versatile Route to Cyclic Fluoroketones. Synlett, 2015, 6366.

(3) Kosobokov, M. D.; Levin, V. V.; Struchkova, M. I.; Dilman, A. D. Difluorohomologation of Ketones. Org. Lett. 2015, 17, 760-763.

(4) (a) Barth, F.; Yang, C. O. Cyclization of $\alpha, \alpha$-Difluoromethyl Radicals: A New Route to the Preparation of Difluorocyclopentane Derivatives. Tetrahedron. Lett. 1991, 32, 5873-5876. (b) Morikawa, T.; Kodama, Y.; Uchida, J.; Takano, M.; Washio, Y.; Taguchi, T. Cyclization reactions of $\beta, \beta$-difluoroalkyl radicals $\left(-\mathrm{CF}_{2}-\mathrm{C} \cdot\right)$ for synthesizing gem-difluorocyclic compounds. Tetrahedron. 1992, 48, 8915-8926. (c) Buttle, L. A.; Motherwell, W. B. Comparative studies on the generation and cyclisation reactions of difluoroalkyl radicals. Tetrahedron Lett. 1994, 35, 3995-3998. (d) Harrington, P. E.; Tius, M. A. Difluorocyclopentenone Synthesis. J. Org. Chem. 1999, 64, 4025-4029. (e) Yang, Y. Y.; Meng, W. D.; Qing, F. L. Synthesis of 2‘,3'-Dideoxy-6,6'-difluorocarbocyclic Nucleosides. Org. Lett. 2004, 6, 4257-4259. (f) Miles, J. A. L.; Mitchell, L.; Percy, J. M.; Singh, K.; Uneyama, E. Total Syntheses of Conformationally Locked Difluorinated Pentopyranose Analogues and a Pentopyranosyl Phosphate Mimetic. J. Org. Chem. 2007, 72, 1575-1587. (g) Anderl, T.; Audouard, C.; Miah, A.; Percy, J. M.; Rinaudo, G.; Singh, K. Syntheses of difluorinated carbasugar phosphates from trifluoroethanol. Org. Biomol. Chem. 2009, 7, 5200-5206. (h) Orr, D.; Percy, J. M.; Tuttle, T.; Kennedy, A. R.; Harrison, Z. A. Evaluating the Thermal Vinylcyclopropane Rearrangement (VCPR) as a Practical Method for the 
Synthesis of Difluorinated Cyclopentenes: Experimental and Computational Studies of Rearrangement Stereospecificity. Chem. Eur. J. 2014, 20, 14305-14316.

(5) Percy, J. M.; McCarter, A. W.; Sewell, A. L.; Sloan, N. S.; Kennedy, A. R.; Hirst, D. J. Developing the Saegusa-Ito Cyclisation for the Synthesis of Difluorinated Cyclohexenones. Chem. Eur. J. 2015, 21, 19119-19127.

(6) (a) Patel, S. T.; Percy, J. M.; Wilkes, R. D. [2,3]-Wittig Rearrangements of Difluoroallylic Ethers. A Facile Entry to Highly Functionalized Molecules Containing a $\mathrm{CF}_{2}$ Group. J. Org. Chem. 1996, 61, 166-173. (b) Griffith , G. A; Percy, J. M.; Pintat, S.; Smith, C. A.; Spencer, N.; Uneyama, E. Towards novel difluorinated sugar mimetics; syntheses and conformational analyses of highly-functionalised difluorinated cyclooctenones. Org. Biomol. Chem. 2005, 3, 2701-2712. (c) Griffith, G. A.; Hillier, I. H.; Percy, J. M.; Roig, R.; Vincent, M. A. Quantifying the Reactivity of a Remarkably Long-Lived Difluorinated Enol in Acidic Methanol via Solution Kinetics and Electronic Structure Calculations. J. Org. Chem. 2006, 71, 8250-8255.

(7) For examples of gold(I) catalyzed reactions involving enol and enol ether nucleophiles, see (a) Zhou, C.; Che, C. Highly Efficient Au(I)-Catalyzed Intramolecular Addition of $\beta$ Ketoamide to Unactivated Alkenes. J. Am. Chem. Soc. 2007, 129, 5828-5829. (b) Xiao, Y.; Liu, X.; Che, C. Efficient Gold(I)-Catalyzed Direct Intramolecular Hydroalkylation of Unactivated Alkenes with $\alpha$-Ketones. Angew. Chem. Int. Ed. 2011, 50, 4937-4941. (c) Xiao, Y.; Liu, X.; Che, C. Efficient gold(I)/silver(I)-cocatalyzed cascade intermolecular N-Michael addition/intramolecular hydroalkylation of unactivated alkenes with $\alpha$-ketones. Beilstein. J. Org. Chem. 2011, 7, 1100-1107. (d) Nevado, C.; Cárdenas, D. J.; Echavarren, A. M. Reaction of Enol Ethers with Alkynes Catalyzed by Transition Metals: 5exo-dig versus 6endodig Cyclizations via Cyclopropyl Platinum or Gold Carbene Complexes. Chem. Eur. J. 2003, $9,2627-2635$ 
(8) Patel, S. T.; Percy, J. M.; Wilkes, R. D. [3,3]-Sigmatropic Rearrangement Routes to $\beta$ Fluoro- and $\beta, \beta$-Difluoro-carbonyl Derivatives. Tetrahedron. 1995, 51, 11327-11336.

(9) Staben, S. T.; Kennedy-Smith, J. J.; Huang, D.; Corkey, B. K.; Lalonde, R. L.; Toste, F. D. Gold(I)-Catalyzed Cyclizations of Silyl Enol Ethers: Application to the Synthesis of (+)Lycopladine A. Angew. Chem. Int. Ed. 2006, 45, 5991-5994.

(10) Fructos, M. R.; Belderrain, T. R.; Frémont, P. D.; Scott, N. M.; Nolan, S. P.; DiazRequejo, M.; Pérez, P. J. A Gold Catalyst for Carbene-Transfer Reactions from Ethyl Diazoacetate. Angew. Chem. Int. Ed. 2005, 44, 5284-5288.

(11) Gatling, S. C.; Jackson, J. E. Reactivity Control via Dihydrogen Bonding: Diastereoselection in Borohydride Reductions of $\alpha$-Hydroxyketones. J. Am. Chem. Soc. 1999, $121,8655-8656$.

(12) Wigfield, D. C. Stereochemistry and mechanism of ketone reductions by hydride reagents. Tetrahedron, 1976, 35, 449-462.

(13) Lovering, F.; Bikker, J.; Humblet, C. J. Med. Chem. 2009, 52, 6752-6756.

(14) See Supporting Information.

(15) Kumar, D.; Sharma, P.; Singh, H.; Nepali, K.; Gupta, G. K.; Jain, S. K.; Ntie-Kang, F. The value of pyrans as anticancer scaffolds in medicinal chemistry. $R S C A d v . \mathbf{2 0 1 7}, 7,36977$ 36999.

(16) Guthrie, J. P. Hydration of Carbonyl Compounds, an Analysis in Terms of Multidimensional Marcus Theory. J. Am. Chem. Soc. 2000, 122, 5529-5538.

(17) Marchetti, F.; Pampaloni, G.; Repo, T. The Polymerization of Tetrahydrofuran Initiated by Niobium(V) and Tantalum(V) Halides. Eur. J. Inorg. Chem. 2008, 2107-2112.

(18) Ripin, D. H. B.; Vetelino, M. 2-Methyltetrahydrofuran as an Alternative to Dichloromethane in 2-Phase Reactions. Synlett. 2003, 2353-2353. 
(19) Coles, S. J.; Gale, P. A. Changing and challenging times for service crystallography. Chem. Sci. 2012, 3, 683-689.

(20) Sheldrick, G. M. A short history of SHELX. Acta Cryst. 2008, A64, 112-122.

(21) Altomare, A.; Cascarano, G.; Giacovazzo, C.; Guagliardi, A.; Burla, M. C.; Polidori, G.; Camalli, M. SIRPOW.92 - a program for automatic solution of crystal structures by direct methods optimized for powder data. J. Appl. Cryst. 1994, 27, 435.

(22) Duhamel, L.; Plaquevent, J. C. 4-Phenylbenzylidene benzylamine: A new and convenient reagent for the titration of solutions of lithium alkyls and metal amides. $J$. Organomet. Chem 1993, 448, 1-3.

(23) Harrison, T. J.; Kozak, J. A.; Corbella-Pané, M.; Dake, G. R. Pyrrole Synthesis Catalyzed by AgOTf or Cationic Au(I) Complexes. J. Org. Chem. 2006, 71, 4525-4529. 